\title{
Bisection auctions
}

Citation for published version (APA):

Grigorieva, E. (2007). Bisection auctions. [Doctoral Thesis, Maastricht University]. Universitaire Pers Maastricht. https://doi.org/10.26481/dis.20070328eg

Document status and date:

Published: 01/01/2007

DOI:

10.26481/dis.20070328eg

Document Version:

Publisher's PDF, also known as Version of record

\section{Please check the document version of this publication:}

- A submitted manuscript is the version of the article upon submission and before peer-review. There can be important differences between the submitted version and the official published version of record.

People interested in the research are advised to contact the author for the final version of the publication, or visit the DOI to the publisher's website.

- The final author version and the galley proof are versions of the publication after peer review.

- The final published version features the final layout of the paper including the volume, issue and page numbers.

Link to publication

\footnotetext{
General rights rights.

- You may freely distribute the URL identifying the publication in the public portal. please follow below link for the End User Agreement:

www.umlib.nl/taverne-license

Take down policy

If you believe that this document breaches copyright please contact us at:

repository@maastrichtuniversity.nl

providing details and we will investigate your claim.
}

Copyright and moral rights for the publications made accessible in the public portal are retained by the authors and/or other copyright owners and it is a condition of accessing publications that users recognise and abide by the legal requirements associated with these

- Users may download and print one copy of any publication from the public portal for the purpose of private study or research.

- You may not further distribute the material or use it for any profit-making activity or commercial gain

If the publication is distributed under the terms of Article $25 \mathrm{fa}$ of the Dutch Copyright Act, indicated by the "Taverne" license above, 
BISECTION AUCTIONS 
This book was typeset by the author using LaTeX.

Bisection auctions

(C) Elena Grigorieva, Maastricht 2007

All rights reserved. No part of this publication may be reproduced, stored in a retrieval system, or transmitted, in any form, or by any means, electronic, mechanical, photocopying, recording or otherwise, without the prior permission in writing from the author.

Published by Universitaire Pers Maastricht ISBN 978-90-5278-608-7

Printed in the Netherlands by Datawyse 


\title{
BISECTION AUCTIONS
}

\author{
Proefschrift
}

ter verkrijging van de graad van doctor aan de Universiteit Maastricht, op gezag van de Rector Magnificus, Prof. mr. G.P.M.F.Mols, volgens het besluit van het College van Decanen, in het openbaar te verdedigen op woensdag 28 maart 2007 om 16.00 uur

door

\section{Elena Grigorieva}

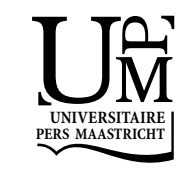




\section{Promotores:}

Prof. dr. P.J.J. Herings

Prof. dr. R.J. Müller

\section{Copromotor:}

Dr. A.J. Vermeulen

\section{Beoordelingscommissie:}

Prof. dr. H.J.M. Peters (voorzitter)

Dr. A. Predtetchinski

Prof. dr. A.J.J. Talman (Universiteit van Tilburg)

Het in dit proefschrift beschreven onderzoek werd financieel mede mogelijk gamaakt door Nederlandse Organisatie voor Wetenschappelijk Onderzoek (NWO). 


\section{Acknowledgements}

The origin of the book you hold lies in the early autumn of 2000 when Rudolf Müller offered me a position of research fellow at the International Institute of Infonomics. His interest in auctions has been so inspiring that I was also gripped by the auction fever. One year later he encouraged me to become a $\mathrm{PhD}$ student at Maastricht University. It did not take me long to make up my mind and begin a journey toward the doctorate title.

Before I even started writing this thesis, I already knew that the part where I say "thank you" would not be easy to write. The well-known "thank you" paradox states: the more persons and details you mention, the higher the probability that you forget someone. Therefore, I would like to express my one big "THANK YOU" to everyone who contributed in one way or another to the successful end of my journey and made these past few years such an enjoyable and interesting time.

However, to make it a bit more personal after all, I would like to mention some groups of people and some individuals for their very special contributions to this work. My thanks and appreciation go to my supervisors, Jean-Jacques Herings, Rudolf Müller, and Dries Vermeulen, who have been dedicated to my research and supported me in a very friendly manner. Without the stimulating environment they created, this thesis would have never been completed. I would like to thank Martin Strobel for his collaboration on the experimental part of this thesis. My colleagues from Maastricht University have provided me with a very pleasant working atmosphere, for which I am truly grateful. I would like to thank the members of the committees who have generously given their time and expertise to read my work and comment on it.

My special thanks to my friends; though they could not help me with the research itself, they gave me the much needed distraction from it. My biggest gratitude 


\section{ACKNOWLEDGMENTS}

goes to my parents and parents-in-law for their unconditional love and immense confidence in me. My warmest thanks are to my husband, Alexander, for standing by me and sharing with me the hardest, but most important and pleasant job of raising our children, Jouri and Sofia, who are my pride and joy.

Elena Grigorieva

Maastricht, January 2007 


\section{Contents}

1 Introduction $\quad 11$

I Bisection auctions in the discrete setting $\quad 19$

2 The bisection auction $\quad 21$

2.1 Introduction . . . . . . . . . . . . . . . . . . 21

2.2 The rules of the bisection auction . . . . . . . . . . . . . . 24

2.3 Formal representation of the auction . . . . . . . . . . . 26

2.4 Playing the game . . . . . . . . . . . . . . . . . . 29

2.4.1 Equivalence of strategies and threshold strategies . . . . . . . 29

2.4.2 Playing the game with threshold strategies . . . . . . . . . 32

2.4.3 Some remarks on generalizations of the bisection auction . . 34

2.5 Conclusion ........................... 35

3 Bounds on communication performance $\quad 37$

3.1 Introduction . . . . . . . . . . . . . . . . . 37

3.2 Communication performance of the bisection auction . . . . . . . . 38

3.2.1 Bounds on data transmission in the bisection auction . . . . 39

3.2.2 Comparison with the English auction . . . . . . . . . . . . 43

3.2.3 Comparison with the Vickrey auction ........... 45

3.3 Conclusion . . . . . . . . . . . . . . . . . . 46

4 Optimal communication performance $\quad 47$

4.1 Introduction . . . . . . . . . . . . . . . . . 47

4.2 Vickrey auctions as algorithms . . . . . . . . . . . . . 49

4.3 A criterium for communication performance of algorithms . . . . . . 52 
4.4 Optimal communication performance of the bisection algorithm in case of two bidders . . . . . . . . . . . . . . . . . . 5 55

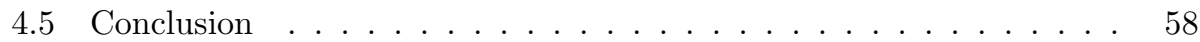

5 An experimental investigation of bidding behavior in the bisection auction

5.1 Introduction . . . . . . . . . . . . . . . . . . . . . . 59

5.2 Experimental design and procedure . . . . . . . . . . . . . . 61

5.3 Experimental results . . . . . . . . . . . . . . . . . . 63

5.3 .1 Bidding behavior . . . . . . . . . . . . . . 63

5.3 .2 Learning process . . . . . . . . . . . . . . . . 66

5.3.3 Revenue . . . . . . . . . . . . . . . . . . . 69

5.3 .4 Efficiency ...................... 71

5.3.5 Post-experimental questionnaire . . . . . . . . . . . . . 72

5.4 Conclusion . . . . . . . . . . . . . . . . . 75

5.5 Appendix 1: Subjects instructions . . . . . . . . . . . 80

5.6 Appendix 2: Post-experimental questionnaire . . . . . . . . . . 87

5.7 Appendix 3: Tables with data used for the conducted tests . . . . . 90

II Bisection auctions in the continuous setting 97

6 Inefficiency of equilibria in query auctions with continuous valuations $\quad 99$

6.1 Introduction . . . . . . . . . . . . . . . . . 99

6.2 Preliminaries . . . . . . . . . . . . . . . . . . 101

6.3 The theorem of Green and Laffont . . . . . . . . . . . . . . . . . . 103

6.4 Efficient query equilibria are almost always infinite . . . . . . . . . . 105

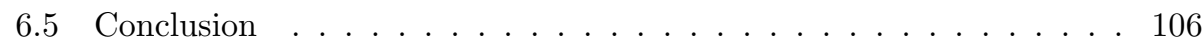

7 General bisection auctions $\quad 107$

7.1 Introduction . . . . . . . . . . . . . . . . 107

7.2 Formal representation of a general bisection auction . . . . . . . . 109

7.3 Ex post equilibrium of a general bisection auction . . . . . . . . . . . 112

7.4 Finite running time . . . . . . . . . . . . . . . . 116

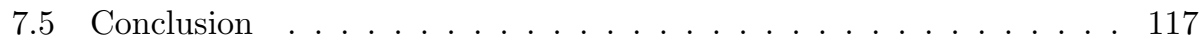

8 The family of $c$-bisection auctions: efficiency and running time 119

8.1 Introduction . . . . . . . . . . . . . . . . . 119

8.2 The $c$-bisection auction . . . . . . . . . . . . . 121 
8.3 Running time of the $c$-bisection auction . . . . . . . . . . . 125

8.3.1 The expected number of rounds . . . . . . . . . . . . 125

8.3.2 The expected number of queries . . . . . . . . . . . . 129

8.4 Efficiency of the $c$-bisection auction . . . . . . . . . . . . 132

8.4.1 The direct $c$-bisection auction . . . . . . . . . . . . . . 132

8.4.2 The probability of inefficient allocation . . . . . . . . . . . 135

8.4.3 The expected loss of welfare . . . . . . . . . . . . . . . 137

8.5 Conclusion . . . . . . . . . . . . . . . . . . . 140

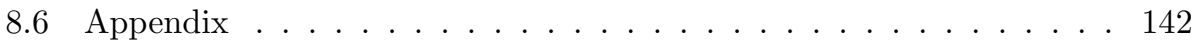

$\begin{array}{ll}\text { Bibliography } & 145\end{array}$

$\begin{array}{ll}\text { Subject Index } & 151\end{array}$

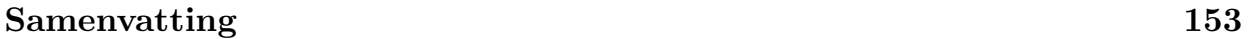

$\begin{array}{ll}\text { Curriculum Vitae } & 157\end{array}$ 



\section{Chapter 1}

\section{Introduction}

A central issue in economics is allocation of goods. One of the best ways to allocate goods is to sell them using free market mechanisms and an auction such a mechanism. Traditionally, art objects, collectibles and antiques have been sold by means of auctions. Also, the rights to use natural resources from public property such as off-shore oil lease, have often been auctioned off. In the past two decades auctions were extensively used to facilitate the transfer of assets from public to private hands, such as industrial enterprizes in Eastern Europe and the former Soviet Union, and transportation systems in Britain and Scandinavia. Recently governments of many countries have made use of auctions to sell UMTS spectrum frequencies for mobile communication. In the last few years, due to modern information technologies both the range and the total value of goods sold by auctions has grown enormously. The Internet is used nowadays to conduct hundreds of thousands of different online auctions each day with bidders worldwide.

The increasing popularity of auctions has raised a lot of questions on the appropriate design of auctions for a particular situation and captured attention of many researchers. Auctions have grown in interest within different scientific communities especially among economists, game theorists and computer scientists.

Auctions are basically about allocating and pricing scarce goods ${ }^{1}$ in a setting of uncertainty. On the first place, there is uncertainty on the seller side in the sense he is unsure of the price he can get for the object. In fact nearly any good whose value varies enough to preclude direct and absolute pricing, is a candidate to be auctioned off. A common feature of all auctions is that they elicit information, in

\footnotetext{
${ }^{1}$ In this book the words good, item and object are used interchangeably to refer to a commodity being sold. Also the words buyer, bidder, agent and player are used interchangeably to refer to a person who participates in an auction.
} 
the form of bids, from potential buyers regarding their valuations for the good(s) being sold, typically in form of the maximum amount each buyer is willing to pay. The outcome of the auction - who gets the object and at what price - is determined on the basis of the received information on bidders' valuations.

In its classical form one seller, the auctioneer, wants to find a buyer among a group of bidders who is willing to pay the highest price. Auctions may not only be used to sell goods but also to purchase them. In procurement or reverse auctions bidders compete for the right to sell their product or service. In this case the auctioneer, being a buyer, wants to find a seller among a group of bidders who suggest the lowest price.

There are different ways to classify auctions. There are for example open auctions and sealed-bid auctions, auctions where the price rises and auctions where the price drops. This results in four basic auction formats are widely used and analyzed: English, Dutch, sealed bid first price and sealed bid second price auctions. In describing their rules we focus for simplicity on the sale of a single object.

In the English auction, the price is successively raised until only one bidder remains, and that bidder wins the object at the final price. The auction can run by having the seller announce prices, or by having the bidders call out prices themselves. Antiques and artworks are commonly sold using versions of the English auction. Now over $80 \%$ of all online auctions implement this format (Lucking-Reiley [39]).

The Dutch auction (named after its best known example, the flower auctions in the Netherlands) is a descending auction where the auctioneer starts at a very high price, and then lowers the price continuously. The first bidder who calls out that he agrees to accept the current price wins the object at that price. In Dutch auctions the price is reduced rapidly so as to make the auction efficient in terms of real time, thus the Dutch auction is an attractive format for selling perishable goods.

In the sealed bid first price auction each bidder independently submits a single bid, without seeing others' bids, and the object is sold to the bidder who makes the highest bid. The winner pays his own bid. This auction format is used in auctioning mineral rights in government-owned land and sometimes in the sale of real estate. In its reverse version it is the typical auction used for procurement in the public sector.

In the sealed bid second price auction the item is awarded to the highest bidder at a price equal to the second-highest bid. This format is also known as the Vickrey auction, named after William Vickrey who wrote the seminal paper on auctions [59]. This format is implemented in some auctions on the Internet (Lucking-Reiley 
$[39,40])$ but it is much less used than the other standard formats.

The appropriate choice among auction formats depends a lot on the particular setting with respect to bidders' preferences. In the private-value setting the value of the good depends only on the bidder's own preferences. A bidder is not certain about the values of others, but knowledge of other bidders' values would not affect his own valuation. The private value assumption is natural in auctions for nondurable consumer goods. In the interdependent (or correlated) value setting values are dependent partly on own preferences and may be affected by preferences of other bidders. For example, a bidder's value for a painting may depend mostly on how much he likes it but also somewhat on how much others like it, because this affects the resale value and/or the prestige of owning it. In the common value setting the actual value is the same for everyone, but bidders have different private information about what that value actually is. For example, the value of an oil-lease depends on how much oil is under the ground and bidders may have access to different geological information about that amount. In this case a bidder would change his estimate of the value if he learned another bidder's information, in contrast to the private value case.

When several objects are sold at the same time, bidder's value for a bundle is often not simply additive. For example, when many identical goods are for sale a buyer might have decreasing or increasing marginal values (that is, the value of an additional unit decreases or, respectively, increases with the number of units already obtained), or might even have a positive value only for a single unit. When heterogeneous goods are for sale the buyers' valuation on bundles of goods might exhibit complementarity (that is, a bundle is worth more than the sum of its parts) or substitutability (that is, a bundle is worth less than the sum of its parts). Auction design addresses these issues in the form of combinatorial auctions, in which buyers are allowed to submit bids on bundles of goods.

When it comes to the comparison of different auction formats, various performance measures can be used (see e.g. Krishna [34]). From the perspective of the seller, it is natural to use revenue as a criterion for comparison. From the perspective of society as a whole, however, allocative efficiency may be more important. This is especially true when it concerns the sale of a publicly held asset to the private sector. So the seller, in this case a government, may want to choose an auction that ensures that the asset is allocated efficiently (that is, the allocation maximizes social welfare ), even if the revenue from some other, inefficient, allocation would be higher.

In this thesis we exclusively consider the single item, private values setting and concentrate our attention on maximization of social welfare as the primal goal that 
the designer wants to achieve. The issue of social welfare maximization has been studied extensively. Much research has been done on how to set the rules of the auction so that this social goal is achieved despite the fact that buyers act based on self-interest. Due to the Revelation Principle, the focus has mainly been on direct revelation mechanisms (see e.g. Mas-Colell et al. [41]). This principle states that the designer only needs to consider direct revelation mechanisms where each agent reports in a single step his valuations on all possible combinations of goods to be sold. In the private value environment the challenge is considered to be solved since in the Vickrey-Clarke-Groves (VCG) direct mechanism truth-telling is a weakly dominant strategy and the equilibrium results in an efficient allocation (Clarke [8], Groves [26], Vickrey [59]).

By construction, implementation of an equilibrium strategy in the VCG mechanism (as in any direct mechanism) requires buyers to reveal complete and exact preference information. It has been recognized however that this is an undesirable feature of a mechanism for several reasons. First, buyers may prefer not to reveal information on their valuations for reasons of privacy or long-term competitiveness (Engelbrecht-Wiggans and Kahn [17], Rothkopf et al. [52]). Second, determining one's valuation with a precision up to the last digit can be computationally demanding (Sandholm [54]). Finally, the full revelation of buyer' preferences may require a prohibitive amount of communication (Conitzer and Sandholm [13], Nisan and Segal $[45])$.

Recognition that full expression of preferences is undesirable has led to an interest in auctions where bidders need not reveal their information entirely but only partially. The challenge (also in this thesis) is to design auctions that are able to find an (approximate) efficient allocation , preferably in weakly-dominant strategies, without asking bidders to reveal complete information on their preferences.

The revelation problem is especially pronounced in settings with complex preferences involving complementarity and/or substitutability. Therefore, most results in the literature are devoted to the discussion of possible revelation reduction in combinatorial auctions. Several approaches have been proposed for limiting revelation in combinatorial auctions. Ascending combinatorial auctions (Parkes [47, 49], Ausubel and Milgrom [1], Wurman and Wellman [60]) provide one means of reducing the information requirements on bidders. Conen and Sandholm [11, 12] observe a significant topological structure in the combinatorial setting and use that to avoid asking the bidders unnecessary questions about their valuations.

Recently the preference elicitation problem has become an issue also for the setting of a single indivisible object. One general approach which allows bidders 
to retain much of their private information is to limit the number of possible bids where bidders can choose from. Blumrosen and Nisan [4] introduce one-shot auctions where bidders have to choose and announce one of $k$ price levels fixed by the auctioneer. While such simple one-shot auctions indeed achieve the objective of limiting both revelation and associated communication (compared to the direct revelation mechanism), they are not very flexible in the following sense. All players reveal their valuations with the same precision, and when doing so many players may reveal much more about their valuations than is required to determine the desired outcome. It would be better to rule out players as potential winners on the basis of less precise information on their valuation. That way one could engage only a restricted number of players in (more) precise elicitation, reducing overall revelation and incurred computation and communication cost. This leads to a natural motivation for iterative mechanisms where the players reveal their preferences incrementally, only on a need-to-know basis.

Thus, for the situation described above where $k$ prices are fixed, instead of asking players to announce one of them, the auctioneer may ask players to indicate whether they are willing to pay the price he announces. Using an increasing sequence of announced prices we eventually end up with an English auction where queries are restricted to discrete levels. This approach has received attention in auction literature though most of the works is restricted to the study of other than revelation properties of this auction. Kress and Boutilier [33] are the first who addressed revelation properties of ascending price auctions with discrete bid levels.

Reduction of preference revelation in single-item auctions is the primary focus of this thesis. It extends the idea of incremental querying and shows that by choosing carefully the query strategy, revelation of information can be significantly reduced. We propose a new iterative auction, the bisection auction, that can be used for the sale of a single indivisible object. The main idea of the proposed auction is to search for the winner and the price using the bisection method. The auction consists of several rounds in each of which the auctioneer announces the ask price and players report their demand. Depending on the players' answers in a round, the ask price of the next round can increase or decrease and some players can be eliminated from the auction. So, during each round the auctioneer asks players for very limited information about their valuation, and each successive round refines the information revealed earlier. By using a query strategy in which previously revealed information guides the selection of subsequent ask prices, elicitation in the bisection auction is focused on pertinent information. As soon as it becomes clear to the auctioneer that a particular player does not possess relevant information, i.e information which helps the auctioneer to find the winner and the price, this player 
is eliminated from the auction. Such limited participation is one of the main factors that allows us to achieve reduction of preference revelation in the bisection auction.

When evaluating the effectiveness of elicitation one generally cares about the running time, expressed in the number of queries, and/or the amount of communication required to determine an optimal (according to the specific objective of the designer) allocation. Since information about agents' valuations becomes more refined with each query, a higher number of queries leads to a better allocation. This has prompted researchers to examine the trade-off between the running time/communication complexity of query auctions and the level of allocative efficiency. In particular, several issues concerning welfare of single-item limited revelation auctions have been considered. Blumrosen et al. [4, 5] study the effect on welfare of a severe restriction of the amount of communication allowed in an auction. In the context of English auctions with discrete bid levels Rothkopf and Hastard [51] and David et al. [16] derive lower bounds on the expected loss of welfare. Parkes [50] studies a model in which bidders are uncertain about their valuations and have an option to refine their valuation for a cost. He shows that in such an environment ascending price query auctions can achieve better allocative efficiency than sealed-bid auctions, using less preference elicitation. Compte and Jehiel [9] compare the performance of iterative and sealed-bid auctions in the presence of costly information acquisition. They also support the allocative efficiency benefits of iterative auctions and show that iterative ascending auctions can avoid high information revelation but still generate a higher expected welfare than sealed bid auctions.

In this thesis, we pursue the same line of research and focus on allocative efficiency of iterative, limited revelation auctions. We analyze to what extend we can limit information revelation while still preserving full efficiency of an allocation. We show that for the case of integer valuations the proposed bisection auction, while being fully efficient, requires elicitation of much less information than the known efficient auctions. We show that in expectation only a small fraction of players' valuation information needs to be revealed before the efficient allocation can be determined. In fact for the integer setting we design an iterative implementation of the single-item VCG mechanism that finds the outcome with minimal information revelation from buyers. For the case of continuous valuations, we prove that limited information revelation is incompatible with the efficiency requirement. We show what level of revelation can be achieved if we are satisfied with a particular level of efficiency.

Part I of the thesis is devoted to the setting of integer valuations where we introduce and analyze the bisection auction. In Chapter 2 we present a full game- 
theoretic analysis of the bisection auction and show that the proposed auction is strategically equivalent to the Vickrey auction and the English auction, implying that also in the bisection auction telling the truth is a weakly dominant strategy and the equilibrium results in an efficient allocation.

While being strategically equivalent to the Vickrey and English auctions the bisection auction outperforms them in terms of information revelation and associated communication. The superiority of the bisection auction is shown in Chapter 3 where we give precise upper bounds on the expected communication and the expected information revelation of the bisection auction and compare these to lower bounds for the Vickrey and English auctions. In Chapter 4 we investigate the following questions that naturally arise: how much communication does the bisection auction need compared to any other auction that finds the Vickrey outcome, in particular, does there exist an auction with Vickrey outcome that needs less communication than the bisection auction? We introduce a measure for comparison that resembles stochastic dominance, but that, to the best of our knowledge, has not been used before for comparison of communication performance of algorithms. With respect to this measure we partially answer the posted questions. We prove for the case of two bidders a tight lower bound on the required communication and show that the bisection auction matches this bound.

Given the reported advantages of the proposed auction it seems quite attractive to put the bisection mechanism to practice. However there are some justified doubts about players' behavior in the bisection auction. Early on, it was recognized that humans have bounded rationality, e.g. due to cognitive limitations, so they do not act rationally as economic theory assumes (see Rubinstein [53], Simon [56]). The bisection auction, due to its more complicated rules, seems to be more difficult to understand and therefore it might be difficult to choose the right strategy to play. In order to investigate the practical usefulness of the bisection auction we conduct a laboratory experiment where we test and compare bidding behavior in the bisection, Vickrey and English auctions. We check whether bidders follow the dominant truth-telling strategy and how their behavior changes over time as they gain more experience. Moreover, we provide some insights concerning the efficiency of allocation and the revenue to the auctioneer. Data show that the bisection auction performs better than the Vickrey auction and only in terms of some measurements worse than the English auction. Chapter 5 describes the experiment and reports the results.

In Part II we switch to the setting of continuous valuations. In many applications there is nothing wrong with assuming discrete valuations as it is done in Part 
I. It is however sometimes desirable to be able to run an auction without an a-priori agreement on the discretization of bids. The leading example from which we draw our motivation is a computerized bidding environment in multi-agent systems. In such an environment the precision with which bidding agents represent their valuations might be unknown, and therefore preferably be left unspecified. Also time constraints can thus be captured, since time restrictions might force an auctioneer to determine valuations only up to a level of precision that is not of the same order of magnitude in which bidders do, or would like to, express their valuations. We model the possibility of arbitrary fine representation of valuations by allowing valuations to take on continuous values.

In the context of continuous valuations we investigate the allocative efficiency of query auctions. In a query auction the auctioneer sequentially queries the bidders about specific aspects of their valuations, offering them the opportunity to take one of a finite set of actions as an answer to the query. In Chapter 6 we study the limitations of query auctions regarding the objective of maximal economic efficiency. We prove that any ex-post equilibrium in an individually rational query auction that ends with positive probability after a finite number of queries cannot be fully efficient. It means that in the continuous setting query auctions, being a good tool for reducing preference revelation, do not allow achieving full allocative efficiency.

The inefficiency result implies that in the setting of continuous valuations full efficiency can only be achieved at the expense of an infinite running time of a query auction for almost all realizations of valuations. So the question arises: what price (in terms of running time) has to be paid for getting a desired level of approximate efficiency? In the last two chapters of the thesis we give an answer to this question. In Chapter 7 we introduce a very wide class of query auctions, general bisection auctions. The main characteristics of these auctions are that they have a fixed order in which active bidders are queried, they all use a binary search algorithm to determine the price, and they all stop as soon as the winner is found. We prove that a general bisection auction is indeed ex post individually rational, and there exists an ex post equilibrium, called the bluff equilibrium, which is sometimes finite. Hence in a general bisection auction the inefficiency is inevitable. In Chapter 8 we are concerned with the trade-off between running time and allocation efficiency in the bluff equilibrium of a family of general bisection auctions called $c$-bisection auctions. We show that, by choosing the appropriate parameter $c$, we can achieve arbitrary small inefficiency in equilibrium, while the running time of the auction in equilibrium is finite for all realizations of valuations. Moreover, given the desired level of efficiency, the particular choice of auction can be made independently of the number of bidders that will participate in the auction. 


\section{Part I}

\section{Bisection auctions in the discrete setting}





\section{Chapter 2}

\section{The bisection auction}

In this chapter we present a new iterative auction, the bisection auction, which can be used for the sale of a single indivisible object. The bisection auction has fewer rounds than the classical English auction and causes less information to be revealed than in the Vickrey auction. Still, it preserves all characteristics the English auction shares with the Vickrey auction: there exists an equilibrium in weakly dominant strategies in which everyone reports truthfully, in this equilibrium the object is allocated in accordance with efficiency requirements to the buyer who has the highest valuation, and the price paid by the winner of the object equals the second-highest valuation. ${ }^{1}$

\subsection{Introduction}

A well known theoretical result for private value single item auctions is strategic equivalence of the sealed bid second-price auction and the English auction. This result was shown by Vickrey [59] in his famous 1961 contribution to auction theory. Under the sealed bid second-price auction (also called the Vickrey auction) bidders are asked to submit one single sealed bid. The bidder with the highest bid is declared to be the winner. He gets the object for a price equal to the second-highest bid that is made. In the English auction ${ }^{2}$ the auctioneer calls successively higher prices. Initially all bidders are active and, as the auctioneer raises the price, they decide when to drop out. Dropping out is irrevocable so that a bidder can no longer bid on the object. The last bidder to remain is the winner and he pays the final ask price. In case where the last two bidders dropped out at the same price, a lottery can be

\footnotetext{
${ }^{1}$ The results of this chapter were first presented in Grigorieva et al. [24].

${ }^{2}$ There are several versions of the English auction. The variant described here, also called the Japanese auction, is the one that is strategically equivalent to the Vickrey auction.
} 
used to determine who gets the object.

Strategic equivalence of the two auctions implies that, as long as bidders behave rationally, in these auctions the same bidder wins and pays the same price. Two main results were established concerning these auctions. First of all, truth-telling is a weakly dominant strategy, independent of the prior distribution of the valuations of the bidders, the number of bidders or their risk attitudes. In the Vickrey auction the truth-telling strategy is to submit a bid equal to one's private value. In the English auction the truth-telling strategy is to remain active until one's value is reached. Secondly, the resulting truth-telling equilibrium is efficient, meaning that the object gets assigned to the bidder who values it most.

Yet both the Vickrey auction and the English auction have their drawbacks in practical use. As any direct mechanism the Vickrey auction requires all participants to reveal complete and exact preference information. It has been recognized that the full revelation of bidders' preferences is not necessarily a desirable feature of a mechanism. ${ }^{3}$

First, buyers may prefer not to reveal information on their valuations for reasons of privacy or long-term competitiveness (see e.g. Engelbrecht-Wiggans and Kahn [17], Rothkopf et al. [52]). Buyers might be reluctant to truthfully reveal their full private value if there will be subsequent auctions or negotiations in which the information revealed can be used against them. For example, after such an auction the buyer will be at a disadvantage in future negotiations with the seller who may be able to extract more surplus using information about the buyer's valuation. ${ }^{4}$

Second, determining one's valuation with a precision up to the last digit can be computationally demanding, see Sandholm [54]. A buyer may have to spend effort in determining his unknown a priori preferences by computing (see e.g. Larson and Sandholm [37, 36], Parkes [48]) or by gathering additional information (see e.g. Bergmann and Välimäki [3], Compte and Jehiel [10]).

Finally, the full revelation of buyer' preferences may require a prohibitive amount of communication (see e.g. Conitzer and Sandholm [13], Nisan and Segal [45]). For example in a combinatorial auction which allocates heterogeneous indivisible items

\footnotetext{
${ }^{3}$ For extensive discussion of other weaknesses and disadvantages of the VCG mechanism see e.g. Ausubel and Milgrom [1, 2].

${ }^{4}$ One can argue that recently a great deal of research in computer science has been done in developing cryptographic protocols for auctions that preserve the privacy of the participants. Various schemes to ensure the safe (from a privacy perspective) conduction of sealed-bid auctions have been proposed, see Brandt [6], Naor et al. [44]. Indeed, in some settings by the use of the proposed protocols we can ensure the confidentiality of the bids, but the problems of computing and communicating the bids remain to be solved.
} 
among bidders whose preferences for bundles of items can exhibit complementarities, every buyer has to report his valuation for each subset of the items, and the number of such subsets is exponential in the number of items. With 30 items, full revelation of such preferences would require the communication of more than one billion numbers.

A significant problem with the English auction is that it might proceed at a very slow pace: if a very small price increment is chosen, in which case the auction might take a long and unpredictable amount of time to reach the required price level. This shortcoming can be devastating, for instance, when using auctions to allocate timeshared resources in real-time environments. One remedy is to introduce a fixed bid increment, as has been done for example in the spectrum auctions (see e.g. Moldovanu and Jehiel [43], van Damme [58]). This though is known to affect the efficiency of the auction (Rothkopf and Harstad [51]).

Attempts to speed up the auction while preserving efficiency were made in Fujishima et al.[19]. The authors introduce a class of multi-round sealed-bid auctions, called survival auctions, and show that a special case, the $(n-1)$ round survival auction is strategically equivalent to the English auction and therefore preserves its equilibrium properties. In the proposed auction bidders submit a sealed-bid in each round. All except a bidder who submits the lowest bid survive the round. The losing bid is announced to be the minimum bid for the next round. The last bidder to remain is the winner and he pays the final losing bid. The auction of $n$ bidders predictably takes $n-1$ rounds which makes a significant improvement in speed in comparison to the English auction. Having quick and predictable termination time, the survival auction, however, requires the full and complete revelation of bidders' valuation, the same as the Vickrey auction.

So, the question that arises is: how to design an auction that elicits less information about bidders' valuations than the Vickrey auction but still enough to guarantee an efficient allocation and which is at the same time faster than the English auction. Based on Milgrom and Weber [42], limited information revelation is often believed to be incompatible with the efficiency requirement. Nevertheless, the primary contribution of this chapter is to present and to analyze an alternative auction format, called the bisection auction, that possesses these properties for the case of selling a single indivisible object under private values. 


\subsection{The rules of the bisection auction}

Suppose a single indivisible object is auctioned. The buyers' valuations are assumed to be integer, randomly drawn from a bounded interval - by default of the form $\left[0,2^{R}\right)$ for some positive integer $R$. The bisection auction has $R$ rounds. The price sequence starts at the middle of the initial interval with a price equal to $2^{R-1}$. Bidders report their demand at the current price by sealed bids. A yes-bid stands for the announcement to be willing to buy at the current price, a no-bid for the contrary. As a function of these bids, the auctioneer announces the price of the next round.

In case there are at least two players submitting a yes-bid, the price goes up to the middle of the upper half interval, i.e., the interval $\left[2^{R-1}, 2^{R}\right)$. The players that are allowed to participate actively in the next round are the ones that said yes and they are competing for the object in the price range $\left[2^{R-1}, 2^{R}\right)$. The other players drop out of the auction and do no longer have any influence on the proceedings of the auction. In case there is at most one player saying yes, attention shifts to the lower half interval, i.e. the interval $\left[0,2^{R-1}\right)$ and the price goes down to the middle of this interval. Two different things can happen now. First, the easy case, if no-one has submitted a yes-bid. In that case all active players remain active in the next round. In the other case there is a single player that submitted a yes-bid. This player now becomes the winner and he gets the object. Nevertheless the auction does not end, but enters a price-determination phase. The active players in the next round are the ones that were active in the previous round minus the winner. In order to keep active players motivated to participate in the auction they should not get to know that the object has already been assigned. Therefore we assume that bidders aren't able to observe bids of the others. The remaining active players are competing on the lower half interval $\left[0,2^{R-1}\right)$. The winner, although he is no longer considered to be active, is considered to say yes to all prices that are proposed beyond the moment he became the winner. After all, all these prices will be lower than the price he agreed to when he became the winner. Apart from this, the way it is decided whether the price should go up or down is not any different from the way this is decided in the winner-determination phase. In each round depending on submitted bids we subsequently restrict attention to either the lower half of the current interval, or to the upper half of the current interval.

Iterating this procedure will eventually yield a winner and a price. If in no round there was precisely one player that said yes then ties are broken by random assignment to a player who is still active after $R$ rounds. At no point during the auction are the bidders informed that the object has been assigned to the winning 
bidder, or indeed that any particular bidder has dropped out of the auction. The price is uniquely determined because in each round the length of the current interval goes down by one half. Since the initial interval is of length $2^{R}$, after $R$ rounds the resulting interval is of length 1 . And since it is a half-open interval, it contains exactly one integer. This integer is declared to be the price the winner of the auction has to pay for the object.

The following example illustrates how the bisection auction works.

Example. Suppose there are four bidders, A, B, C, and D, with the following integer private valuations from the interval $[0,16): 11,4,15,9$. To determine the winner and the price in this setting the bisection auction takes four rounds and starts with an ask price equal to 8. Suppose that each bidder chooses to respond truthfully and follows a straightforward strategy under which he says yes if an ask price is less or equal to his valuation and no otherwise. Bidders are not informed about other bidders' choices. The bisection auction proceeds as follows:

\begin{tabular}{cccccccc}
\hline Round & Price & $\begin{array}{c}\text { Lower } \\
\text { bound }\end{array}$ & $\begin{array}{c}\text { Upper } \\
\text { bound }\end{array}$ & $\begin{array}{c}\text { Bidder A } \\
v_{A}=11\end{array}$ & $\begin{array}{c}\text { Bidder B } \\
v_{B}=4\end{array}$ & $\begin{array}{c}\text { Bidder C } \\
v_{C}=15\end{array}$ & $\begin{array}{c}\text { Bidder D } \\
v_{D}=9\end{array}$ \\
\hline 1 & 8 & 0 & 16 & yes & no & yes & yes \\
2 & 12 & 8 & 16 & no & $($ no $)$ & yes & no \\
3 & 10 & 8 & 12 & yes & $($ no) & $($ yes $)$ & no \\
4 & 11 & 10 & 12 & yes & (no) & $($ yes $)$ & $($ no $)$ \\
\hline
\end{tabular}

Since three bidders submitted yes-bids in the first round, the price increases to the middle of the current price and the current upper bound. So the ask price of the second round is 12 . These three bidders remain active while bidder B drops out. We allow a drop-out to submit any bid, but consider any bid of a drop-out as a no-bid. Since there is only one yes-bid in the second round we have a winner and we enter what we call the price determination phase. From now on, any bid of the winner, bidder $\mathrm{C}$, is considered as a yes-bid. Players A and D are still active. In the third round, there are two yes-bids so the price increases. Player D drops out. In the fourth round, the auction terminates. Taking into account bids made during the last round we compute the final lower and upper bounds. Since there were 2 yes-bids the upper bound remains 12 while the lower bound becomes 11 . The winner, bidder $\mathrm{C}$, takes the object and pays price 11 which is the smallest Walrasian price for the demand announced by the bidders that participated in this auction.

A related auction format is mentioned in Fujishima et al. [19] in the context of designing iterative auctions with quick and predictable termination time. In particular they write: "In what we call "the binary price-search auction", the auctioneer 
queries all bidders whether they are willing to pay a given price. If only one bidder answers yes, he gets the good at that price. If zero or more than one answer yes, another query follows at a lower or higher price, in such a way to converge at logarithmic speed to a price that exactly one bidder will be willing to pay. Unfortunately, this and other "accelerated auctions" are difficult to analyze with the tools of game theory." The binary price-search auction is very similar to the bisection auction. A subtle difference is that the binary price-search auction stops as soon as the winner has been found while the bisection auction continues with a pricedetermination phase. Exactly the presence of this second phase makes the auction incentive compatible as we show in the this chapter.

\subsection{Formal representation of the auction}

In section 2.2 we already gave a description of the bisection auction. A formal proof of strategic equivalence requires an exact framework. For this reason we will represent the bisection auction by an extensive form game. Formally we would need to introduce a move of nature that represents the realization of players' valuation, see e.g. Osborne and Rubinstein [46]. But to simplify the analysis we deviate somewhat from the classical approach to games of this sort. We will not include a move of nature in the tree to represent the realization of the valuations but rather construct the tree independent of these realizations. Next, notice that the rules of the auction require players to move simultaneously in each round. However, in an extensive form setting this is not explicitly possible but takes the form of sequential moves in which players do not observe moves made by the other players. Thus we model the auction as a non-cooperative game in extensive form with imperfect information.

\section{The game}

(1) A finite set $N=\{1, \ldots, n\}$ of players that participate in the game.

(2) A number of rounds $R$ that specifies the duration of the game.

(3) For each player a decision set $A=\{y e s, n o\}$. This reflects the fact that each player has to make a binary decision in each and every of his information sets.

(4) Every node in the game tree is a vector $a$ that represents the history of decisions chosen by players before the game reached this node. Formally, $a=\left(a_{k}\right)_{k=1}^{r}$ with $1 \leq r \leq R$ where $a_{k}=\left(a_{k i}\right)_{i=1}^{n}$ for $k<r$ and $a_{r}=\left(a_{r i}\right)_{i=1}^{j}$ for some $j \leq n$. Here $a_{k i}$ is the decision in $A$ by player $i$ in round $k$.

The length of a node is defined as $l(a)=(r-1) n+j$. The initial node $a_{0}$ is a 
node with length equal to 0 . The nodes with length equal to $r n$ are referred to as the start of round $r+1$. The terminal nodes are nodes whose length is equal to $R n$.

(5) There is an edge between two nodes $a$ and $b$ if the length of $b$ is equal to the length of $a$ plus one, and for all $i$ and $k$ for which $a_{k i}$ is defined, $a_{k i}=b_{k i}$.

Let $X_{r}$ denote the set of nodes corresponding to round $r$. The set $X_{r}$ partitions into $n$ sets $X_{r i}$, each $X_{r i}$ being the set of nodes in round $r$ where player $i$ has to make a decision. Formally, $X_{r i}=\{a \mid l(a)=(r-1) n+i-1\}$. For a node $a \in X_{r}$ and $q<r$, let $a^{q}=\left(a_{k}\right)_{k=1}^{q}$ denote the part of the history $a$ corresponding to the first $q$ rounds. We associate with every node $a \in X_{r}$ the set $P(a)$ of players whose decisions made in round $r-1$ equal yes, taking into account that decisions of drop-outs are counted as no, decisions of a winner as yes.

(6) Node $a$ in $X_{r i}$ belongs to the information set $H(a)$ defined as the set of all nodes $b$ from $X_{r i}$ such that for all $k<r$

1. $a_{k i}=b_{k i}$

2. $\left|P\left(a^{k}\right)\right| \leq 1$ iff $\left|P\left(b^{k}\right)\right| \leq 1$.

(7) We associate with each terminal node $\tau$, a set $W I N(\tau)$ that is a set of candidate winner(s) of the game if the game terminates in node $\tau$. If in one of the rounds corresponding to node $\tau$ there has been exactly one yes-bid, then $W I N(\tau)$ consists of the player that submitted this bid. Otherwise, $W I N(\tau)$ equals the set of players that never dropped out. In that case, the winner of the game is determined by a lottery among all players $i$ in $W I N(\tau)$. The price the winner pays is

$$
p(\tau)=2^{R-1}+\sum_{k=1}^{R-1} \lambda_{k} 2^{R-k-1}+\beta
$$

where for all $k$

$$
\lambda_{k}=\left\{\begin{aligned}
-1 & \text { if }\left|P\left(\tau^{k}\right)\right| \leq 1 \\
1 & \text { otherwise }
\end{aligned} \quad \text { and } \quad \beta=\left\{\begin{aligned}
-1 & \text { if }|P(\tau)| \leq 1 \\
0 & \text { otherwise }
\end{aligned}\right.\right.
$$

The payoff of player $i$ with private value $v_{i}$ in terminal node $\tau$ is defined by

$$
U_{i}(\tau)= \begin{cases}v_{i}-p(\tau) & \text { with probability } \frac{1}{|W I N(\tau)|} \text { if } i \in W I N(\tau) \\ 0 & \text { else. }\end{cases}
$$

A few remarks. To describe the auction more precisely, we partition the set of players $N$ into three subsets of players, $A(a)$ - the set of players that are active in node $a, W(a)$ - the winner set in node $a$, and $D(a)$ - the set of players that dropped 
out before the game reached node $a$. For the initial node $a_{0}$ they are defined as follows: $A\left(a_{0}\right)=N, W\left(a_{0}\right)=\emptyset$ and $D\left(a_{0}\right)=\emptyset$. We iteratively define them for nodes with arbitrary length. For a node $a=\left(a_{k}\right)_{k=1}^{r}$ with $l(a)=n r$,

$$
\begin{aligned}
& W(a)=\left\{\begin{array}{lll}
W\left(a^{r-1}\right) & \text { if } \quad W\left(a^{r-1}\right) \neq \emptyset \\
\emptyset & \text { if } \quad W\left(a^{r-1}\right)=\emptyset \text { and }|P(a)| \neq 1 \\
P(a) & \text { if } \quad W\left(a^{r-1}\right)=\emptyset \text { and }|P(a)|=1
\end{array}\right. \\
& D(a)= \begin{cases}D\left(a^{r-1}\right) \\
D\left(a^{r-1}\right) \cup\left\{i \in A\left(a^{r-1}\right) \mid a_{r i}=n o\right\} & \text { otherwise }\end{cases} \\
& A(a)=N \backslash(W(a) \cup D(a)) .
\end{aligned}
$$

For a node $a=\left(a_{k}\right)_{k=1}^{r}$ with $l(a) \neq n r$, we define these sets as follows: $W(a)=$ $W\left(a^{r-1}\right), D(a)=D\left(a^{r-1}\right)$, and $A(a)=A\left(a^{r-1}\right)$.

In a similar way as for terminal nodes, we can associate a price with an arbitrary internal node. For a node $a=\left(a_{k}\right)_{k=1}^{r}$ with $l(a)=n r$ we define its price by

$$
p(a)=2^{R-1}+\sum_{k=1}^{r} \lambda_{k} 2^{R-k-1}
$$

where for all $k$

$$
\lambda_{k}=\left\{\begin{aligned}
-1 & \text { if }\left|P\left(a^{k}\right)\right| \leq 1 \\
1 & \text { otherwise }
\end{aligned}\right.
$$

Note that for $a$ with $l(a)=n r$ the price $p(a)=p\left(a^{r-1}\right)+2^{R-r-1}$ if $|P(a)|>1$ and $p(a)=p\left(a^{r-1}\right)-2^{R-r-1}$ if $|P(a)| \leq 1$. Furthermore, for a node $a=\left(a_{k}\right)_{k=1}^{r}$ with $l(a) \neq n r$ we define $p(a)=p\left(a^{r-1}\right)$.

In any node $a$ we now have a price. The actions a player can take in this node $a$, either a yes or a no, can be interpreted as being his answer to the question:

Are you willing to pay the price $p(a)$ for the object we are selling?

Moreover, these prices can be used to characterize information sets. For any $a, b \in X_{r i}$ from the same information set, $p(a)=p(b)$. Each information set in $X_{r i}$ can be represented by its associated price and the sequence of decisions chosen by player $i$ in the first $r-1$ rounds. 
Observe that the way the auction proceeds depends only on the behavior of active players. The following result shows that the bisection auction leads to a very particular information structure.

Proposition 2.3.1. For an information set in which player $i$ is active and for every decision of player $i$ made in this set, there exists exactly one immediate successor information set $H(a)$ in which player $i$ is still active. Moreover, this immediate successor is, in case the player chose yes, the one with $|P(a)|>1$ and the one with $|P(a)| \leq 1$ in the other case.

This proposition is an immediate consequence of the definition of an information set. Once a player said no from the fact that he stays active he can infer that there was nobody or exactly one player with a yes-bid, but he can't distinguish between these two possible cases so that he can conclude whether the winner is found already.

\subsection{Playing the game}

\subsubsection{Equivalence of strategies and threshold strategies}

Let $\mathcal{H}_{i}$ denote the collection of player $i$ 's information sets.

Definition 2.4.1. A strategy for player $i$ is a function $s_{i}: \mathcal{H}_{i} \rightarrow A$.

First notice that the number of information sets of each player corresponding to round $k$ is equal to $4^{k-1}$ (unless the number of players equals two, in which case it is $\left.3^{k-1}\right)$. The total number of information sets in the game for each player is equal to $\sum_{k=1}^{R} 4^{k-1}=\frac{1}{3}\left(4^{R}-1\right)$. Thus, the number of possible strategies of a player equals $2^{\frac{1}{3}\left(4^{R}-1\right)}$. We will show that the number of essentially different strategies is much lower, in the order of $2^{R}$ to be specific. We denote by $p(H)$ the price corresponding to information set $H$.

Definition 2.4.2. Let $t$ be an integer, called the threshold. The threshold strategy $s_{i}^{t}: \mathcal{H}_{i} \rightarrow A$ assigns the action yes to $H \in \mathcal{H}_{i}$ with $p(H) \leq t$ and the action no to $H \in \mathcal{H}_{i}$ with $p(H)>t$.

We show next that from a strategic perspective players may restrict themselves to using threshold strategies only. First we need the following result.

Lemma 2.4.3. Let $s_{i}$ be a strategy of player $i$ and let $r$ be a round. Then there is a unique information set $H_{r, i}\left(s_{i}\right)$ of player $i$ such that if he is still active in round 
$r$ he will necessarily be in information set $H_{r, i}\left(s_{i}\right)$. Furthermore, the price of this information set $H_{r, i}\left(s_{i}\right)$ equals

$$
p\left(H_{r, i}\left(s_{i}\right)\right)=2^{R-1}+\sum_{k=1}^{r-1} \lambda_{k} 2^{R-k-1}
$$

where for all $k$

$$
\lambda_{k}=\left\{\begin{array}{rll}
-1 & \text { if } \quad s_{i}\left(H_{k, i}\left(s_{i}\right)\right)=\text { no } \\
1 & \text { if } \quad s_{i}\left(H_{k, i}\left(s_{i}\right)\right)=\text { yes }
\end{array}\right.
$$

Proof. For round $r=1$. The information set $H_{1, i}\left(s_{i}\right)$ is simply the unique information set $X_{1, i}$ of player $i$ in round 1 . Its price is $p\left(H_{1, i}\left(s_{i}\right)\right)=2^{R-1}$.

Now suppose that for some $r<R$ the information set $H_{r, i}\left(s_{i}\right)$ has been reached (and its price is determined as above). Since player $i$ is supposed to be active in this information set, according to Proposition 2.3.1 the action $s_{i}\left(H_{r, i}\left(s_{i}\right)\right)$ player $i$ takes in this information set determines a unique immediate successor $H_{r+1, i}\left(s_{i}\right)$ in which player $i$ is still active.

Furthermore, for $s_{i}\left(H_{r, i}\left(s_{i}\right)\right)=n o$, this immediate successor is one with $|P(a)| \leq$ 1. This implies that the price of $H_{r+1, i}\left(s_{i}\right)$ is equal to $p\left(H_{r, i}\left(s_{i}\right)\right)-2^{R-r-1}$. Thus, $\lambda_{r}=-1$ in this case. If on the other hand $s\left(H_{r, i}\left(s_{i}\right)\right)=y e s$, this immediate successor is one with $|P(a)|>1$. This implies that the price of $H_{r+1, i}\left(s_{i}\right)$ is equal to $p\left(H_{r, i}\left(s_{i}\right)\right)+2^{R-r-1}$ and hence $\lambda_{r}=1$.

This result enables us to specify for any strategy its equivalent threshold strategy.

Definition 2.4.4. Let $s_{i}$ be a strategy of player $i$. The threshold $t_{i}\left(s_{i}\right)$ is defined by $t_{i}\left(s_{i}\right)=p\left(H_{R, i}\left(s_{i}\right)\right)+\beta$ where $\beta=-1$ if $s_{i}\left(H_{R, i}\left(s_{i}\right)\right)=n o$ and $\beta=0$ if $s_{i}\left(H_{R, i}\left(s_{i}\right)\right)=$ yes.

The following result implies that a strategy $s_{i}$ and the threshold strategy $s_{i}^{t_{i}\left(s_{i}\right)}$ are realization-equivalent.

Theorem 2.4.5. Let $s_{i}$ be a strategy and let $\widetilde{s}_{i}$ be the threshold strategy with threshold $t_{i}\left(s_{i}\right)$. Let $s_{-i}$ be an arbitrary collection of strategies for players other than $i$. Let $a$ be the realized history if $s=\left(s_{i}, s_{-i}\right)$ is played and let $b$ be the realized history if $\widetilde{s}=\left(\widetilde{s_{i}}, s_{-i}\right)$ is played. Then for both histories holds

1. For every round $r, A\left(a^{r-1}\right)=A\left(b^{r-1}\right), D\left(a^{r-1}\right)=D\left(b^{r-1}\right), W\left(a^{r-1}\right)=W\left(b^{r-1}\right)$.

2. For every round $r$ where player $i$ is active according to a we have $a^{r}=b^{r}$.

3. For all $k \leq r$ and all players $j \neq i, a_{k j}=b_{k j}$.

4. All players $j \neq i$ in all rounds reach the same information sets.

5. The payoff of all players are the same in a and $b$. 
Proof. We will first prove statements 1, 2 and 3 by induction.

(I) For $r=1$. By definition, $A\left(a^{0}\right)=A\left(b^{0}\right), D\left(a^{0}\right)=D\left(b^{0}\right)$ and $W\left(a^{0}\right)=W\left(b^{0}\right)$.

In order to prove (2) and (3), notice that the set $X_{1 j}$ is the only information set of any player $j$ in round 1 . Obviously $a_{1 j}=b_{1 j}$ for all $j \neq i$, so it remains to prove that $a_{1 i}=b_{1 i}$ or equivalently that $s_{i}\left(X_{1 i}\right)=\tilde{s}_{i}\left(X_{1 i}\right)$.

If $s_{i}\left(X_{1 i}\right)=$ no we have to show that $t_{i}\left(s_{i}\right)<p\left(X_{1 i}\right)$. By Lemma 2.4.3 it holds that $\lambda_{1}=-1$, so

$$
t_{i}\left(s_{i}\right)=2^{R-1}+\sum_{k=1}^{R-1} \lambda_{k} 2^{R-k-1}+\beta \leq p\left(X_{1 i}\right)-2^{R-2}+\sum_{k=2}^{R-1} 2^{R-k-1}<p\left(X_{1 i}\right) .
$$

Similarly, if $s_{i}\left(X_{1 i}\right)=$ yes we can show that $t_{i}\left(s_{i}\right) \geq p\left(X_{1 i}\right)$.

(II) Now suppose that (1), (2) and (3) are true in round $r$. We will show that they are also true for $r+1$. Using (3) of the induction hypothesis it follows easily that $A\left(a^{r}\right)=A\left(b^{r}\right), D\left(a^{r}\right)=D\left(b^{r}\right)$ and $W\left(a^{r}\right)=W\left(b^{r}\right)$, which proves (1).

In order to prove (2) and (3), suppose that according to $a$ player $i$ is active in round $r+1$. Then player $i$ is also active in round $r$. We know from the induction hypothesis that $a^{r}=b^{r}$. It is then clear that $a_{r+1, j}=b_{r+1, j}$ for all players $j \neq$ $i$. The only thing left to show is $a_{r+1, i}=b_{r+1, i}$ or equivalently $s_{i}\left(H_{r+1, i}\left(s_{i}\right)\right)=$ $\widetilde{s}_{i}\left(H_{r+1, i}\left(s_{i}\right)\right)$.

If $s_{i}\left(H_{r+1, i}\left(s_{i}\right)\right)=n o$, we have to show that $t_{i}\left(s_{i}\right)<p\left(H_{r+1, i}\left(s_{i}\right)\right)$. Lemma 2.4.3 implies $\lambda_{r+1}=-1$. So,

$$
\begin{aligned}
t_{i}\left(s_{i}\right) & =p\left(H_{r+1, i}\left(s_{i}\right)\right)+\sum_{k=r+1}^{R-1} \lambda_{k} 2^{R-k-1}+\beta \\
& \leq p\left(H_{r+1, i}\left(s_{i}\right)\right)-2^{R-r-2}+\sum_{k=r+2}^{R-1} 2^{R-k-1}<p\left(H_{r+1, i}\left(s_{i}\right)\right) .
\end{aligned}
$$

The other case goes again along the same lines of reasoning.

(4) This follows easily from (2).

(5) Observe that the payoff is a function of the information sets reached in round $R$ and decisions of active players made in the last round. All these are the same. 
Corollary 2.4.6. Any strategy $s_{i}$ of a player $i$ can be represented by an equivalent threshold strategy $\widetilde{s_{i}}$.

In the following, we will restrict our attention to threshold strategies and denote them just by referring to the threshold.

\subsubsection{Playing the game with threshold strategies}

In this subsection we show that the winner of the object to be sold is a player with the maximum threshold and the price equals the second-highest threshold, and consequently that truth-telling is a weakly dominant strategy.

Let $t=\left(t_{i}\right)_{i \in N}$ be a profile of thresholds played in the bisection auction. This profile remains fixed during the next few statements. We denote the terminal node where the game ends according to this profile by $\tau^{*}$. Let $p(r)=p\left(\left(\tau^{*}\right)^{r-1}\right)$ be the price in round $r$ for this realization of the game.

Definition 2.4.7. The maximum threshold $t_{\max }$ is defined to be the number $\max _{i \in N} t_{i}$.

Definition 2.4.8. Let $k$ be a player with $t_{k}=t_{\max }$. The second-highest threshold $t_{\text {sec }}$ is defined to be the number $\max _{i \in N /\{k\}} t_{i}$.

Theorem 2.4.9. Let $t=\left(t_{i}\right)_{i \in N}$ be a profile of thresholds played in the bisection auction. The winner of the game is necessarily a player whose threshold equals the maximum threshold.

Proof. According to the definition the set of candidate winner(s) of the game is $W I N\left(\tau^{*}\right)=W\left(\tau^{*}\right)$ if $W\left(\tau^{*}\right) \neq \emptyset$ and $W I N\left(\tau^{*}\right)=A\left(\tau^{*}\right)$ otherwise.

Case 1. $W\left(\tau^{*}\right) \neq \emptyset$. Let $W\left(\tau^{*}\right)=\{k\}$. Consider the round $r$ in which player $k$ became the winner. Then $a_{r k}=y e s$ and $a_{r j}=n o$ for all $j \neq k$. Thus $t_{j}<p(r)$ for all $j \neq k$ and $t_{k} \geq p(r)$.

Case 2. $W\left(\tau^{*}\right)=\emptyset$. Note that all players in $A\left(\tau^{*}\right)$ must have chosen the same action in each round. Therefore they have the same thresholds. A player $j$ who became inactive in some round $r$ must have said no in this round while all players in $A\left(\tau^{*}\right)$ said yes. But then $t_{j}<p(r) \leq t_{k}$ for all $k \in A\left(\tau^{*}\right)$ and for all $j \notin A\left(\tau^{*}\right)$.

Lemma 2.4.10. Let $t=\left(t_{i}\right)_{i \in N}$ be a profile of thresholds played in the bisection auction. A player with the second highest threshold remains active till the end of the game. 
Proof. Let player $j$ be a player with the second highest threshold. We have to show that $j \in A\left(\tau^{r}\right)$ for all $0 \leq r \leq R$. The case $r=0$ is trivial. Suppose the statement is true for some $r$. We show that it is true for $r+1$. There are three situations concerning the winner set that could possibly occur.

(A) $W\left(\tau^{r+1}\right)=\emptyset$. It means that $\left|P\left(\tau^{r+1}\right)\right| \neq 1$. Suppose $\left|P\left(\tau^{r+1}\right)\right|=0$. Then $A\left(\tau^{r+1}\right)=A\left(\tau^{r}\right)$ - all active players remained active, thus player $j$ too. Suppose $\left|P\left(\tau^{r+1}\right)\right|>1$. Then every player with the highest threshold, as well as any player with the second highest threshold, decides yes. In this case all players from $A\left(\tau^{r}\right)$ with a yes-bid remain active, including player $j$.

(B) $\quad W\left(\tau^{r}\right)=\emptyset$ and $W\left(\tau^{r+1}\right) \neq \emptyset$. It means that $\left|P\left(\tau^{r+1}\right)\right|=1$. The only yes-bid comes from the, in this case unique, player with the highest threshold, and he becomes the winner. The set of active players is $A\left(\tau^{r+1}\right)=A\left(\tau^{r}\right) / W\left(\tau^{r}\right)$. Thus player $j$ remains active.

(C) $\quad W\left(\tau^{r}\right) \neq \emptyset$. For this case it holds that $\left|P\left(\tau^{r+1}\right)\right| \geq 1$. Suppose $\left|P\left(\tau^{r+1}\right)\right|=$ 1. Then all players from $A\left(\tau^{r}\right)$ have made a no-bid and remain active, thus player $j$ too. If $\left|P\left(\tau^{r+1}\right)\right|>1$, then at least one player from $A\left(\tau^{r}\right)$ has made a yes-bid and remains active. This surely includes player $j$ because he has the highest threshold among the players that are still active.

Lemma 2.4.11. Let $t=\left(t_{i}\right)_{i \in N}$ be a profile of thresholds played in the bisection auction. The price of the terminal node $\tau^{*}$ is equal to the threshold of any player that is active at the end of the game.

Proof. Consider a player from $A\left(\tau^{*}\right)$, say $i$. First of all we will show that $\left|t_{i}-p(R)\right| \leq$ 1. We prove this inductively by showing that $\left|t_{i}-p(r)\right| \leq 2^{R-r}$ for each round $r$. For $r=1$ the statement follows easily. Suppose it is true for some $r$.

(a) $\quad p(r) \leq t_{i}$. Together with the assumption of the induction hypothesis it gives us $p(r) \leq t_{i} \leq p(r)+2^{R-r}$. Moreover, in this case player $i$ has made a yes-bid in round $r$. Since he is active in all rounds, the price went up to $p(r)+2^{R-r-1}$ in round $r+1$, so $\left|t_{i}-p(r+1)\right| \leq 2^{R-r-1}$.

(b) $p(r)>t_{i}$. For this situation we show in the same way that $p(r)-2^{R-r} \leq t_{i} \leq$ $p(r)$ and $p(r+1)=p(r)-2^{R-r-1}$ from which it follows that $\left|t_{i}-p(r+1)\right| \leq 2^{R-r-1}$.

Thus, $\left|t_{i}-p(R)\right| \leq 1$ for any player who is active at the end of the game.

Case 1. $\quad p\left(\tau^{*}\right)=p(R)-1$ and so $\left|P\left(\tau^{*}\right)\right| \leq 1$. This last inequality implies that player $i$ said no in the last round, so $p(R)>t_{i}$. Since $\left|t_{i}-p(R)\right| \leq 1, p\left(\tau^{*}\right)=t_{i}$.

Case 2. $\quad p\left(\tau^{*}\right)=p(R)$ and so $\left|P\left(\tau^{*}\right)\right|>1$. This last inequality implies that 
player $i$ said yes in the last round, in other words, $p(R) \leq t_{i}$. If, he said yes in all rounds, $p(R)=2^{R}-1$, and thus $p(R)=t_{i}$. Otherwise, if follows from the construction of the bisection auction that the last round in which player $i$ has said no has a price equal to $p(R)+1$. So $p(R) \leq t_{i}<p(R)+1$, and thus $p\left(\tau^{*}\right)=t_{i}$.

Corollary 2.4.12. Let $t=\left(t_{i}\right)_{i \in N}$ be a profile of thresholds played in the bisection auction. The price the winner of the game pays is equal to the second highest threshold.

Definition 2.4.13. The truth-telling strategy of player $i$ is the threshold strategy for which $t$ is equal to player $i$ 's valuation $v_{i}$.

Theorem 2.4.14. The truth-telling strategy of player $i$ is a weakly dominant strategy.

Proof. From the previous results, specifically Theorem 2.4.9 and Corollary 2.4.12, it follows that we can interpret the thresholds from Corollary 2.4.6 as bids in the Vickrey auction. The bisection auction is therefore strategically equivalent to the Vickrey auction, and telling the truth is a weakly dominant strategy.

A final immediate consequence of this theorem is the following corollary.

Corollary 2.4.15. The truth-telling strategy profile constitutes a symmetric Nash equilibrium. It is the unique perfect Nash equilibrium.

\subsubsection{Some remarks on generalizations of the bisection auction}

The bisection auction assumes that the bidders' valuations are randomly drawn from a probability distribution on the bounded set $\left[0,2^{R}\right)^{n}$. There is no problem to extend the bisection auction to the case where the upper bound is equal to infinity. The key insight is that the current construction where after each round the current interval is split in two equally sized intervals can be generalized to any split without changing our main results. In particular, one may start the first round with the interval $[0, \infty)$, which splits in the intervals $[0, u)$ and $[u, \infty)$ for some integer $u$. As long as all realizations of valuations are assumed to be finite, after a finite number of rounds attention will be restricted to an interval with a finite upper bound.

The rules of the bisection auction presented in the sections above are constructed in such a way that the only information revealed to players after each round is the change of the price. This information policy is less restrictive than it may look at first sight. First of all, in order to make the formal representation of the auction and the proofs of its properties concise and comprehensive we decided not to include the number or identities of the drop-outs in the information revealed. Notice however 
that revealing which players dropped out does not reveal whether or when the winner has been determined. Thus, revealing which players drop out does not change the weak dominance of truth-telling. Only the formal proof of this property becomes more sophisticated. Information sets of a player are characterized then, in addition to price and own previous actions, by time and the identity of the players that dropped out. So, the strategy space becomes larger and more complicated. But we still can show that for any strategy from this extended space there exists an outcome equivalent threshold strategy, so that the results of this chapter also hold for the auction with such an information policy.

The possibilities offered by for instance the internet render a practical implementation of the bisection auction feasible. Bidders should be informed about the rules of the bisection auction, in particular about how prices respond to the number of yes-bids, and about the fact that the price in each round is the only information transmitted to them.

Now we will examine what happens if after each round the bids of all the players are revealed, so not only the drop-outs but also the winner becomes publicly known. The following example shows that truth-telling is not a weakly dominant strategy in the bisection auction under this information policy.

Example. Consider the bisection auction under the full information policy with two players. Let player A choose to act according to the following strategy: to say no up to the moment the winner is found and yes afterwards. Regardless of his valuation the best that player $\mathrm{B}$ can do against this strategy is to say no in all rounds but the very last one. Player $\mathrm{B}$ will get the object for a price equal to zero.

Nevertheless, it can still be shown that the truth-telling profile constitutes an ex-post Nash equilibrium. Indeed, let us analyze what the best thing is player $i$ can do given that all other players follow the truth-telling strategy. If player $i$ does not win, his payoff is zero. If he wins he pays the price equal to the highest valuation of other players, so the price is not influenced by his behaviour during the auction. Thus, any strategy that guarantees winning in case player $i$ 's valuation is the highest is a best response. One such strategy is the truth-telling strategy.

\subsection{Conclusion}

We proposed a new auction, the bisection auction, and analyzed its equilibrium properties. First, we have proved that in the bisection auction, threshold strategies are sufficient from a strategic point of view. Furthermore, we have shown strategic 
equivalence of the bisection auction to the Vickrey auction. Using this result we established that the proposed auction is incentive compatible, that is truth-telling is a weakly dominant strategy. The equilibrium that results when everyone tells the truth is efficient in the sense that the player with the highest valuation gets the object. 


\section{Chapter 3}

\section{Bounds on communication performance}

In this chapter we discuss the information revelation requirements of the bisection auction and the associated amount of data that needs to be transmitted. We show that in the truthtelling equilibrium the bisection auction is economical in its demand for information on the valuations of the players. It requires the players to transmit less information bits to the auctioneer than the Vickrey and English auctions. ${ }^{1}$

\subsection{Introduction}

It has been shown in the previous chapter that the bisection auction is strategically equivalent to the Vickrey and English auctions and therefore it preserves efficiency and has truth-telling as a dominant strategy.

While being strategically equivalent to the Vickrey and English auctions the bisection auction outperforms them in terms of valuation revelation requirement. In comparison with the Vickrey and English auctions in the bisection auction much less information needs to be revealed to the auctioneer to decide on an allocation and a payment. In the truth-telling equilibrium all valuations in the Vickrey auction and all but the highest valuation in the English auction are revealed to the auctioneer with a precision up to the very last digit. ${ }^{2}$ In the bisection auction through incremental querying the auctioneer asks bidders for very limited information about their valuation, namely whether the valuation is more than an ask price or not.

\footnotetext{
${ }^{1}$ The results of this chapter were first presented in Grigorieva et al. [21].

${ }^{2}$ The reason is that in the version of the English auction that is strategically equivalent to the Vickrey one, all bidders except a bidder with the highest valuation drop out when the price reaches their valuations.
} 
Information already revealed guides the selection of subsequent queries in such a way that only pertinent information is asked. As soon as it becomes clear to the auctioneer that a particular bidder does not possess relevant information, i.e information which helps the auctioneer to find the winner and the price, this bidder is eliminated from the auction. An efficient outcome is determined despite the fact that almost all bidders' valuations have only been partially revealed. Only a bidder with the second highest valuation reveals his valuation, something that is inevitable in a Vickrey implementation (Green and Laffont [20]). All other bidders only reveal their valuation up to a precision required to determine the winner and the precise value of the second highest valuation.

The primal contribution of this chapter is to analyze precisely the communication associated with the revelation of bidders' valuations in these three auctions. The fact that these auctions are strategically equivalent to each other allows us to compare for all three auctions the information bidders are required to reveal about their valuations in equilibrium. Following Kushilevitz and Nisan [35] we measure communication by the number of information bits that bidders should transmit during the auctions in the truth-telling equilibrium. By a bit we mean the smallest unit of information used by a computer, either a 0 or a 1 . We find that in expectation the corresponding number in the bisection auction is far less than in the Vickrey and English auctions. In order to show this we derive formulas for the expected number of information bits in these auctions with $n$ players whose valuations are integer numbers uniformly and independently drawn from the interval $\left[0,2^{R}\right)$ for some positive integer $R$. While we find that in the Vickrey auction $R n$ information bits and in the English auction at least $\frac{1}{3} n 2^{R}$ information bits are to be transmitted, it turns out that the bisection auction requires transmission of at most $2 n+R$ information bits.

\subsection{Communication performance of the bisection auc- tion}

The tool we use to measure communication associated with revelation of information is the amount of data that gets transmitted in equilibrium. Notice that the fact that we have truth telling in equilibrium indeed allows us to identify revelation of information with data transmission. We calculate and compare the expected number of information bits that are to be transmitted by players before the auctioneer can decide upon allocation and payment in the Vickrey, English and bisection auctions. 


\subsubsection{Bounds on data transmission in the bisection auction}

In this subsection we present an implementation of the bisection auction that requires players to transmit at most $2 n+R$ information bits.

The initial ask price is known to all players before the auction starts. During a round with an ask price $p_{r}$ each active player submits a bid 0 or 1 where 0 stands for the statement "My valuation is less than $p_{r}$ " and 1 stands for the contrary. So, during a round each active player sends a single bit of information to the auctioneer. We do not require inactive players to submit bids since the outcome of the auction does not depend on the actions of inactive players. After receiving bids from all active players the auctioneer counts the number of 1's and determines who remains active. To each player that participated in this round the auctioneer sends a message 0 or 1 where 0 stands for the announcement that the bidder is not active anymore and 1 stands for the contrary. A player that remains active uses the information about his own previous action to compute the price of the next round and submits his next bid. Recall that if he said yes in the previous round the price goes up, otherwise the price goes down.

We calculate the expected number of information bits to be transmitted from players to the auctioneer during the auction. We consider the setting where valuations of players are integer numbers drawn uniformly and independently from the interval $\left[0,2^{R}\right)$, for some integer $R$. We assume that each player follows his truthtelling strategy, i.e. he submits 1 to an ask price that is less than or equal to his true valuation and 0 otherwise. From this assumption it follows that in any round an active player submits 1 or 0 with equal probability. Indeed, any active player in current round $r$ has a valuation that is uniformly distributed in the interval between the current lower and the current upper bound. Together with the fact that a current ask price lies in the middle of this interval it gives us the desired probability of $1 / 2$.

Let $E_{\mathrm{B}}(n, R)$ denote the expected number of information bits that will be transmitted from players in the auction of $n$ (remaining) active players and $R$ (remaining) rounds in the case where the winner is not found yet. Similarly, by $E_{\mathrm{B}}^{*}(n, R)$ we denote the expected number of information bits that will be transmitted from players in the auction of $n$ (remaining) active players and $R$ (remaining) rounds in the case the winner has already been found.

First, we find a recursive formula for $E_{\mathrm{B}}(n, R)$. During the first out of $R$ remaining rounds all active players submit bids, so that $n$ information bits are transmitted. Obviously, if only one round remains, then only $n$ bits are transmitted, so 
$E_{\mathrm{B}}(n, 1)=n$. For $R>1$ several situations can occur. If during this round the winner happens to be found, then $n-1$ active players (everyone except the winner) and $R-1$ rounds remain. The probability of this event is equal to $n\left(\frac{1}{2}\right)^{n}$. If during this round the winner is not found then $k$ active players and $R-1$ rounds remain. Notice that if the winner is not found the situation with only one active player in the next round can't occur, so $2 \leq k \leq n$. For $k \neq n$ the probability that $k$ out of $n$ active players say yes is $P(k, n)=\left(\begin{array}{l}n \\ k\end{array}\right)\left(\frac{1}{2}\right)^{n}$. For $k=n$ this probability is $P(n, n)=2\left(\frac{1}{2}\right)^{n}$ (i.e the probability that all $n$ players say yes plus the probability that all $n$ players say no). Thus, for $n>1$ and $R>1$ we find that

$$
E_{\mathrm{B}}(n, R)=n+n\left(\frac{1}{2}\right)^{n} E_{\mathrm{B}}^{*}(n-1, R-1)+\sum_{k=2}^{n} P(k, n) E_{\mathrm{B}}(k, R-1) .
$$

Now we derive a recursive formula for $E_{\mathrm{B}}^{*}(n, R)$. To do that we consider a situation where $n$ active players and $R$ rounds remain in the auction and during a previous round the winner was already found. During the first out of $R$ remaining rounds all active players submit bids, so that $n$ information bits are transmitted during this round. Again, if only one round remains, then only $n$ bits are transmitted, so $E_{\mathrm{B}}^{*}(n, 1)=n$. If only one player happens to be active he remains active till the end of the auction and during the remaining $R$ rounds exactly $R$ bits will be submitted, so $E_{\mathrm{B}}^{*}(1, R)=R$. For $n>1$ and $R>1$ several situations can occur. Depending on the bids in this round $k$ active players $(1 \leq k \leq n)$ and $R-1$ rounds remain. For $k \neq n$ the probability that $k$ out of $n$ players remain active is $P(k, n)=\left(\begin{array}{l}n \\ k\end{array}\right)\left(\frac{1}{2}\right)^{n}$. For $k=n$ this probability is $P(n, n)=2\left(\frac{1}{2}\right)^{n}$. Thus, for $n>1$ and $R>1$ we find that

$$
E_{\mathrm{B}}^{*}(n, R)=n+\sum_{k=1}^{n} P(k, n) E_{\mathrm{B}}^{*}(k, R-1) .
$$

Using these formulas we can compute the expected number of information bits transmitted from the players to the auctioneer in the bisection auction for different combinations of $n$ and $R$. Table 3.1 presents the computational results for $R$ up to 10 and $n$ up to 20 (within an accuracy of 0.001).

We show now that both $E_{\mathrm{B}}^{*}(n, R)$ and $E_{\mathrm{B}}(n, R)$ have upper bounds that are linear in $n$ and $R$.

Lemma 3.2.1. For any $n \in \mathbb{N}$ and $R \in \mathbb{N}, \quad E_{\mathrm{B}}^{*}(n, R) \leq 2 n+R-2$.

Proof. The proof is by induction on $R$. Our induction proposition is

$$
P(R): \quad \text { for every } n \in \mathbb{N}: \quad E_{\mathrm{B}}^{*}(n, R) \leq 2 n+R-2 .
$$




\begin{tabular}{r|rrrrrrrrr}
$n \backslash R$ & 2 & 3 & 4 & 5 & 6 & 7 & 8 & 9 & 10 \\
\hline 2 & 3.500 & 4.750 & 5.875 & 6.938 & 7.969 & 8.984 & 9.992 & 10.996 & 11.998 \\
3 & 5.250 & 6.938 & 8.297 & 9.480 & 10.573 & 11.619 & 12.643 & 13.655 & 14.661 \\
4 & 6.750 & 8.688 & 10.172 & 11.418 & 12.542 & 13.604 & 14.635 & 15.651 & 16.659 \\
5 & 8.125 & 10.273 & 11.873 & 13.179 & 14.333 & 15.411 & 16.450 & 17.469 & 18.479 \\
6 & 9.468 & 11.818 & 13.530 & 14.895 & 16.080 & 17.173 & 18.220 & 19.243 & 20.255 \\
7 & 10.828 & 13.373 & 15.194 & 16.618 & 17.833 & 18.942 & 19.996 & 21.023 & 22.037 \\
8 & 12.218 & 14.954 & 16.882 & 18.364 & 19.610 & 20.734 & 21.796 & 22.827 & 23.843 \\
9 & 13.641 & 16.562 & 18.597 & 20.136 & 21.411 & 22.551 & 23.620 & 24.656 & 25.673 \\
10 & 15.088 & 18.195 & 20.333 & 21.929 & 23.234 & 24.389 & 25.466 & 26.505 & 27.525 \\
11 & 16.554 & 19.847 & 22.086 & 23.739 & 25.073 & 26.243 & 27.328 & 28.371 & 29.393 \\
12 & 18.032 & 21.513 & 23.853 & 25.561 & 26.925 & 28.109 & 29.203 & 30.249 & 31.273 \\
13 & 19.519 & 23.190 & 25.629 & 27.392 & 28.785 & 29.985 & 31.086 & 32.137 & 33.162 \\
14 & 21.011 & 24.877 & 27.413 & 29.230 & 30.653 & 31.868 & 32.976 & 34.031 & 35.058 \\
15 & 22.506 & 26.571 & 29.204 & 31.075 & 32.527 & 33.757 & 34.873 & 35.932 & 36.961 \\
16 & 24.007 & 28.273 & 31.001 & 32.926 & 34.406 & 35.652 & 36.775 & 37.837 & 38.869 \\
17 & 25.502 & 29.980 & 32.804 & 34.781 & 36.291 & 37.551 & 38.682 & 39.748 & 40.781 \\
18 & 27.001 & 31.693 & 34.612 & 36.642 & 38.179 & 39.455 & 40.594 & 41.664 & 42.699 \\
19 & 28.501 & 33.412 & 36.424 & 38.506 & 40.073 & 41.363 & 42.509 & 43.583 & 44.620 \\
20 & 30.000 & 35.134 & 38.242 & 40.375 & 41.970 & 43.275 & 44.429 & 45.506 & 46.545 \\
\hline
\end{tabular}

Table 3.1: The expected number of information bits transmitted from the players to the auctioneer in the bisection auction of $n$ players and $R$ rounds, $E_{B A}(n, R)$.

The basis of induction, $P(1)$, is trivial since $E_{\mathrm{B}}^{*}(n, 1)=n \leq 2 n+1-2=2 n-1$ is true for any $n \in \mathbb{N}$. Now suppose that the proposition $P(R-1)$ is true. Take $n \in \mathbb{N}$. If $n=1$ then we have $E_{\mathrm{B}}^{*}(1, R)=R \leq 2+R-2=R$. If $n>1$ then, using the induction hypothesis,

$$
\begin{aligned}
E_{\mathrm{B}}^{*}(n, R) & =n+\sum_{k=1}^{n} P(k, n) E_{\mathrm{B}}^{*}(k, R-1) \\
& =n+\sum_{k=1}^{n-1}\left(\begin{array}{l}
n \\
k
\end{array}\right)\left(\frac{1}{2}\right)^{n} E_{\mathrm{B}}^{*}(k, R-1)+2\left(\frac{1}{2}\right)^{n} E_{\mathrm{B}}^{*}(n, R-1) \\
& \leq n+\sum_{k=1}^{n-1}\left(\begin{array}{l}
n \\
k
\end{array}\right)\left(\frac{1}{2}\right)^{n}(2 k+R-3)+2\left(\frac{1}{2}\right)^{n}(2 n+R-3) \\
& =n+2 \sum_{k=0}^{n}\left(\begin{array}{l}
n \\
k
\end{array}\right)\left(\frac{1}{2}\right)^{n} k+2 n\left(\frac{1}{2}\right)^{n}+\sum_{k=0}^{n}\left(\begin{array}{l}
n \\
k
\end{array}\right)\left(\frac{1}{2}\right)^{n}(R-3) \\
& =2 n+2 n\left(\frac{1}{2}\right)^{n}+R-3 .
\end{aligned}
$$

Since for any $n$ it holds that $2 n\left(\frac{1}{2}\right)^{n} \leq 1$ we have the desired inequality. 
Theorem 3.2.2. For any integer $n \geq 2$ and $R \in \mathbb{N}, \quad E_{\mathrm{B}}(n, R) \leq 2 n+R$.

Proof. The proof is by induction on $R$. Our induction proposition is

$P(R): \quad$ for every integer $n \geq 2: \quad E_{\mathrm{BA}}(n, R) \leq 2 n+R$.

The basis of induction, $P(1)$, is trivial since $E_{\mathrm{B}}(n, 1)=n \leq 2 n+1$ is true for any integer $n \geq 2$. Now suppose that the proposition $P(R-1)$ is true. Take an integer $n \geq 2$. Using the induction hypothesis and the result of Lemma 3.2.1 we have

$$
\begin{aligned}
E_{\mathrm{B}}(n, R)= & n+n\left(\frac{1}{2}\right)^{n} E_{\mathrm{B}}^{*}(n-1, R-1)+\sum_{k=2}^{n} P(k, n) E_{\mathrm{B}}(k, R-1) \\
= & n+n\left(\frac{1}{2}\right)^{n} E_{\mathrm{B}}^{*}(n-1, R-1)+\sum_{k=2}^{n-1}\left(\begin{array}{l}
n \\
k
\end{array}\right)\left(\frac{1}{2}\right)^{n} E_{\mathrm{B}}(k, R-1)+ \\
& 2\left(\frac{1}{2}\right)^{n} E_{\mathrm{B}}(n, R-1) \\
\leq & n+n\left(\frac{1}{2}\right)^{n}(2 n+R-5)+\sum_{k=2}^{n-1}\left(\begin{array}{l}
n \\
k
\end{array}\right)\left(\frac{1}{2}\right)^{n}(2 k+R-1)+ \\
& 2\left(\frac{1}{2}\right)^{n}(2 n+R-1) \\
= & n+2 n^{2}\left(\frac{1}{2}\right)^{n}+n\left(\frac{1}{2}\right)^{n}(R-5)+2 \sum_{k=2}^{n-1}\left(\begin{array}{l}
n \\
k
\end{array}\right)^{n}\left(\frac{1}{2}\right)^{n} k+ \\
& 4 n\left(\frac{1}{2}\right)^{n}+\sum_{k=2}^{n-1}\left(\begin{array}{l}
n \\
k
\end{array}\right)\left(\frac{1}{2}\right)^{n}(R-1)+2\left(\frac{1}{2}\right)^{n}(R-1) \\
= & n+2 n^{2}\left(\frac{1}{2}\right)^{n}+n\left(\frac{1}{2}\right)^{n}(R-5)+n+R-1-n\left(\frac{1}{2}\right)^{n}(R-1) \\
= & 2 n+R+\left(\frac{1}{2}\right)^{n}\left(2 n^{2}-4 n\right)-1 .
\end{aligned}
$$

It is straightforward to check that $f(n)=\left(\frac{1}{2}\right)^{n}\left(2 n^{2}-4 n\right) \leq 1$ for any integer $n \geq 1$. Thus the desired inequality $E_{\mathrm{B}}(n, R) \leq 2 n+R$ holds.

So we can conclude that during the bisection auction on average not more than $2 n+R$ bits are transmitted from players to the auctioneer. Concerning a lower bound, it is easy to see that during the bisection auction at least $n+R-1$ information bits are to be transmitted from players to the auctioneer. Indeed, during the first round all $n$ active players send a bit and there is at least one active player during the remaining $R-1$ rounds. 


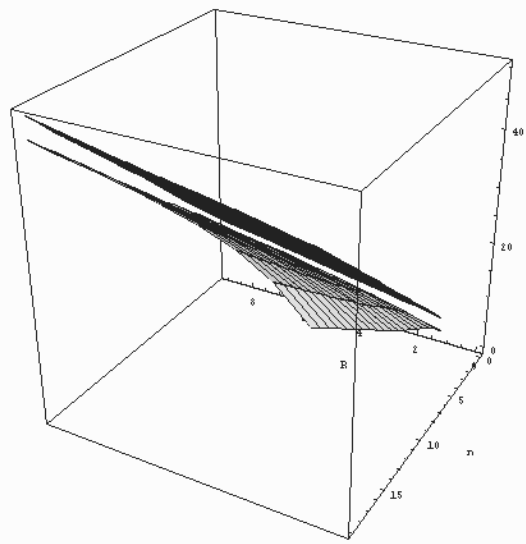

Figure 3.1: The expected number $E_{B A}(n, R)$ of information bits transmitted from players to the auctioneer during the bisection auction, compared to the upper bound $2 n+R$ (view from the back side).

\subsubsection{Comparison with the English auction}

The English auction starts with an ask price equal to 1. From round to round the price increases by a unit increment as long as at least two players announce their willingness to pay. Let us assume that only a synchronization signal is submitted: the auctioneer communicates price increments to all active players by sending 1 , while he communicates the end of the auction by sending 0. Players update their price based on this signal and send 1 to the auctioneer if they are willing to buy at this price and 0 otherwise. ${ }^{3}$

As in the bisection auction, we calculate the expected number of information bits which are to be transmitted during the auction from players to the auctioneer. Suppose that valuations of players are integer numbers uniformly and independently drawn from the interval $[0, L)$ for some integer $L$. We assume that all players follow their truth-telling strategies. In the first round with an ask price equal to 1 the probability that a player says yes is equal to the probability that his valuation is not equal to 0 which is $\frac{L-1}{L}$. For any player $i$ who remains active in the next round it holds that $v_{i} \in[1, L)$. So, the probability of saying yes in the second round (i.e

\footnotetext{
${ }^{3}$ Shoham and Tennenholtz [19] suggest implementation of the English auction where each player communicate only a single bit indicating his willingness to quit the auction. However this implementation requires the use of a global clock whose initial point and rate of change are common knowledge among all participants. We assume that no such a device is available to conduct the auction.
} 
the probability of having valuation not equal to 1) given that the player is active is equal to $\frac{L-2}{L-1}$. And so on.

With $n$ active players in a round there will be $2 \leq k \leq n$ active players in the next round. By $P(k, n, L)$ we denote the probability that exactly $k$ out of $n$ active players, whose valuations are random integer numbers from a half open interval of length $L$, say yes. Thus,

$$
P(k, n, L)=\left(\begin{array}{l}
n \\
k
\end{array}\right)\left(\frac{L-1}{L}\right)^{k}\left(\frac{1}{L}\right)^{n-k} .
$$

Let $E_{\mathrm{E}}(n, L)$ denote the expected number of information bits transmitted from players in the auction of $n$ active players whose valuations are random integer numbers from a half open interval of length $L$. Notice that for $L=2, E_{\mathrm{E}}(n, 2)=n$. For $L \geq 3$ and $n \geq 2$ we find that

$$
\begin{aligned}
E_{\mathrm{E}}(n, L) & =n+\sum_{k=2}^{n} P(k, n, L) E_{\mathrm{E}}(k, L-1) \\
& =n+\sum_{k=2}^{n}\left(\begin{array}{l}
n \\
k
\end{array}\right)\left(\frac{L-1}{L}\right)^{k}\left(\frac{1}{L}\right)^{n-k} E_{\mathrm{E}}(k, L-1) .
\end{aligned}
$$

We will use this recurrent equation to show that the average number of bits $E_{\mathrm{E}}(n, L)$ that will be transmitted from players to the auctioneer has a lower bound of $\frac{1}{3} L n$.

Theorem 3.2.3. For any integer $n \geq 2$ and $L \geq 2, \quad E_{\mathrm{E}}(n, L) \geq \frac{1}{3} L n$.

Proof. The proof is by induction on $L$. Our induction proposition is

$$
P(L): \quad \text { for every integer } n \geq 2: \quad E_{\mathrm{E}}(n, L) \geq \frac{1}{3} L n .
$$

The basis of induction, $P(2)$, is trivial since $E_{\mathrm{E}}(n, 2)=n \geq \frac{1}{3} 2 n$ is true for any integer $n \geq 2$. Now suppose that the proposition $P(L-1)$ is true. Take an integer $n \geq 2$. Using the induction hypothesis we have 


$$
\begin{aligned}
E_{\mathrm{E}}(n, L) & =n+\sum_{k=2}^{n}\left(\begin{array}{l}
n \\
k
\end{array}\right)\left(\frac{L-1}{L}\right)^{k}\left(\frac{1}{L}\right)^{n-k} E_{\mathrm{E}}(k, L-1) \\
& \geq n+\sum_{k=2}^{n}\left(\begin{array}{l}
n \\
k
\end{array}\right)\left(\frac{L-1}{L}\right)^{k}\left(\frac{1}{L}\right)^{n-k} \frac{1}{3}(L-1) k \\
& =n+\frac{1}{3}(L-1)\left[\left(\frac{L-1}{L}\right) n-n\left(\frac{L-1}{L}\right)\left(\frac{1}{L}\right)^{n-1}\right] \\
& =\frac{1}{3} n L+n\left[\frac{2}{3}-\frac{1}{3}\left(\frac{L-1}{L}+\frac{(L-1)^{2}}{L^{n}}\right)\right] .
\end{aligned}
$$

Finally observe that $\frac{L-1}{L} \leq 1$ and $\frac{(L-1)^{2}}{L^{n}} \leq 1$ for any $L$ and any $n \geq 2$.

To compare the communication performance of the bisection and English auctions we look at the same range of valuations for both auctions. So we take interval $\left[0,2^{R}\right)$ and compare $E_{\mathrm{B}}(n, R)$ with $E_{\mathrm{E}}\left(n, 2^{R}\right)$. For valuations uniformly and independently drawn from the interval $\left[0,2^{R}\right)$ the bisection auction requires from players in expectation transmission of at most $2 n+R$ information bits while the English auction requires transmission of at least $\frac{1}{3} 2^{R} n$ bits.

It can easily be checked that for any $R \geq 4$ and any $n$ the upper bound of the expected number of bits transmitted by players in the bisection auction is less than the corresponding lower bound in the English auction. Moreover it can be shown that for all $n$ and $R$ it holds that $E_{\mathrm{B}}(n, R) \leq E_{\mathrm{E}}\left(n, 2^{R}\right)$. From the above bounds it is clear that the expected number of information bits required by the English auction is exponential in the total expected number of information bits required by the bisection auction. Thus we can conclude that the bisection auction requires from players in expectation communication of far less information bits than the English auction.

\subsubsection{Comparison with the Vickrey auction}

To compare the communication performance of the bisection and Vickrey auctions we look again at valuations uniformly and independently drawn from the interval $\left[0,2^{R}\right)$. Any valuation from this interval can be represented using a binary encoding of length $R$. Submission of a valuation expressed in this way consists of $R$ information bits. Since during the Vickrey auction all players submit their exact valuation, this auction of $n$ players requires communication of $R n$ bits.

It can easily be shown that for any $n$ and $R$ the expected number of bits $E_{\mathrm{B}}(n, R)$ transmitted from players in the bisection auction is less than the number $R n$ of 
corresponding bits in the Vickrey auction. Thus, we can conclude that the bisection auction requires from players in expectation far less communication than the Vickrey auction.

\subsection{Conclusion}

While being strategically equivalent to the Vickrey and English auctions the bisection auction outperforms them in terms of information revelation and associated communication. We compared three strategically equivalent auctions and showed that in the truth-telling equilibrium the bisection auction requires the players to transmit less information bits to the auctioneer than the Vickrey and English auctions. 


\section{Chapter 4}

\section{Optimal communication performance}

In this chapter we analyze the communication performance of auctions viewed as algorithms that sequentially queries bidders about their valuations. We investigate and compare the number of queries performed by algorithms until they find the Vickrey outcome. We introduce a measure for comparison that resembles stochastic dominance, but that, to the best of our knowledge, has not been used before for algorithm comparison. With respect to this measure, we prove for the case of two bidders a tight lower bound on the required communication of Vickrey auctions, and show that the bisection auction matches this bound.

\section{$4.1 \quad$ Introduction}

In Chapter 2 we showed that in the proposed bisection auction truth-telling is a weakly-dominant strategy and the equilibrium where all players follow this strategy results in an efficient allocation. The bisection auction shares this property with the Vickrey auction and the English auction. In Chapter 3 we showed that compared to these two auctions the bisection auction needs less communication to determine the allocation and the price for the item. The following natural questions arise: how much communication does the bisection auction need compared to any other auction that possesses the same equilibrium properties, in particularly, does there exist an auction with these equilibrium properties that needs less communication than the bisection auction?

Results of Green and Laffont [20] and Holmström [28] imply that under certain minor restrictions on bidders' utility functions the Vickrey-Clarke-Groves (VCG) 
mechanisms are the only ones that are incentive compatible, have a dominant strategy equilibrium and provide an efficient allocation. Due to this result we restrict our search to this class of mechanisms. For the case of a single indivisible item any mechanism that belongs to this class is a mechanism that finds the Vickrey outcome. The Vickrey outcome requires that the winner is a bidder having the highest valuation and the price he pays for the item is equal to the second-highest valuation.

In this chapter we partially answer the questions posted above, showing that the bisection auction applied to the case of two bidders requires less communication than any other auction that finds the Vickrey outcome. We prove a lower bound for any Vickrey auction ${ }^{1}$, and show that the bisection auction matches this bound. To achieve this goal we view a Vickrey auction, and in particular the bisection auction, as an algorithm that sequentially queries bidders about bits of a binary encoding of their valuations. We investigate and compare the number of queries performed by algorithms until they find the identity of all bidders with the highest valuation and the exact value of the second-highest valuation.

It is widely accepted in theoretical computer science to compare the performance of two algorithms by considering performance of the algorithms in the worst case (see e.g. Knuth [32]). For the problem at hand, worst case analysis hardly makes any sense since for any algorithm there exists a realization of valuations requiring $R n$ queries to provide a Vickrey outcome, where $n$ is the number of bidders and $R$ is the length of the binary encoding of the valuations. Therefore, we need another, more sophisticated tool for algorithm comparison. Given an algorithm we count the number of realizations of valuations for which the number of queries is at most $k$, for some $0 \leq k \leq R n$. We say that algorithm $A^{1}$ is preferred to algorithm $A^{2}$ if for every $k$ the number of realizations of valuations on which algorithm $A^{1}$ finds the Vickrey outcome by performing at most $k$ queries is not less than the same number in algorithm $A^{2}$. For the case of two bidders we show that the bisection algorithm is preferred to any other algorithm that determines the Vickrey outcome. In particular, this result implies that for two bidders having uniformly distributed valuations the bisection algorithm has the lowest average number of performed queries among all Vickrey algorithms.

We conjecture that the bisection algorithm is preferred to any Vickrey algorithm not only in case of two bidders but for any number of bidders. This is left for further research.

\footnotetext{
${ }^{1}$ In this chapter we use term a Vickrey auction in a broad sense to refer to an auction that finds the Vickrey outcome (so not to refer to the seal-bid second price format as we did in the previous chapters).
} 
This result is of interest not only in mechanism design but also in theoretical computer science. Consider the classical problem of comparing two numbers given in binary encoding. The question is which of these numbers is the bigger one? Clearly, a very straightforward algorithm for this problem that is crossing our minds is to compare the first (highest register) digits of the numbers, then the second and so on till a difference is found. The question is whether there exists a more efficient algorithm solving this problem. Despite the fact that this problem is fundamental and our preference measure is natural, to the best of our knowledge, this has not been shown before. Our result can be seen as the proof that the intuitive algorithm above, using in fact binary search, is the preferred algorithm among all algorithms solving the number comparison problem.

\subsection{Vickrey auctions as algorithms}

Suppose there is an auctioneer who wants to sell a single indivisible item to an individual in the set $N=\{1, \ldots, n\}$ of bidders. Each bidder $i$ has a valuation for the item that is given in binary encoding of length $R$. Thus, the valuation of bidder $i$ is a vector $v_{i}=\left(v_{r i}\right), r=1, \ldots, R$, where each element $v_{r i}$ is a binary digit, i.e. is equal to 0 or 1 . The set $V_{i}=\{0,1\}^{R}$ denotes the set of all possible realizations of bidder $i$ 's valuation. A realization of bidders' valuations is a matrix $v=\left(v_{i}\right)_{i \in N}$, where column $i$ corresponds to the valuation of bidder $i$. In the following we call cells in this matrix a box. The set $V=\prod_{i \in N}\{0,1\}^{R}$ is the set of all possible realizations of bidders' valuations. The valuation of a bidder is only known to the bidder itself. In particular, the auctioneer is entirely ignorant concerning the particular realization of valuations. An auction that the auctioneer is employing to retrieve information about bidders' valuations can be viewed as an algorithm that operates on matrices in $V$. To define precisely what we mean by an algorithm, we need some more notation.

For $k \in \mathbb{N}$, a history $h$ of length $k$ is a sequence of $k$ binary digits. So $h=$ $\left(b^{1}, \ldots, b^{k}\right)$, where $b^{j} \in\{0,1\}, j=1, \ldots, k$. The length of the history $h$ is denoted by $\ell(h)$. The initial history is given by $h^{0}=\emptyset$ and has length zero. The history $g$ is a subhistory of $h$, denoted $g \leq h$, if the first $\ell(g)$ digits of $h$ yield the history $g$. The set $C=N \times\{1, \ldots, R\}$ denotes the set of matrix coordinates.

Algorithm. Consider a triplet $A=(H, \varphi, \sigma)$, where $H$ is a collection of histories and $\varphi: H \rightarrow C$, and $\sigma: H \rightarrow\{0,1\}$ are functions. The function $\varphi$ is called the query function, and $\sigma$ is called the stopping criterion. The triplet $A=(H, \varphi, \sigma)$ is an algorithm if:

1. $\emptyset \in H$. 
2. If $h \in H$ and $\sigma(h)=0$, then $(h, 0) \in H$ and $(h, 1) \in H$.

3. If $h \in H$ and $\sigma(h)=1$, then $(h, 0) \notin H$ and $(h, 1) \notin H$.

The interpretation of an algorithm $A=(H, \varphi, \sigma)$ is as follows. First, nature selects an instance $v$ in $V$. The algorithm $A$ starts without any information, represented by the empty vector $h^{0}=\emptyset$. If $\sigma(\emptyset)=1$, the algorithm has solved the problem and stops (of course, stopping at $h^{0}$ is unusual). Otherwise, $\sigma(\emptyset)=0$ and the algorithm opens box $\varphi\left(h^{0}\right)$ of $v$ (this is also referred to as the query performed by the algorithm), which generates the history $h^{1}=\left(v_{\varphi\left(h^{0}\right)}\right)$. If $\sigma\left(h^{1}\right)=1$, the algorithm stops. Otherwise, the algorithm opens box $\varphi\left(h^{1}\right)$, which generates the history $h^{2}=\left(h^{1}, v_{\varphi\left(h^{1}\right)}\right)$. In general, after $k$ steps history $h^{k}$ is generated. If $\sigma\left(h^{k}\right)=1$, the algorithm stops. Otherwise, the algorithm opens box $\varphi\left(h^{k}\right)$, which generates the history $h^{k+1}=\left(h^{k}, v_{\varphi\left(h^{k}\right)}\right)$, and so forth.

Remark. Even though the algorithm only decides on the next query to perform given the announced binary digits, without remembering who announced it, this is of course not a restriction, because the algorithm itself decided to whom the previous question was asked. Thus, to include data regarding the queries itself -not just the answers - into histories will needlessly complicate our notation. It suffices just to remember which answers were given in which order. When necessary we can use the algorithm itself to find out to which question a certain digit was an answer.

Any such algorithm $A$ can be represented by a rooted binary tree with arcs directed from the root to the endnodes. Nodes of this tree correspond to histories in $H$, at which the query $\varphi(h)$ is posed, arcs correspond to answers to the queries $\varphi(h)$. The root of the tree is $h^{0}=\emptyset$. A sequence of nodes on a path from the root to any node of the tree is a realization of algorithm $A$. A path from the root to a node $h$ indicates queries which were performed and answers to them before the algorithm asks the query corresponding to node $h$. Any node $h$ for which the stopping criterion $\sigma(h)=0$ is followed by two arcs. Any node $h$ for which $\sigma(h)=1$ is an endnode. The length of an endnode $e, l(e)$, is equal to the number of nodes (not counting the root) on the path from the root to $h$. Alternatively, $l(e)$ equals the number of arcs on the path from the root to $e$. This length indicates how many queries are performed before algorithm $A$ ends for an instance $v$ for which the algorithm goes along this path. The set of instances in $V$ for which algorithm $A$ goes along this path and ends in node $e$ has cardinality $2^{R n-l(e)}$, since the number of unopened boxes at the moment algorithm $A$ stops is equal to $R n-l(e)$.

Definition 4.2.1. An algorithm $A=(H, \varphi, \sigma)$ is a Vickrey algorithm if the stopping criterion $\sigma$ is defined such that the algorithm stops does not stop before the identity 
of all bidders with the highest valuation ${ }^{2}$ and the exact value of the second-highest valuation are found. A valuation is considered to be the second highest one if there is exactly one strictly higher valuation, or if it is the highest valuation and there are at least two bidders with this valuation.

The bisection algorithm. An example of a Vickrey algorithm is the bisection algorithm, to be denoted by $B$. The bisection algorithm $B$ opens boxes of matrix $v$ in $R$ steps. In step $r$ the algorithm $B$ opens some boxes in the $r \frac{t h}{}$ row of the matrix. Inside a step the boxes can be opened in an arbitrary sequence. To define boxes to be opened in a step we introduce sets $A_{r}$ and $W_{r}$. Initially, $A_{1}=N$ and $W_{1}=\emptyset$.

In step $r$ the bisection algorithm $B$

1. opens box $\{r, i\}$ for all $i \in A_{r}$;

2. defines $Y_{r}=\left\{i \in A_{r} \mid v_{r i}=1\right\}$;

3. defines $A_{r+1}$ and $W_{r+1}$ as follows

- if $\left|Y_{r}\right|=0$ then $W_{r+1}=W_{r}$ and $A_{r+1}=A_{r}$;

- if $\left|Y_{r}\right|=1$ and $W_{r}=\emptyset$ then $W_{r+1}=Y_{r}$ and $A_{r+1}=A_{r} \backslash Y_{r}$;

- if $\left|Y_{r}\right|=1$ and $W_{r} \neq \emptyset$ or if $\left|Y_{r}\right|>1$ then $W_{r+1}=W_{r}$ and $A_{r+1}=Y_{r}$.

After $R$ steps the bisection algorithm finds the Vickrey outcome. The algorithm opens all boxes of all valuations from the set $A_{R+1}$. The valuation of a bidder from this set is a value of the second highest valuation (if the set $A_{R+1}$ contains more than one bidder valuations of all bidders from this set are equal). If $W_{R+1} \neq \emptyset$, it contains the bidder with the highest valuation. Otherwise the set $A_{R+1}$ contains all bidders (at least two) with the highest valuation (that is the case when the highest and the second highest valuations coincide). Note that, the bisection algorithm always finds the identity of all bidders with the highest valuation, the identity of all bidders with the second highest valuation, and the value of the second highest valuation.

Remark. Step $r$ of the bisection algorithm corresponds to round $r$ of the bisection auction. The set $A_{r}$ can be interpreted as the set of players active in round $r$, the set $W_{r}$ as the winner set. The set $Y_{r}$ corresponds to the set of active players who said yes in round $r$. This correspondence is valid because it can be shown that

\footnotetext{
${ }^{2}$ We require an algorithm to find the identity of all bidders with the highest valuation to give them equal chance to get the object in case of ties.
} 
a truth-telling player active in round $r$ of the bisection auction says yes if the $r \underline{\text { th }}$ digit of the binary encoding of his valuation is equal to 1 and says no otherwise. ${ }^{3}$

\subsection{A criterium for communication performance of al- gorithms}

Among all Vickrey algorithms we want to find the one that finds the Vickrey outcome performing as few queries as possible. The typical approach taken when assessing the performance of an algorithm is to make worst-case analysis . In a worst-case analysis of the considered algorithms, an upper bound should be given for the number of queries the algorithm performs for a given number of bidders $n$ and length of valuations $R$. Since there are instances for which any Vickrey algorithm needs to open all $R n$ boxes (e.g. when the valuations of all bidders are equal to each other), the worst case analysis hardly makes any sense to compare the performance of Vickrey algorithms. Here we will use a more sophisticated criterium for optimality of an algorithm than that implied by worst-case analysis.

For each Vickrey algorithm $A$ we denote by $\ell_{A}: V \rightarrow \mathbb{N} \cup\{\infty\}$ the function that assigns to each instance $v$ in $V$ the length of the history after which the algorithm stops (or equivalently the number of queries that algorithm $A$ performs on $v$ ).

Suppose we have two Vickrey algorithms $A^{1}$ and $A^{2}$, and suppose that the designer of algorithm $A^{1}$ challenges the designer of algorithm $A^{2}$ saying: I claim that my algorithm requires less communication than yours on the set $V$ of instances. How could we decide that this is a legitimate claim? We suggest to let designers play the following game. Each contestant starts with an instance bin that initially contains all instances and a discard bin that is initially empty. In the first round of the game $A^{2}$ may choose any instance $v$ from his instance bin. The task of $A^{1}$ is to find an instance $v^{\prime}$ (not necessarily equal to $v$ ) in his instance bin on which his algorithm performs at least as good as algorithm $A^{2}$ does on instance $v$, i.e such that $\ell_{A^{1}}\left(v^{\prime}\right) \leq \ell_{A^{2}}(v)$. If $A^{1}$ is not able to find such an instance, $A^{2}$ beats $A^{1}$ and wins the contest. However, if $A^{1}$ succeeds to find such an instance, both contestants discard the instance they have chosen to their respective discard bins, and the game enters the second round that is played using only the instances that are still left in their instance bins. This procedure is repeated until either $A^{2}$ beats $A^{1}$ at a certain moment, or until the instance bins are empty, in which case we declare $A^{1}$ to be the winner.

\footnotetext{
${ }^{3}$ Actually, the question during the $r^{\underline{t h}}$ round of the bisection auction can be interpreted as "Is the $r^{\underline{t h}}$ digit of your valuation equal to 1 ? "
} 


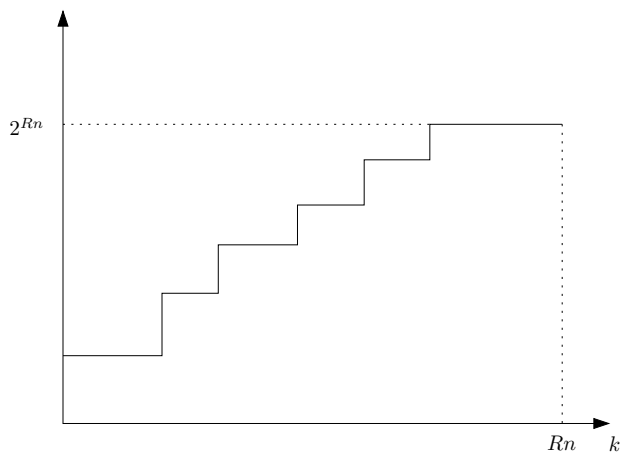

Figure 4.1: Graph of the characteristic function of an algorithm.

Actually it is fairly easy to find optimal strategies for both designers. Designer $A^{2}$ can basically chose any instance from his instance bin while designer $A^{1}$ should, when faced with instance $v$, select an instance $v^{\prime}$ that has the highest value of $\ell_{A^{1}}$ among those instances that are still left in his instance bin and for which $\ell_{A^{1}}\left(v^{\prime}\right) \leq \ell_{A^{2}}(v)$ still holds. Notice that following the optimal strategy designer $A^{1}$ wins the game if for all $k$ the number of instances for which algorithm $A^{1}$ performs at most $k$ queries is not less than the number of instances for which algorithm $A^{2}$ performs at most $k$ queries. Using this observation we can state our criterium for algorithm comparison in a more formal way.

Definition 4.3.1. The characteristic function of an algorithm $A$ is the function $F_{A}(k)$ that counts the number of instances in $V$ for which $\ell_{A}(v) \leq k$.

Notice that the characteristic function of an algorithm enables us to identify the number of instances for which the algorithm terminates in exactly $k$ steps as $F_{A}(k)-F_{A}(k-1)$.

Using the tree representation of algorithm $A$ we can say that

$$
F_{A}(k)=\sum_{j=1}^{k}\left|E_{j}\right| 2^{R n-j},
$$

where $E_{j}$ is the set of endnodes with length $j$. Figure 4.1 shows an example of a graph that corresponds to the characteristic function of an algorithm.

Definition 4.3.2. An algorithm $A^{1}$ is preferred to an algorithm $A^{2}, A^{1} \succeq A^{2}$, if $F_{A^{1}}(k) \geq F_{A^{2}}(k)$ for all $k$. 
Notice that if an algorithm $A^{1}$ is preferred to an algorithm $A^{2}$ then the graph of $F_{A^{1}}(k)$ is never below the graph of $F_{A^{2}}(k)$.

Actually Definition 4.3.2 resembles the definition of first-order stochastic dominance (see, e.g. Levy [38]) if we assume the uniform distribution of instances. Indeed, for the case at hands, it would state that an algorithm $A^{1}$ first-order stochastically dominates an algorithm $A^{2}$ if for every number of queries $k$, the probability of stopping after at most $k$ queries is not smaller for $A^{2}$ than for $A^{1}$. Indeed, rescaling the characteristic function along the $y$-axis (dividing by the total number of instances $2^{R n}$ ) we get the function that, for every number of queries, shows the probability that on a randomly chosen instance the algorithm performs at most as many queries.

Definition 4.3.3. Given a class of algorithms, an algorithm $A^{1}$ is an optimal algorithm if $A^{1} \succeq A$ for all algorithms $A$ in this class.

Not all algorithms can be considered as candidates to be an optimal algorithm. In the following we show that a cyclic algorithm can not be optimal.

Definition 4.3.4. An algorithm $A=(H, \varphi, \sigma)$ is cyclic if there are histories $g, h \in H$ with $g \leq h$ such that $\varphi(g)=\varphi(h)$. Consequently, an algorithm $A=(H, \varphi, \sigma)$ is acyclic if for all histories $g, h \in H$ with $g \leq h$ holds $\varphi(g) \neq \varphi(h)$.

An acyclic algorithm never performs the same query, i.e. never asks a bidder to report a specific digit of his valuation more than once. Therefore an acyclic algorithm always ends within $R n$ performed queries.

Proposition 4.3.5. For any cyclic Vickrey algorithm $A^{1}=\left(H^{1}, \varphi^{1}, \sigma^{1}\right)$ there is a Vickrey algorithm $A^{2}=\left(H^{2}, \varphi^{2}, \sigma^{2}\right)$ such that $F_{A^{2}}(k) \geq F_{A^{1}}(k)$ with strict inequality for some $k$.

Proof. Let us construct such an algorithm $A^{2}$. Consider histories $a, b, c \in H^{1}$ such that $\sigma^{1}(c)=1, a \leq c, b \leq c, a \leq b$ and $\varphi^{1}(a)=\varphi^{1}(b)$. Since algorithm $A^{1}$ is cyclic such histories exist. We construct algorithm $A^{2}$ in the following way. Algorithm $A^{2}$ performs the same queries as algorithm $A^{1}$ does, except on instances that generate history $b$. On these instances it skips query $\varphi^{1}(b)$ and goes on asking the same queries as $A^{1}$ does after query $\varphi^{1}(b)$. By skipping query $\varphi^{1}(b)$ algorithm $A^{2}$ does not lose any essential information and thus finds the Vickrey outcome based on the same information as algorithm $A^{1}$ has. Obviously, on the instances that generate history $b$ algorithm $A^{2}$ stops after $l(c)-1$ queries (i.e. one query less than algorithm $A^{1}$ ) while on other instances it performs the same number of queries as $A^{1}$ does. Thus, in comparison to algorithm $A^{1}$, algorithm $A^{2}$ has more instances for which 
it performs $l(c)-1$ queries and less instances for which it performs $l(c)$ queries. It means that $F_{A^{2}}(l(c)-1)>F_{A^{1}}(l(c)-1)$ while $F_{A^{2}}(k)=F_{A^{1}}(k)$ for all other $k$.

This result implies that a cyclic algorithm cannot be an optimal one. Thus, when searching for an optimal algorithm, we may restrict ourselves to studying acyclic algorithms.

\subsection{Optimal communication performance of the bisec- tion algorithm in case of two bidders}

Here we consider the case where there are only two bidders, i.e. $N=\{1,2\}$. We show that in this case the bisection algorithm is an optimal one among all Vickrey algorithms. Let

$$
v=\left[\begin{array}{cc}
a_{1} & b_{1} \\
\vdots & \vdots \\
a_{R} & b_{R}
\end{array}\right] .
$$

Definition 4.4.1. A matrix row $\left(a_{r}, b_{r}\right)$ for some $r$ is called a double if $a_{r}=b_{r}$ and is called a single otherwise.

Let $p_{A}: V \rightarrow \mathbb{N}$ be the function that assigns to each instance $v$ in $V$ the number of rows that algorithm $A$ opens in $v$ before it stops.

Let $g_{A}(k)$ be the function that defines the number of instances in $V$ for which $p_{A}(v) \leq k$.

Since a Vickrey algorithm $A$ should find the exact value of the second-highest valuation (and thus to open all its boxes) the number of queries that $A$ performs on an instance $v$ is equal to the number of rows that $A$ opens in $V$ before it stops plus $\mathrm{R}$ :

$$
\ell_{A}(v)=p_{A}(v)+R
$$

It means that for any two Vickrey algorithms $A^{1}$ and $A^{2} g_{A^{1}}(k) \geq g_{A^{2}}(k)$ for all $k$ implies that $F_{A^{1}}(k) \geq F_{A^{2}}(k)$ for all $k$. So, to prove that algorithm $A^{1}$ is preferred to algorithm $A^{2}$ it is enough to show that $g_{A^{1}}(k) \geq g_{A^{2}}(k)$ holds for all $k$.

Proposition 4.4.2. For an instance $v$ which contains $R$ doubles any Vickrey algorithm, before it stops, opens all rows. For any other instance any Vickrey algorithm opens at least one single. 
Proof. A Vickrey algorithm as defined in Definition 4.2.1 finds the identity of all bidders with the highest valuation. To do that it should be verified that either valuations of both bidders equal to each other (and thus they both have the highest valuation) or valuations are different. The former can be only shown by opening all boxes, the latter by opening at least one row with unequal entries.

Remark. If we do not require an algorithm to find all bidders with the highest valuation then the statement of Proposition 4.4.2 does not hold. Consider the following example. Suppose an algorithm has opened

$$
\left[\begin{array}{ll}
0 & 0 \\
1 & 1 \\
0 & *
\end{array}\right]
$$

after five queries. The revealed information is enough to find the second highest valuation (which is equal to the valuation of bidder 1) as well as the identity of a bidder with the highest valuation (that is bidder 2). So if we are satisfied with finding only a bidder with the highest valuation we can stop without opening the remaining box. That means that the algorithm, before it stops, may neither open all $R$ rows nor a single.

Lemma 4.4.3. For every Vickrey algorithm A applied to the case of two bidders

$$
g_{A}(k) \leq \sum_{j=1}^{k} 2^{2 R-j} .
$$

Proof. Let us consider an algorithm $\bar{A}$ that does the same as algorithm $A$ but stops as soon as the first single is opened. Let the set of instances $M$ be equal to $V$ and $M_{e}$ and $M_{u}$ be empty sets. Go through the following procedure. Take an instance $v$ from $M$. Consider the step in algorithm $\bar{A}$ where the first row ${ }^{4}$ has been opened. W.l.o.g. in this step box $b_{r}$ has been opened. Define instance $v^{\prime}$ as follows:

$$
\begin{array}{ll}
a_{j}^{\prime}=a_{j} & \text { for all } j \\
b_{j}^{\prime}=b_{j} & \text { for all } j \neq r \\
b_{r}^{\prime} \neq b_{r} &
\end{array}
$$

Obviously, instance $v^{\prime}$ is also from $M$.

\footnotetext{
${ }^{4}$ By "the first row" we do not refer to the upper row of matrix $v$ but to a row that has been opened first when no other row is completely opened yet. The same holds for the usage of "the second row".
} 
Clearly, either $a_{r}=b_{r}$ or $a_{r}^{\prime}=b_{r}^{\prime}$. If $a_{r}=b_{r}$ then update

$$
\begin{aligned}
& M_{e}:=M_{e} \cup\{v\} \\
& M_{u}:=M_{u} \cup\left\{v^{\prime}\right\} .
\end{aligned}
$$

Otherwise

$$
\begin{aligned}
& M_{e}:=M_{e} \cup\left\{v^{\prime}\right\} \\
& M_{u}:=M_{u} \cup\{v\} .
\end{aligned}
$$

In both cases $M:=M \backslash\left\{v, v^{\prime}\right\}$.

Repeat the procedure from the beginning until set $M$ is empty. It is clear from the construction that when the procedure ends we have $\left|M_{e}\right|=\left|M_{u}\right|=2^{2 R-1}$. Obviously for any instance from $M_{u}$ algorithm $\bar{A}$ stops after the first row is opened, since this row is a single.

Let $V_{k}(A) \subseteq V$ be the set of instances for which algorithm $A$, before it stops, opens $k$ rows. Then $V_{1}(\bar{A})=M_{u}$ and $\left|V_{1}(\bar{A})\right|=2^{2 R-1}$.

Using Proposition 4.4.2 we know that algorithm $A$ stops not earlier than algo$\operatorname{rithm} \bar{A}$ and thus $V_{1}(A) \subseteq V_{1}(\bar{A})$. That implies $g_{A}(1)=\left|V_{1}(A)\right| \leq 2^{2 R-1}$.

Now apply the same procedure to the set $M_{e}$ by considering the step in the algorithm $\bar{A}$ where the second row is opened. Note that for any instance from $M_{e}$ the first opened row is a double. At the end of the procedure we have the set $V_{2}(\bar{A})$, the set of instances for which algorithm $\bar{A}$ stops after the second row is opened. The cardinality of this set is $2^{2 R-2}$. Again, by Proposition 4.4.2, algorithm $A$ stops not earlier than algorithm $\bar{A}$ and thus $V_{1}(A) \cup V_{2}(A) \subseteq V_{1}(\bar{A}) \cup V_{2}(\bar{A})$, which implies that $g_{A}(2)=\left|V_{1}(A) \cup V_{2}(A)\right| \leq 2^{2 R-1}+2^{2 R-2}$.

Iterating this procedure we can show that for any $k \leq n$ it holds that $\left|V_{k}(\bar{A})\right|=$ $2^{2 R-k}$. Since

$$
\bigcup_{j=1}^{k} V_{j}(A) \subseteq \bigcup_{j=1}^{k} V_{j}(\bar{A})
$$

we get the desired result

$$
g_{A}(k)=\left|\bigcup_{j=1}^{k} V_{j}(A)\right| \leq \sum_{j=1}^{k} 2^{2 R-j}
$$

The bisection algorithm $B$ applied to $(R \times 2)$-matrix $v$ opens rows in the topdown fashion as long as no single is opened. As soon as the first single is opened the 
algorithm is able to determine the identity of the highest valuation (and at the same time the identity of the second highest). Then the algorithm opens the remaining boxes of the second highest valuation.

Theorem 4.4.4. For the case of two bidders the bisection algorithm $B$ is an optimal algorithm among all Vickrey algorithms.

Proof. First of all, notice that after the algorithm $B$ finds the first single no new row is opened. It means that $V_{k}(B)=V_{k}(\bar{B})$. From the proof of Lemma 4.4.3 it follows that $\left|V_{k}(B)\right|=2^{2 R-k}$ and for any $k \leq n$

$$
g_{B}(k)=\sum_{j=1}^{k} 2^{2 R-j} .
$$

Using the result of Lemma 4.4.3, for any Vickrey algorithm $A$ and any $k$ we get

$$
g_{A}(k) \leq g_{B}(k)
$$

and consequently

$$
F_{A}(k) \leq F_{B}(k)
$$

Corollary 4.4.5. For the case of two bidders with uniformly distributed valuations the bisection algorithm has the lowest expected communication among all Vickrey algorithms.

\subsection{Conclusion}

In this chapter we have shown that for the case of two bidders the bisection algorithm has the optimal communication performance in the class of Vickrey algorithms. An interesting and very natural question is to extend this result to an arbitrary number of bidders. Despite obvious similarities, the problem with multiple bidders seems to be much more difficult to tackle than the problem with only two bidders. At least, the presented approach is not straightforwardly extendable to handle the general problem. We leave this question as a conjecture for further research.

Conjecture 4.5.1. The bisection algorithm $B$ applied to the case with an arbitrary number of bidders is an optimal algorithm among all Vickrey algorithms. 


\section{Chapter 5}

\section{An experimental investigation of bidding behavior in the bisection auction}

We present a laboratory experiment in which we investigate bidding behavior in the proposed bisection auction, and compare it with two classical auction formats - the Vickrey auction and the English auction. We test whether subjects behave strategically equivalent, following the dominant truth-telling strategy, as predicted by theory. Furthermore, we provide some insights concerning the learning process, the efficiency of allocation, and the revenue to the auctioneer. The data show that the bisection auction performs better than the Vickrey auction and only in some terms worse than the English auction. ${ }^{1}$

\section{$5.1 \quad$ Introduction}

In Chapter 2 it is shown that the bisection auction is strategically equivalent to the Vickrey auction (and hence also to the English auction). Therefore it preserves efficiency and has truth-telling as a dominant strategy. While being strategically equivalent to the Vickrey and English auctions the bisection auction outperforms them in terms of speed and valuation revelation requirements. Contrary to the English auction the bisection procedure guarantees a fast termination of the proposed auction. In comparison with the Vickrey auction, the bisection auction requires much less information to be revealed to the auctioneer. Through incremental querying the auctioneer asks bidders for very limited information about their valuation, namely

\footnotetext{
${ }^{1}$ The results of this chapter were first presented in Grigorieva and Strobel [25].
} 
whether the valuation is more than an ask price or not. Information already revealed guides the selection of subsequent queries in such a way that only pertinent information is asked. As soon as it becomes clear to the auctioneer that a particular bidder does not possess relevant information, i.e information which helps the auctioneer to find the winner and the price, this bidder is eliminated from the auction. An optimal outcome is determined despite the fact that almost all bidders' valuations have only been partially revealed. Only the bidder with the second highest valuation reveals it entirely, something that is inevitable in a Vickrey implementation (see Green and Laffont [20]).

Given the advantages of the bisection auction, it seems strange that one does not come across it in practical applications. One potential reason is that while all three auctions are theoretically equivalent, they might differ substantially from a behavioral point of view when boundedly rational decision makers are involved. Several experiments were conducted so far for testing the strategic equivalence of the Vickrey and English auctions in the laboratory. The results by and large show that in contrast with theory players typically do not behave in an equivalent way. Behavior according to the dominant truth-telling strategy was observed more often in the English auction (see e.g. Coppinger et al. [14], Kagel et al. [30]). In the Vickrey auction, observed bids often exceeded the predicted level (see e.g. Harstad [27], Kagel et al. [30] and Kagel and Levin [31]). ${ }^{2}$ As conjectured in Kagel [29], this difference in behavior can be attributed to different information flows inherent in the structure of the two auctions. The structure of the English auction makes it relatively transparent to players that they should not bid above their valuations - any time a player bids above his valuation and wins he necessarily loses money. In contrast, in the Vickrey auction there is no such direct link between the bid of a winner and the price he has to pay. In the Vickrey auction a player does not necessarily lose money if he bids above his private valuation and wins. This sustains the illusion that overbidding improves the probability of winning with only the little risk of paying a price above the own valuation. The feedback mechanism to learn and to correct overbidding is therefore weaker in the Vickrey procedure.

The reported breakdown of the strategic equivalence of the Vickrey and English auctions with respect to behavior raises justified doubts on the behavioral equivalence of the bisection auction and the others. The bisection auction due to its more complicated rules seems to be more difficult to understand. A particularly uncommon feature for auctions is that the price might be moving up and down. It

\footnotetext{
${ }^{2}$ Some papers, e.g. Cox et al. [15] and Smith [57], report the Vickrey auctions in which prices converge to the dominant strategy price from below. However, in these experiments subjects were explicitly prohibited from bidding above their valuations.
} 
might make bidders think that with bidding high initially and hoping that the price would go down later in the auction, they can increase their profit by overbidding. In this sense the bisection auction also lacks the tight cognitive connection between the probability to win and the price to be paid. The situation calls for an initial test of behavioral equivalence before putting the bisection mechanism to practice. One could argue, that market forces will drive out irrational behavior and will lead to the dominant strategy behavior sooner or later. The process, however, will not be free of frictions (e.g. law suits) and thus a good ex-ante estimate of the behavioral anomalies in the bisection auction will be helpful. The goal of this chapter is to make some first steps into the investigation of the practical usage of the bisection auction. Therefore we test all three auction types in a laboratory experiment and compare them with respect to truth-bidding, revenue and efficiency.

In comparison to the other auctions the bisection auction did fairly well and much better than we initially expected. The performance is quite comparable to the English auction and in terms of some measurements better than the Vickrey auction.

The chapter is organized as follows. Section 5.2 describes the experimental design and laboratory procedure. Section 5.3 reports the results and tests and Section 5.4 concludes with a summary and some open questions. In the Appendices 5.5 - 5.7 the instructions, the post-experimental questionnaire and additional tables with relevant data are presented.

\subsection{Experimental design and procedure}

To compare the auction mechanisms we designed an experiment with three different treatments VA, EA and BA which correspond to the Vickrey, English and bisection auction, respectively. The experiment was computerized with the help of zTree (Fischbacher [18]). Throughout the experiment we used ECU (Experimental Currency Unit) as a fictitious currency for expressing prices, values, bids and earnings. One session lasted for 10 periods and was played by 9 subjects. In each period they were randomly partitioned into three groups of three bidders. At the beginning of each period every bidder got to know privately her value which was independently drawn from the uniform distribution over the interval $[32,96) .{ }^{3}$ For every session of one treatment a new set of valuations and a new matching scheme were randomly generated. However, in order to maximize comparability of treatments we used the same sets of valuations and the same matching schemes across treatments. After

\footnotetext{
${ }^{3}$ This probability function was publicly known.
} 
learning their value subjects had to bid against the other group members in order to win the auction. The auction mechanism depended on the treatment.

Vickrey auction (VA): Subjects were asked to submit one bid which had to be an integer number from the interval $[0,128)$. The subject with the highest bid won the auction and received the object (i.e. she got her private value). The price the winner had to pay was equal to the second-highest bid that was made within the group.

English auction (EA): The English auction was implemented by using an ascending clock procedure. The price started at zero and increased continuously at a rate of 1 ECU per 2 seconds with the maximum possible price of 127 . Subjects had drop out at the price they were not willing to bid anymore. Dropping out was irrevocable so that a bidder could no longer bid on the object. The last bidder of the group won the object at the price 1 ECU below the price where the next-to-last bidder dropped out. ${ }^{4}$

Bisection auction (BA): The initial interval for the bisection auction was $[0,128)$. The initial ask price was set to 64 . The winner and the price were then determined by the procedure described in section 2.2 .

In all treatments ties could happen if at least two persons show exactly the same behavior and end up to be the "highest" bidders. In such cases the winner was randomly determined among the tied bidders. At the end of each period the winner of the auction received his private value but had to pay the determined final auction price. Each of the bidders was informed whether or not she bought the object, the selling price of the object, as well as her gains and losses.

The experiment was conducted in the experimental behavior laboratory of the economic faculty at Maastricht University. In total 135 subjects took place in the experiment. Most of them were undergraduate students from economics or business. They could register for the experiment via Internet via their student id. By this we prevented double registration. In total we conducted 5 sessions for each treatment, with 9 subjects each. When subjects arrived at the laboratory they were randomly

\footnotetext{
${ }^{4}$ We explicitly have chosen this instead of the classical version of the ascending clock English procedure where the winner gets the object at the price when the next-to-last bidder dropped out. This choice allowed us to simplify the part of the subjects instructions where we explained the moment of dropping out, namely "... at the price you are not willing to bid any more". This change in rules has the consequence that in the implemented version dropping out as soon as the price exceeded the private value is the dominant truth-telling strategy. Apart from that, these two versions are identical and either of them can be used for comparison with the bisection and Vickrey procedures.
} 
placed at isolated computer terminals, so that they neither could see screens of the others nor get into eye contact with them. The sessions lasted for about 60 minutes.

Before the start of a session subjects received written instructions (see Appendix 1). They were allowed to privately ask questions which were also privately answered by the experimenters. As a part of the instructions subjects were provided with examples of players' valuations and were asked to calculate the profit of each player for some assumed outcomes. The calculations were privately checked. The experiment started only when every calculation was done correctly. After a session we asked participants to fill in a post-experimental questionnaire (see Appendix 2) where they could explain how they made their decisions and give any comments on the experiment.

Each participant received an initial endowment of 3 EURO. At the end of the experiment the amount which was earned was converted from ECU into Euro at the rate of $1 \mathrm{EURO}=4.5 \mathrm{ECU}$. For the case that the earned amount was negative, the losses were deducted from the initial endowment. Average earnings of the participants were about 13 Euro.

\subsection{Experimental results}

By and large we were able to replicate the common results on the Vickrey and the English auction. ${ }^{5}$ The data further shows that the bisection auction does not perform worse than the Vickrey auction. In some aspects it was doing better and matches the performance of the English auction. In the following we will analyze the data in more detail with respect to truth-telling, learning, auctioneer's revenue and efficiency. If not mentioned explicitly we will base our statistical tests on the session level (i.e. on statistically independent observations). Therefore we aggregate the data by averaging over all observations within a session.

\subsubsection{Bidding behavior}

Theory predicts bidders in all auctions types to follow a truth-telling strategy. In the following we check whether this is indeed the case or whether subjects tend to underor overbid. When classifying subjects as truth-tellers we accept small deviations from the private value and call it a value bid. Reasons for small deviations could be that subjects ignored the possibility of ties. In such cases small deviations (i.e. by 1 ECU) from true-value bidding do not change the subjective expected payoff. Another reason for dropping out a bit too early or too late in the English auction

\footnotetext{
${ }^{5}$ See also our discussion in the introduction to this chapter.
} 
could be the fear of missing the exact time to drop out or indeed missing it. In the following paragraphs we define value bids in detail and report results for the different treatments.

Vickrey auction: For VA truth-telling means to bid exactly the private value $v_{i}$, hence we consider any bid in the set $\left\{v_{i}-1, v_{i}, v_{i}+1\right\}$ as a value bid. If the bid was above or below this set we consider it as over- or underbidding, respectively.

The data shows that $48 \%$ of all 450 Vickrey bids are value bids while $30 \%$ of the bids were too high and $22 \%$ of the bids were too low. Five subjects out of 45 followed the value bidding strategy in all 10 auction periods. The average number of periods where a subject bid his value was 3.6 periods.

English auction: In the implemented version of the English auction the truthtelling strategy is to drop out as soon as the price $p$ exceeds the private valuation $v_{i}$, i.e. if $p=v_{i}+1$. Thus we consider it a value bid if the drop-out price was in the set $\left\{v_{i}, v_{i}+1, v_{i}+2\right\}$. If a player dropped out at a price below or above this set, we considered it as under- and overbidding, respectively. Normally one particular player, the winner of the auction, does not drop out and therefore does not completely reveal his bidding strategy. These cases we regarded as value bids as long as the elicited behavior was consistent with value bidding. ${ }^{6}$

Among 450 bids in the EA treatment $75 \%$ were value bids, $16 \%$ subjects were underbids and $9 \%$ overbids. If we neglect the observations from the auction winners (because their willingness to bid was not fully revealed), we observe a higher frequency of non-value bids (i.e. $63 \%$ value bids, $24 \%$ underbids and $13 \%$ overbids). Nine subjects (or $20 \%$ of all subjects) are consistent with value bidding in all 10 auction periods. The average number of periods where a subject bid his value was 7.4 periods.

Bisection auction: The bisection auction was implemented along the description in Section 2.2. Each period consisted of 7 rounds. In each round active bidders have to submit either yes or no for a current ask price. The truth-telling strategy is to regard the private valuation as threshold value and to state yes if the ask price is less or equal to the threshold, and no otherwise. It is an inherent feature of the bisection auction that bidders thresholds are only partially revealed. Hence, we cannot analyze behavior precisely but we have to rely on bidder's observable actions. Thus, we consider a strategy as value-bidding if in all observed rounds the bidder's submissions are consistent with a threshold from the set $\left\{v_{i}-1, v_{i}, v_{i}+1\right\}$.

\footnotetext{
${ }^{6}$ This classification can be viewed as too optimistic, i.e. we assume that buyers with a private value above the auction price would never overbid. However, we will also report data without taking these buyers into account.
} 
Table 5.1: Value bids across different treatments.

\begin{tabular}{lccc}
\hline & VA & EA & BA \\
\hline all observations & 450 & 450 & 450 \\
value bids & $48 \%$ & $75 \%$ & $72 \%$ \\
underbids & $22 \%$ & $16 \%$ & $14 \%$ \\
overbids & $30 \%$ & $9 \%$ & $14 \%$ \\
\hline fully informative observations & 450 & 305 & 164 \\
value bids & $48 \%$ & $63 \%$ & $68 \%$ \\
underbids & $22 \%$ & $24 \%$ & $17 \%$ \\
overbids & $30 \%$ & $13 \%$ & $15 \%$ \\
\hline number of value bidders & 5 & 9 & 7 \\
average number of truth-telling periods per subject & 3.6 & 7.4 & 6.7 \\
\hline
\end{tabular}

If the latent threshold proved to be lower or higher than this set, then the bidding strategy was considered to be an under- or overbid, respectively. ${ }^{7,8}$

The analysis of the data gives us the following results: in $72 \%$ of all 450 cases subjects were consistent with value bidding, in 14\% they were underbidding and in $14 \%$ they overbid. If we restrict the analysis to those bidders who were active all 7 rounds of a period (i.e subjects whose strategy we could observe to the very end) the frequencies were almost the same: $68 \%$ value-bidding, $17 \%$ underbidding and $15 \%$ of overbidding. Seven subjects out of 45 (or 15\%) followed the value bidding strategy in all 10 auction periods. The average number of periods where a subject was consistent with value-bidding was 6.7 periods.

Table 5.1 gives an overview over the value bids across treatments. It seems evident that $\mathrm{EA}$ and $\mathrm{BA}$ outperform $\mathrm{VA}$ with respect to value bidding. In order to test this we calculated the average frequency of value bidding per session and treatment, using all observations as well as using only the fully informative observations (i.e bids where we could observe the strategy to the very end). A nonparametric Mann-Whitney-U test on the session level yields a significant difference between BA and VA as well as between EA and VA. In both cases the null hypothesis that

\footnotetext{
${ }^{7}$ As for the English auction this classification can be seen as too optimistic. Hence we also report data from those bidders who stayed active till the end of the auction (at least one bidder per auction).

${ }^{8}$ Notice that in a period a player can't overbid in one round and underbid in another. Indeed if in a round a player overbids and stays active, then in the next rounds the ask prices are certainly above his valuation so that underbidding is not possible anymore. Similar, if in a round a player underbids and stays active, then in the next rounds the ask prices are certainly below his valuation so that overbidding is not possible anymore.
} 

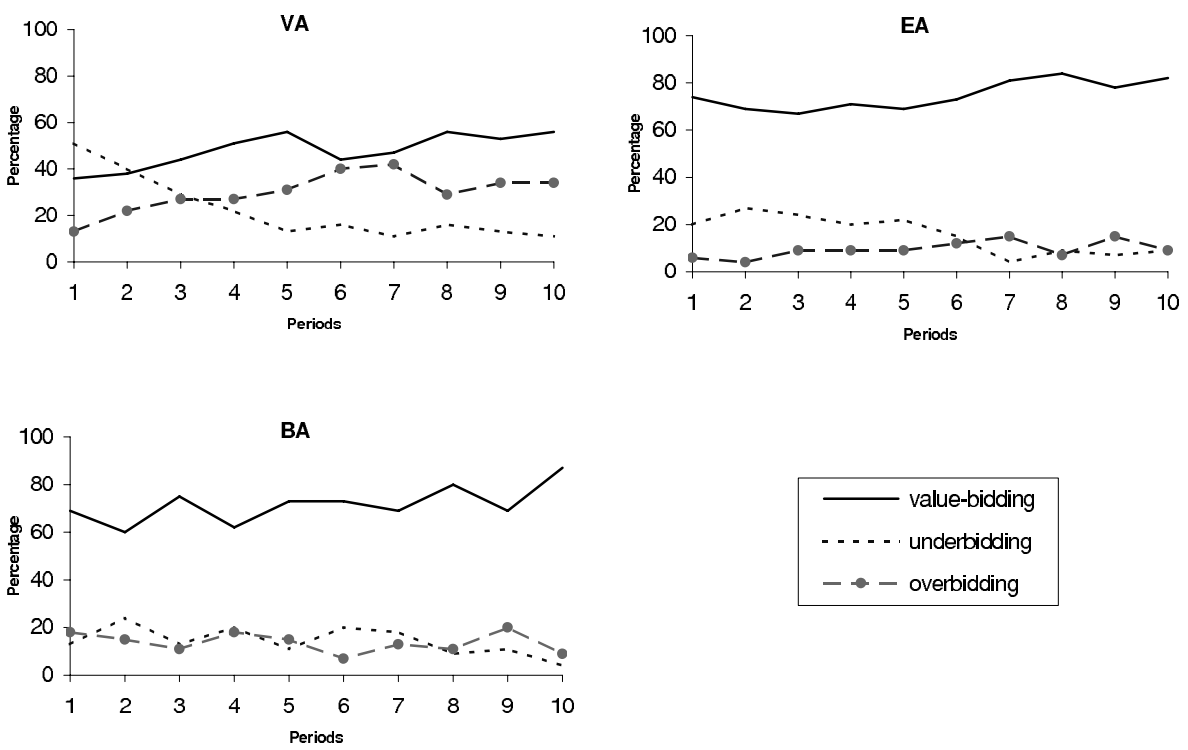

Figure 5.1: Bidding behavior: frequency of the different types of bids.

the frequency of value bidding is equal was rejected in favor of statement that the frequency is smaller in VA treatment (Mann-Whitney-U test, $N=10$ for each comparison, one-sided; based on all observations: $p=0.004$ for both comparisons; based on the fully informative observations: $p=0.079$ for comparison VA with BA and $p=0.0159$ for comparison VA with EA). Between treatments BA and EA we did not find any significant difference. We summarize this in

Result 1. In terms of value bidding the bisection auction is comparable to the English auction and significantly better than the Vickrey auction.

\subsubsection{Learning process}

In section 5.3.1 we analyzed the different auction formats from a static viewpoint. However for the question of practical usability of the bisection auction it is also important to take a dynamic viewpoint. Do subjects learn to place value bids? To answer this we analyze whether subjects get closer to value-bidding as they gained more experience. For a first glance, Figure 5.1 shows how the frequencies of valuebidding, under- and over-bidding develop over time.

For each of the treatments, there is a tendency that value-bidding increases 
over time and underbidding decreases over time. For VA these tendencies are most pronounced. With respect to overbids only VA shows a clear tendency, with the other treatments it seems not to be clear. Interestingly, the number of overbids in VA tends to increase rather than to decrease. In order to test the results statistically we compare the number of deviations from value-bidding in the beginning of a session (periods 1-3) with the number of deviations at the end of a session (periods 8-10) for each subject. We assume one subject to be an independent observation and test the following null hypothesis: the number of deviations from value-bidding in periods 1-3 is the same as in periods 8-10. For each the treatments we can reject the null hypothesis in favor of the alternative hypothesis that the number of deviations in the beginning of a session is higher than at the end of a session (Wilcoxon signed-rank test, $N=45$ for each treatment, $p \leq 0.02$, one-sided).

The assumption of one subject being an independent observation is disputable. It rests on the fact that subjects were randomly matched to other subjects in each round. They neither learned to whom they were matched nor did they learn something about the other bidders values or bidding strategies. The only thing they got to know was the final price and whether they bought the object or not. ${ }^{9}$ Nevertheless, subjects interacted with each other repeatedly during 10 periods of a session (though without knowing against whom they played) and therefore their behavior could be considered as not strictly independent. Hence, we run the same test on the level of independent observations (session-level) and we get similar results, although only weakly significant (Wilcoxon signed-rank test, $N=5$ for each treatment, $p=0.0625$, one-sided).

The tests above only make use of the cases were indeed some differences were found in the behavior at the beginning and at the end (see e.g. Siegel and Castellan [55]). This applies to 23, 29, and 26 subjects for the treatments VA, EA, and BA, respectively. Among those subjects who did not contribute to the test results we can distinguish between subjects who did not deviate from value bidding in any of the periods 1-3 and 8-10 and those subjects who did deviate to the same extend in the beginning and the end.

The first group of subjects always behaved rationally and therefore could not improve behavior. The numbers of subjects were 5, 12, and 15, for VA, EA, and BA, respectively. Apparently there is more rational behavior in EA and BA than in VA.

\footnotetext{
${ }^{9}$ Some information could be extracted from the prices. For example since the VA price is the second highest bid, one could infer that at least one bidder must have bid this price. However this information is rather vague and moreover it does not give much information about the bidding strategy conditional on the valuation.
} 
Table 5.2: Learning across different treatments.

\begin{tabular}{lccc}
\hline & VA & EA & BA \\
\hline learning not to deviate & & & \\
subjects (WSR, one-sided) & $p=0.02$ & $p=0.02$ & $p=0.02$ \\
sessions (WSR, one-sided) & $p=0.0625$ & $p=0.0625$ & $p=0.0625$ \\
\hline learning not to severely deviate & & & \\
subjects (WSR, one-sided) & $p=0.0013$ & n.s. & $p=0.0013$ \\
sessions (WSR, one-sided) & $p=0.0313$ & n.s. & $p=0.0313$ \\
\hline \# rational bidders in periods 1-3 and 8-10 & 5 & 12 & 15 \\
\# non-learners in periods 1-3 and 8-10 & 17 & 5 & 4 \\
\# non-learners of severe deviations & 7 & 4 & 1 \\
\hline
\end{tabular}

In order to confirm this statistically we calculated the average number of rational subjects (who placed value bids from the beginning) for each session. We can reject the hypothesis of equal number of rational bidders in favor of the hypothesis that there are more rational bidders in EA and BA than in VA (Mann-Whitney-U test, $N=10$ for each comparison, $p \leq 0.03$, one-sided). A comparison between EA and $\mathrm{BA}$ does not result in significant differences.

The second group of subjects did not behave rationally and did also not learn to do so. The numbers of subjects are 17, 5, and 4, for VA, EA, and BA, respectively. Apparently the number of non-learners is much higher in VA than in the other auctions. In the same manner as above we can reject the hypothesis that the number of non-learners is equal between VA and each of the other auctions in favor of the hypothesis that the number of non-learners is higher in VA (Mann-Whitney-U test, $N=10$ for each comparison, $p<0.01$, one-sided).

Table 5.2 gives an overview over the results. The whole analysis of this section was also done with respect to severe deviations. We considered a deviation as severe if the bid / drop-out price / threshold value had a difference of more than 5 to the dominant value. ${ }^{10}$ The learning results were slightly stronger for severe deviations in VA and BA. For EA no significance was found due to the scarcity of severe deviations.

In context of learning it is interesting to mention the observation made for the

\footnotetext{
${ }^{10}$ The precise definition of severe deviation for BA treatment would be: If in at least one round of a period an ask price is more than 5 above the bidder's private valuation and he submits yes then the bidder is considered to severely overbid. If in at least on round of a period an ask price is more than 5 below the bidder's private valuation and he submits no then the bidder is considered to severely underbid.
} 
Vickrey auction. We observed that in VA out of 67 cases where a bidder overbid and won ${ }^{11}$, only $15(22 \%)$ led to a loss for the bidder. Only in 3 out of the 15 cases a bidder revised his behavior after suffering a loss. Moreover, there were 3 bidders who had losses more than once. This illustrates quite well that even from earlier encountered losses bidders hardly learned that overbidding in the Vickrey auction is dominated. This observation goes along with a result of Kagel [31] who found that the feedback mechanism to learn and correct overbidding is weak in the Vickrey auction. To compare it with BA: out of 29 cases where the winner overbid (which is half as much as in VA) the winner lost money in 13 cases (45\%). After suffering from losses only in 4 cases subjects continued overbidding.

Summarizing the findings we get

Result 2. In all treatments subjects learn to adjust their behavior in the direction of the dominant strategy. However, in EA and BA there are significantly more subjects bidding rational right from the beginning and significantly less subjects who do not adjust their behavior.

\subsubsection{Revenue}

According to the theoretical prediction the equilibrium price of an auction, and consequently revenue for the auctioneer must be identical across treatments. Moreover the revenue should be equal to the second highest valuation among the bidders of an auction. To compare the revenues we take a look at the following measurements: first, we are interested in the average resulting revenue; second, the percentage of auctions with the resulting revenue at the predicted level, ${ }^{12}$ and third, the average absolute difference between the resulting and the predicted revenue. Table 5.3 presents the results aggregated over all sessions and periods. We see that the English auction performs "best" in all three measures. With the exception of average revenue the bisection auction comes second while the Vickrey auction comes last.

Testing the results of average revenue on a session level yields no significance. There is, however, a difference in the percentage of auctions with the predicted revenue level between VA and EA as well as between VA and BA. In both cases the null hypothesis of equal percentages is rejected in favor of the alternative hypothesis that the percentage is smaller in VA (Mann-Whitney-U test, $N=10$ for each comparison, $p<0.05$, one-sided). However, we are unable to reject, at conventional significance level, the hypothesis of equality of the percentages for EA and BA. We

\footnotetext{
${ }^{11}$ Almost every second winner overbid his valuation.

${ }^{12}$ Similar as with the definition of a value bid, we accept a deviation by 1 ECU from the predicted revenue.
} 
Table 5.3: Revenue across different treatments.

\begin{tabular}{lccc}
\hline & VA & EA & BA \\
\hline average revenue in ECU & 64.14 & 64.42 & 63.88 \\
\% of auction with pred. rev. level & $42 \%$ & $60 \%$ & $52 \%$ \\
avg. abs. diff. between result and pred. in ECU & 5.58 & 2.82 & 4.39 \\
\hline
\end{tabular}

did not find any significant difference when comparing the percentages of auctions with a revenue higher than the predicted level. Neither did we find any significant difference for the percentages of auctions with a revenue smaller than the predicted level.

Further, the tests show that the average absolute difference between the resulting and the predicted revenue is not the same for EA and BA as well as for EA and VA. In both cases the null hypothesis is rejected in favor of the alternative hypothesis which states that this difference is smaller in EA (Mann-Whitney- $\mathrm{U}$ test, $N=10$ for each comparison, $p<0.05$, one-sided). A comparison of VA and BA does not result in significant differences.

Moreover, for each treatment we investigated how performance in terms of the used revenue measurements changed over time. For this purpose for each measurement we calculated average performance in the beginning of a session (periods 1-3) and at the end of a session (periods 8-10). Testing on the session level yields for EA a significant increase in the percentage of auctions with revenue at the predicted level; for BA a significant decrease in the average absolute difference between the resulting and the predicted revenue (in both cases: Wilcoxon signed-rank test, $N=5, p=0.031)$. For the other measurements and treatments we didn't find any significant changes.

Summarizing this section we achieve:

Result 3. Prices and therefore revenues are significantly more accurate in the English auction than in the other two auctions (according to two measurements, compared to the Vickrey auction, and one measurement, compared to the bisection auction). The accuracy of revenue of the Vickrey and the bisection auctions differs significantly with respect to one measurement. The average revenues of the auctions do not significantly differ from each other. 
Table 5.4: Efficiency across different treatments.

\begin{tabular}{lccc}
\hline & VA & EA & BA \\
\hline allocative efficiency & $86 \%$ & $92 \%$ & $86 \%$ \\
absolute efficiency loss in ECU & 1.22 & 0.63 & 1.59 \\
relative efficiency loss & $1.59 \%$ & $0.83 \%$ & $2.03 \%$ \\
\hline
\end{tabular}

\subsubsection{Efficiency}

An efficient allocation requires assigning an object to the bidder with the highest valuation, because independent of the price this maximizes total social welfare. We compare the auction formats with respect to three different efficiency measures: allocative efficiency, absolute loss of efficiency and relative loss of efficiency. The term allocative efficiency refers simply to the percentage of efficiently allocated objects (i.e. the bidder with the highest valuation has to receive the object). Measuring efficiency in this way does not however reflect the actual magnitude of efficiency lost due to misallocations. If the "wrong" bidder obtains an object, his valuation may be substantially or only slightly below the highest bidder's valuation, causing either dramatic or small welfare losses. Our second and third measures take this into account. With absolute loss of efficiency we refer to the difference between maximum possible welfare and achieved welfare, i.e the difference between the bidders' highest valuation and the valuation of the bidder who wins the auction. The relative loss of efficiency measures the loss of efficiency relative to the maximum possible welfare.

For each measure described above we calculated aggregate results over all sessions and periods (see Table 5.4). Again treatment EA shows the best figures. It is followed by VA and then by BA. ${ }^{13}$ However, there is only one result which is significant: the hypothesis that EA and BA yield the same relative efficiency loss can be rejected in favor of the hypothesis that EA yields a lower relative efficiency loss (Mann-Whitney-U test, $N=10, p=0.047$, one-sided).

Investigating change of efficiency over time on session level we observed significant decreasing of absolute and relative efficiency loss in BA (Wilcoxon signed-rank test, $N=5, p=0.062$ ). For the other measurements and treatments we didn't find any significant changes.

To sum up:

\footnotetext{
${ }^{13}$ While it seems that VA outperforms BA, the results are in favor of BA if we only take the last 3 periods into account: the figures for VA are $82.2 \%, 0.95,1.31 \%$; for BA they are $88.9 \%, 0.77$, $1.02 \%$. None of the measures differs significantly, however.
} 
Result 4. The allocative efficiency of the auctions do not significantly differ from each other. The bisection auction performs worse than the English auction with respect to relative loss of efficiency.

\subsubsection{Post-experimental questionnaire}

Since theory does not predict any significant differences in behavior but differences can be found it makes sense to simply ask subjects about their strategies and thoughts. This was done in a post-experimental questionnaire. ${ }^{14}$ Two problems occur with such kind of data. First the answers were not paid and hence subjects could write anything they want. Second, most questions asked for free text answers and hence it is difficult to provide quantitative summaries. Nevertheless we make the attempt to report a selection of the data. Both authors have investigated the questionnaires independently, classifying the answers into different types. Then we merged our classifications and the corresponding data to the following summary. ${ }^{15}$

Vickrey auction. Twenty two out of 45 subjects indicated that they have chosen (not always from the beginning of the session) the private valuation as a bid. Seven subjects wrote that they have explicitly decided to make bids below their private valuation. Sixteen subjects indicated overbidding as a strategy they have chosen to follow. Half of those 16 bid above their private value only if this value was very high. From the answers of the overbidders we could see that many of them (namely 13) disconnected the probability of getting the object from the price paid. By bidding higher than the private value they thought to increase the probability without influencing the price they had to pay in case they win. They failed to see that it were only the bad cases they won additionally.

English auction. The majority of the subjects, namely 34 out of 45 , indicated that they dropped out of the auction as soon as the price reached (or just past) their valuation. Underbidding and overbidding were mentioned as a chosen strategy by 3 and 7 subjects, respectively. The underbidders attempted to collude. When having a low private value they explicitly dropped out quickly after the period began in order to decrease the price and therefore to increase the profit of the winner. Contrary, 3 of the 7 overbidders exhibited competitive behavior. They stayed active longer in order to increase the price and thus to decrease the profit of the winner, hoping not to become the winner themselves.

Bisection auction. The most frequently mentioned strategies in BA were: submit "No" if the ask price is larger than the private value (21 subjects); and submit

\footnotetext{
${ }^{14}$ The questionnaire can be found in the appendix 5.6.

${ }^{15}$ The raw data is available from the authors on request.
} 
Table 5.5: Questionnaire: subjects' knowledge of auction theory.

\begin{tabular}{lccc}
\hline auction theory & VA & EA & BA \\
\hline knowledge & & & \\
thorough & $10 \%$ & $10 \%$ & $7 \%$ \\
superficial & $26 \%$ & $27 \%$ & $21 \%$ \\
no & $64 \%$ & $63 \%$ & $72 \%$ \\
\hline
\end{tabular}

"Yes" if the current price is smaller than the private value (25 subjects). Eight subjects mentioned that they chose to underbid while 9 mentioned overbidding. Seven subjects mentioned that sometimes, regardless of the private value, they submitted "No" in order to make the price to go down, hoping to increase thereby their potential profit. Four subjects indicated that regardless of their valuation they submitted "Yes" in the first round in order not to be dropped out from the auction immediately. Also with the two last types we see that 13 subjects disconnected the price from the probability to win. They either tried to increase the probability without taking into account the price. Or they tried to lower the price without realizing (or at least underestimating) the risk to be dropped out.

Over all treatments several subjects wrote that the more money they obtained the more risk they were willing to take. Many also explicitly mentioned the avoidance of losses as a primary goal. This was mentioned by 11, 8 and 5 subjects in VA, $\mathrm{BA}$ and EA, respectively.

One of our goals was explicitly to test the auction mechanisms among nonprofessional bidders. However an explicit restriction of the recruitment to subjects without auction knowledge had probably attracted even more experienced subjects, since they might have thought to make a lot of money easily. Consequently we asked subjects about their pre-knowledge ex-post. The answers are summarized in Tables 5.5 and 5.6. ${ }^{16}$ By and large the pre-knowledge seems to be rather similar across treatments with the exception of the experience of online auctions in EA. However, most important for our purpose is that subjects in treatment BA did not have more pre-knowledge then in the other auction formats.

Finally we asked subjects what they consider to be the optimal strategy. Subjects were given a predetermined set of answers which differ only in their degree of deviation from the dominant strategy. Depending on the treatment the answer implied a bid (VA), a drop-out price (EA), or threshold value (BA). The potential

\footnotetext{
${ }^{16}$ The questions about theoretical knowledge and practical experience were only asked in sessions 3-5 of the treatments, so the table contains data on percentage of responded subjects.
} 
Table 5.6: Questionnaire: practical experience of the subjects.

\begin{tabular}{lccc}
\hline online auctions & VA & EA & BA \\
\hline experience & & & \\
frequently & $0 \%$ & $22 \%$ & $0 \%$ \\
sometimes & $30 \%$ & $41 \%$ & $18 \%$ \\
never & $70 \%$ & $37 \%$ & $82 \%$ \\
\hline
\end{tabular}

\begin{tabular}{lccc}
\hline other auctions & VA & EA & BA \\
\hline experience & & & \\
frequently & $0 \%$ & $0 \%$ & $0 \%$ \\
sometimes & $7 \%$ & $8 \%$ & $18 \%$ \\
never & $93 \%$ & $92 \%$ & $82 \%$ \\
\hline
\end{tabular}

Table 5.7: Questionnaire: choice of the optimal strategy.

\begin{tabular}{lccc}
\hline & VA & EA & BA \\
\hline a number that is & & & \\
5 or more below the private value & 2 & 0 & 18 \\
less than 5 below the private value & 4 & 6 & 9 \\
exactly equal to the private value & 25 & 27 & 10 \\
less than 5 above the private value & 8 & 10 & 4 \\
5 or more above the private value & 6 & 2 & 4 \\
\hline
\end{tabular}

answers we gave to the subjects and the data are shown in Table 5.7. ${ }^{17}$

It can be seen that EA subjects produced the most correct answers, closely followed by the VA subjects. The BA subjects gave the correct answer only in $22 \%$ of the cases and therefore differ significantly from the other two treatments (Fisher exact test, $N=90$ for both comparisons, $p<0.001$, two-sided). ${ }^{18}$

It is interesting to compare the answers of the questionnaire with the decisions actually made in the auctions. We say that a subject behaved consistently in a period if her decision in this period was in the same category as the answer. Taking a look at the last three periods we found that in VA 22 subjects (49\%) were consistent in their decisions with what they considered to be optimal. Regarding the last two periods both decisions were in the same category as the answer for 24 subjects $(53 \%)$. Investigating the very last period we see 30 subjects $(67 \%)$ to be consistent. The results in EA turned out to be almost the same, namely 21 (47\%), 22 (49\%) and $30(67 \%)$, respectively.

It is quite difficult to check consistency of subjects in BA, because, due to limited information revelation inherent in the bisection auction, it is rarely possible to find

\footnotetext{
${ }^{17}$ The question for the English auction was framed in such a way that also "exactly equal to the private value" was the right answer.

${ }^{18}$ We neglect any dependence of persons here.
} 
a unique threshold that corresponds to the used strategy. Only for a player who is active during all 7 rounds of an auction the threshold can be found precisely, but if a player drops out or becomes the winner earlier then we can only define a set of thresholds that correspond to actions he made. Since thresholds from this set can be in different categories relatively to the player's valuation we cannot say for sure whether he is consistent or not. ${ }^{19}$ However, it is interesting to notice that among 25 subjects who behaved optimally in more than 6 periods only 9 have answered the question correctly. Fifteen subjects decided that the optimal threshold is below the valuation and 1 subject above the valuation. Among these 16 subjects 13 indicated that making decisions they often or even always thought in terms of threshold strategy.

Taking together the answers in the post-experimental questionnaire we find the following

Result 5. Despite the fact that the questionnaire was not paid according to performance we have no reason to doubt that subjects answered truthfully. The answers give us the following insights:

1. Pre-knowledge of subjects was quite comparable except in the experience with online auctions. There, however, VA and BA subjects had less experience than $E A$ subjects and therefore our positive findings with respect to VA and BA are conservative.

2. In EA, deviations from the truth-telling strategy were mainly driven by social preferences. Cognitive issues seem to be a minor problem. In VA and BA several persons get trapped by the thoughts that it is possible to regard the probability to win and the expected price as independent items to optimize. They thought they could change one item with holding the other constant. This view can cause overbidding as well as underbidding.

3. In BA it seems to be least clear what the optimal strategy is.

\subsection{Summary and conclusions}

The bisection auction is strategically equivalent to the Vickrey and the English auction. However, previous results from the comparison of the Vickrey and the English auction have shown that we should be cautious when drawing conclusions

\footnotetext{
${ }^{19}$ For instance, in the example from Section 2.2 actions of player B could correspond to a threshold strategy with any threshold from set $[0,7]$. Notice that some thresholds from this set are smaller than the valuation of player B and some are larger.
} 
for the behavioral equivalence. Given that the bisection auction does offer some advantages over the others in terms of speed and information revelation, the question rises whether it could be used in practical settings as well. An ultimate answer to this can of course only be given after a real practical application. The experiment can be seen as a first step into this direction.

In the paper we compared the bisection mechanism to two other mechanisms, the Vickrey auction and the English auction. By and large the bisection auction performs somewhere in-between the others. It is significantly better than the Vickrey auction (i.e. closer to theoretical predictions) with respect to the frequency of value-bidding and the accuracy of the price. Compared to the English auction it performs worse with respect to severe deviations from value bidding, the relative loss in efficiency and the absolute difference between resulting and predicted revenue (see Table 5.8 for the full summary on treatments comparison).

Moreover, we investigated how the performance of the auctions changed over time. For the bisection auction we noticed a significant improvement in terms of the frequency of value-bidding, the loss of efficiency and the accuracy of the price (see Table 5.9 for the full summary on change of measurements over time).

A major reason for subjects to deviate from value bidding seems to be the thought that probability to win can be seen independently from the expected price. Subjects who overbid in the Vickrey auction, for example, increase the probability to win but they fail to see that the additional cases are those that give a negative payoff. Some of the subjects see this negative dependence but they underestimate the consequences. ${ }^{20}$ Similar thoughts are possible for the bisection auction: As laid out in Section 5.3.5, some subjects submitted always "Yes" or "No" in the first round in order to increase the probability to win or to decrease the expected price, respectively.

Given that the bisection auction faces the same cognitive trap as the Vickrey auction it is interesting to see that fewer people run into it. We speculate that the reason therefore is that the bisection auction is much more complex than the Vickrey auction. This has been evidenced by the huge number of wrong answers in the questionnaire. People bidding their value know that they will not lose. The more complex design of BA makes them more careful with respect to deviations. This is different in VA where some very simple rules give subjects the impression to overview the situation.

\footnotetext{
${ }^{20}$ For a rational person no estimate is necessary. Overbidding is dominated. People who are, however, not able to analytically understand the Vickrey auction might well just estimate an influence on the expected price.
} 
There is one issue about the practical application that is still open and subject to further research. In the Vickrey and the English auction the price and the winner are determined at the same time. In the bisection auction normally the winner is determined first, then the auction goes on with the determination of the price. Moreover the winner can conclude from the price movements that she has been determined the winner (i.e. she submitted "yes" and nevertheless the ask price drops). In theory as well as in the laboratory there is no communication between the winner and the other bidders. Hence the problem does not exist there, since no bidder except the winner does know that the object is already gone. However, in practical settings the winner might communicate his win and other bidders might drop out of the auction. This could lead to an interesting instance of cartel behavior. On the other hand the remaining bidders might engage in the spiteful action to drive up the price in order to harm the winner. This behavior would have the effect that the bisection auction remains efficient and gives a higher revenue to the auctioneer. Since we have seen some instances of competitive behavior in the EA treatment we may also expect it in real life settings. Eventually this issue is highly speculative and depends on the precise frame the auction takes place in. If the group of bidders knows each other, then the cartel appears to us as the natural consequence. However in competitive market settings where players get for example a long term advantage from driving out competitors from the market, they might try to harm others as severely as possible.

All in all it seems to us that it is worthwhile trying to implement the bisection auction in a real life setting. 
Table 5.8: Comparison of treatments.

BA vs. VA BA vs. EA EA vs. VA

Bidding behavoir

frequency of value-bidding all observations $\mathrm{BA} \succ_{0.01} \mathrm{VA} \quad \mathrm{BA} \approx \mathrm{EA} \quad \mathrm{EA} \succ_{0.01} \mathrm{VA}$ fully informative observations

$\mathrm{BA} \succ_{0.01} \mathrm{VA} \quad \mathrm{BA} \approx \mathrm{EA} \quad \mathrm{EA} \succ_{0.02} \mathrm{VA}$

number of rational bidders

in periods 1-3 and 8-10

$\begin{array}{llll}\text { all deviations } & \mathrm{BA} \succ_{0.05} \mathrm{VA} & \mathrm{BA} \approx \mathrm{EA} & \mathrm{EA} \succ_{0.05} \mathrm{VA} \\ \text { severe deviations } & \mathrm{BA} \succ_{0.05} \mathrm{VA} & \mathrm{EA} \succ_{0.05} \mathrm{BA} & \mathrm{EA} \succ_{0.01} \mathrm{VA}\end{array}$

number of non-learners

in periods $1-3$ and 8-10
all deviations
$\mathrm{BA} \succ_{0.01} \mathrm{VA} \quad \mathrm{BA} \approx \mathrm{EA}$
$\mathrm{EA} \succ_{0.01} \mathrm{VA}$
severe deviations
$\mathrm{BA} \approx \mathrm{VA}$
$\mathrm{EA} \approx \mathrm{BA}$
$\mathrm{EA} \approx \mathrm{VA}$

\section{Efficiency}

$\%$ of auctions with

efficient allocation

$$
\mathrm{BA} \approx \mathrm{VA} \quad \mathrm{BA} \approx \mathrm{EA} \quad \mathrm{EA} \approx \mathrm{VA}
$$

absolute loss of efficiency

$\mathrm{BA} \approx \mathrm{VA}$

$\mathrm{BA} \approx \mathrm{EA}$

$\mathrm{EA} \approx \mathrm{VA}$

relative loss of efficiency

$\mathrm{BA} \approx \mathrm{VA}$

$\mathrm{EA} \succ_{0.05} \mathrm{BA}$

$\mathrm{EA} \approx \mathrm{VA}$

\section{Revenue}

revenue

$\mathrm{BA} \approx \mathrm{VA}$

$\mathrm{BA} \approx \mathrm{EA}$

$\mathrm{EA} \approx \mathrm{VA}$

$\%$ of auctions with revenue at the predicted level

$\mathrm{BA} \succ_{0.05} \mathrm{VA}$

$\mathrm{BA} \approx \mathrm{EA}$

$\mathrm{EA} \succ_{0.05} \mathrm{VA}$

absolute difference between resulting and predicted revenue

$\mathrm{BA} \approx \mathrm{VA}$

$\mathrm{EA} \succ_{0.05} \mathrm{BA}$

$\mathrm{EA} \succ_{0.05} \mathrm{VA}$

Note: $\mathrm{BA} \succ_{0.05} \mathrm{VA}$ means that the bisection auction outperforms the Vickrey auction (in terms of a particular measurement) at the level of significance $0.05 . \mathrm{EA} \approx \mathrm{BA}$ means that there is no significant (any level less than 0.1) difference between the performance (in terms of a particular measurement) of the English and the bisection auctions. 
Table 5.9: Comparison of measurements of a particular treatment over time.

\begin{tabular}{llll}
\hline BA & EA & VA \\
\hline
\end{tabular}

\section{Bidding behavior}

$\begin{array}{llll}\text { underbidding } & \mathrm{BA}_{e} \succ_{0.05} \mathrm{BA}_{b} & \mathrm{EA}_{e} \succ_{0.05} \mathrm{EA}_{b} & \mathrm{VA}_{e} \succ_{0.07} \mathrm{VA}_{b} \\ \text { value bidding } & \mathrm{BA}_{e} \succ_{0.07} \mathrm{BA}_{b} & \mathrm{EA}_{e} \succ_{0.07} \mathrm{EA}_{b} & \mathrm{VA}_{e} \succ_{0.07} \mathrm{VA}_{b} \\ & \mathrm{BA}_{b} \approx \mathrm{BA}_{e} & \mathrm{EA}_{b} \approx \mathrm{EA}_{e} & \text { n.e.d. }\end{array}$

\section{Efficiency}

$\%$ of auctions with

efficient allocation

n.e.d

$\mathrm{EA}_{b} \approx \mathrm{EA}_{e} \quad \mathrm{VA}_{b} \approx \mathrm{VA}_{e}$

absolute loss of efficiency

$\mathrm{BA}_{e} \succ_{0.07} \mathrm{BA}_{b}$

$\mathrm{EA}_{b} \approx \mathrm{EA}_{e}$

$\mathrm{VA}_{b} \approx \mathrm{VA}_{e}$

relative loss of efficiency

$\mathrm{BA}_{e} \succ_{0.07} \mathrm{BA}_{b}$

$\mathrm{EA}_{b} \approx \mathrm{EA}_{e} \quad \mathrm{VA}_{b} \approx \mathrm{VA}_{e}$

\section{Revenue}

$\%$ of auctions with revenue

at the predicted level

$\mathrm{BA}_{b} \approx \mathrm{BA}_{e} \quad \mathrm{EA}_{e} \succ_{0.05} \mathrm{EA}_{b} \quad \mathrm{VA}_{b} \approx \mathrm{VA}_{e}$

absolute difference between

resulting and predicted revenue

$\mathrm{BA}_{e} \succ_{0.05} \mathrm{BA}_{b}$

$\mathrm{EA}_{b} \approx \mathrm{EA}_{e}$

$\mathrm{VA}_{b} \approx \mathrm{VA}_{e}$

\section{Learning process}

all deviations

severe deviations
$\mathrm{BA}_{e} \succ_{0.05} \mathrm{BA}_{b}$

$\mathrm{BA}_{e} \succ_{0.01} \mathrm{BA}_{b}$
$\mathrm{EA}_{e} \succ_{0.05} \mathrm{EA}_{b}$

$\mathrm{EA}_{b} \approx \mathrm{EA}_{e}$
$\mathrm{VA}_{e} \succ_{0.05} \mathrm{VA}_{b}$

$\mathrm{VA}_{e} \succ_{0.01} \mathrm{VA}_{b}$

Note: $\mathrm{BA}_{e} \succ_{0.05} \mathrm{BA}_{b}$ means that the performance of the bisection auction (in terms of a particular measurement) is improved (at the level of significance 0.05) at the end in comparison with the beginning. $\mathrm{EA}_{b} \approx \mathrm{EA}_{e}$ means that there is no significant (any level less than 0.1) difference between the performance of the English auction (in terms of a particular measurement) in the beginning and at the end. N.e.d. means that there is not enough informative data to make any conclusion. 


\subsection{Appendix 1: Subjects instructions}

Dear participant, thank you for taking part in this experiment! It will last about 1 hour. You will be compensated according to your performance. In order to ensure that the experiment takes place in an optimal setting, we would like to ask you to follow the general rules during the whole experiment:

- Do not communicate with your fellow students!

- Please switch off your mobile phone!

- Please read the instructions carefully. It is important that you understand the rules of the experiment. If something is not explained well, please raise your hand. We will then answer your questions privately. The instructions are identical for all participants.

- You may make notes on this instruction sheet if you wish.

- After the experiment please remain seated till you are paid off.

- If you do not obey the rules, the data becomes useless for us. Therefore we will have to exclude you from this experiment and you will not receive any compensation.

Your decisions are anonymous. Neither your fellow students nor anybody else will ever learn them from us.

The experiment consists of 10 periods. In each period the participants are randomly matched into groups of three bidders. In each group a fictitious commodity is auctioned off. Before the auction starts you will receive information about your private value for the commodity, expressed in Experimental Currency Unit (ECU). The private values are independently and randomly determined. Thus, they may be different for different bidders. Each integer value between 32 and 95 is equally likely. If you win the auction you do not receive the commodity but you receive an amount of money equal to your private value. In return you have to pay the price resulting from the auction.

Who wins the auction and what price the winner has to pay is determined in the following way:

This part of the instructions is different for each treatment. See below the corresponding parts.

ECUs are transformed into Euros according to the following conversion rate: 1 Euro $=4.5$ ECU. You will obtain an initial endowment of 3 Euro. If you 
make losses in an auction these will be deducted from your previous gains (or from your initial endowment). You will receive your final profit in cash at the end of the experiment.

Before the experiment starts please answer the following questions:

1. Assume three bidders Ann, Bert and Chris take part in the experiment. Ann gets informed about her private value which is 62 . What does Ann know about the private values of Bert and Chris?

(a) Bert and Chris have exactly the same private values, i.e. 62 .

(b) Bert and Chris have different private values, i.e. their values are between 32 and 95 but not 62 .

(c) Bert and Chris may have the same but may also have different values, i.e their values are between 32 and 95 including 62 .

2. Assume that the participants have the following private values: Ann 57, Bert 41 and Chris 75. Assume further that Ann wins the auction and that the price is 48 .

What are the profits of the three participants?

Ann:

Bert:

Chris:

3. Assume that the participants have the following private values: Ann 39, Bert 77 and Chris 77. Assume further that Bert wins the auction and that the price is 83 .

What are the profits of the three participants?

Ann:

Bert:

Chris:

After the experiment, we would like to ask you to complete a short questionnaire. Thank you again and good luck with the experiment! 


\section{Part of the instructions corresponding to the Vickrey auction.}

After you observe your private value, you have to place a bid which is allowed to be between 0 and 127 ECU. After every bidder in your group has placed his bid the winner and the price at which he obtains the commodity is determined. The bidder with the highest bid is the winner. The price he has to pay is equal to the second highest bid. If more than 1 bidder submitted the same highest bid, the winner is determined randomly among these bidders. The price he has to pay is, again, equal to the second highest bid which in this case coincides with the highest bid.

The profit of the winner is determined as the difference between his private value and the price. If his private value is larger than the price, he receives this difference. If his value is less than the price, he has to pay this difference. The other bidders do not receive anything and do not pay anything.

Please make your decisions carefully - your reward will depend on your performance during the experiment. Note that you can make losses, but it is always possible, however, to bid in such a way that you avoid losses for sure.

You will get information and make your decisions via the computer terminal. In each period you go through the following rounds:

- You observe your private value for the commodity. You will not be informed about the values of the others, just as they do not know your private value.

- You submit your bid which is allowed to be between 0 and 127 ECU.

- You observe whether or not you bought the commodity, the price, your gains /losses in this auction and in total including all previous auctions.

- A new period starts. You will be randomly matched to two other participants. 


\section{Part of the instructions corresponding to the English auction.}

The auction starts with a price of 0 . Every 2 seconds the current price is increased by 1 . As long as you are not reacting we assume that you are active, that means you are bidding the current price. If the price reaches a level which you are not willing to bid anymore you have to click the button "DROP OUT". From this moment on you are not active anymore. For active bidders the price continues to increase. The auction stops as soon as there is only one active bidder left. The last remaining bidder becomes the winner. The price the winner has to pay is 1 ECU below the price where the second last bidder dropped out. If several bidders drop out simultaneously and no bidder is left active, the winner is determined randomly among these bidders and the price is 1 ECU below the price where they dropped out. If upon reaching the maximal price of $128 \mathrm{ECU}$ there is more than one active bidder, then the commodity is randomly allocated among these active bidders for a price of $127 \mathrm{ECU}$.

The profit of the winner is determined as the difference between his private value and the price. If his private value is larger than the price, he receives this difference. If his value is less than the price, he has to pay this difference. The other bidders do not receive anything and do not pay anything.

Please make your decisions carefully - your reward will depend on your performance during the experiment. Note that you can make losses, but it is always possible, however, to bid in such a way that you avoid losses for sure.

You will get information and make your decisions via the computer terminal. In each period you go through the following rounds:

- You observe your private value for the commodity. You will not be informed about the values of the others, just as they do not know your private value.

- You observe successive change of the price and indicate your dropping out by clicking the button "DROP OUT" at the price you are not willing to bid any more.

- After the period ends you observe whether or not you bought the commodity, the price, your gains/losses in this period and in total including all previous periods.

- A new period starts. You will be randomly matched to two other participants. 


\section{Part of the instructions corresponding to the bisection auction.}

One period consists of 7 rounds. For each round the interval where the bidders are competing for the commodity is determined. The ask price in a round is set to the middle of this interval. In the first round the interval is $[0,128)$ and the ask price is equal to 64 . All bidders receive the status "Active". Active bidders are asked whether they are willing to bid the current ask price for the commodity. A bidder replies "YES" to announce his willingness and " $N O$ " to announce his unwillingness. After all bidders submit their decision, the interval and the ask price of the next round as well as the status of bidders are determined according to the following rule:

- In case 3 "yes"-bids are submitted: All three bidders are willing to bid the ask price for one available commodity. It means that at the ask price demand exceeds supply and therefore we can concentrate our search to the upper half of the previous interval. The price goes up to the middle of this new interval. It means that in round 2 the interval will be $[64,128)$ and the price 96 . All active bidders remain active.

- In case 2 "yes"-bids and 1 "no"-bid are submitted: Two bidders are willing to bid the ask price for one available commodity. It means that at the ask price demand exceeds supply and therefore, again, we can concentrate our search to the upper half of the previous interval. The price goes up to the middle of this new interval. It means that in round 2 the interval will be $[64,128)$ and the price 96 . The bidders that are active in the next round are the ones that submitted "yes"-bids. The bidder with a "no"-bid drops out of the auction. His status remains "Dropped out" till the end of the period. He is not free anymore to choose between "yes" and "no". Since the prices in all next rounds will be larger than the price that this bidder declined when he became a drop out, his decisions in all forthcoming rounds are considered to be "no".

- In case 1 "yes"-bid and 2 "no"-bids are submitted: There is only one bidder who is willing to bid the ask price. The bidder with a "yes"-bid becomes the winner of the auction. His status remains "The winner" till the end of the period. The bidders that are active in the next round are the ones that submitted "no"-bids. By their future bids the price the winner has to pay is determined. The new interval is set equal to the lower half of the previous interval. So the price goes down to the middle of this new interval. It means that in round 2 the interval will be $[0,64)$ and the price 32 . The winner is not 
free to choose between "yes" and "no". Since the prices in all next rounds will be lower than the price that this bidder agreed on when he became the winner, his decisions in all forthcoming rounds are considered to be "yes".

- In case 3 "no"-bids are submitted: No bidder is willing to bid the ask price for the commodity. It means that at the ask price supply exceeds demand and therefore we can concentrate our search to the lower half of the previous interval. So the price, again, goes down to the middle of this new interval. It means that in round 2 the interval will be $[0,64)$ and the price 32 . All active bidders remain active.

The way to decide about the status of bidders, the change of interval and the price is the same in all rounds. Depending on submitted bids we subsequently restrict attention to either the lower half or to the upper half of the previous interval. The only information bidders get after each round is the new interval, the new price and own status. The bidders are not informed about the status of others, so during the period you do not get to know whether there are drop outs or the winner among the other bidders. Thus, an active bidder does not know whether he is competing for the commodity or he is determining the winner's price. After round 7 the new interval is determined. The price the winner has to pay is equal to the lower bound of this interval. If the winner was not found during 7 rounds (i.e. if in no round exactly one bidder submitted a "yes"-bid), the status of the bidders after the last round is determined and the commodity is randomly allocated to one of the remaining active bidders.

The profit of the winner is determined as the difference between his private value and the price. If his private value is larger than the price, he receives this difference. If his value is less than the price, he has to pay this difference. The other bidders do not receive anything and do not pay anything.

Please make your decisions carefully - your reward will depend on your performance during the experiment. Note that you can make losses, but it is always possible, however, to bid in such a way that you avoid losses for sure.

You will get information and make your decisions via the computer terminal. In each period you go through the following phases of the auction:

- You observe your private value for the commodity. You will not be informed about the values of the others, just as they do not know your private value.

- ROUND 1: You are "Active". You observe the initial interval $[0,128)$ and the initial price 64 . You have to indicate your decision "YES, I'm willing to bid 
this price for the commodity" or "NO, I'm not willing to bid this price for the commodity" by clicking the corresponding button.

- ROUNDS 2-7: You observe your current status that can be "Active", "Dropped out" or "The winner". You observe the current interval and the current price. If you are active you have to indicate your decision "YES" or "NO" for the current price. If you are a drop out or the winner you are not allowed to submit bids anymore.

- After round 7 the period ends and you observe whether or not you bought the commodity, the winning price, your gains/losses in this period and in total including all previous periods.

- A new period starts. You will be randomly matched to two other participants. 


\subsection{Appendix 2: Post-experimental questionnaire}

Subject ID (see the card you have drawn in the beginning):

Year of birth:

Gender:

Nationality:

Study:

In the following we are interested to learn how you reached your decision. Please answer the following questions as precisely as possible.

What factors did influence your decision? What information did you use?

Given the information and factors mentioned above, how did you make up your strategy?

Did you encounter problems in particular with the software? If yes, what problems?

Did you ever deal with auctions in one of your courses?

$\circ$ yes, thoroughly

○ yes, but superficially

○ never

Do or did you participate in online auctions (e.g. www.ebay.com)?

$\circ$ frequently

- sometimes

○ never

Do or did you participate in other but online auctions?

$\circ$ frequently

○ sometimes

$\circ$ never

This last part of the questionnaire is different for each treatment. See below the corresponding parts. 


\section{Part of the questionnaire corresponding to BA treatment.}

Consider the following strategy: Given your private value of the commodity you chose a number. Whenever the ask price is equal to or below this number you submit "Yes". If the ask price is above this number you submit "No".

Did you use such a threshold strategy?

- always

$\circ$ often

○ sometimes

○ never

Suppose you cannot participate in the auction yourself but you can submit a number to a software agent who bids for you according to the strategy described in the question above.

Given that you know your private value, which number would maximize your payoff?

- a number that is 5 or more below the private value

- a number that is less than 5 below the private value

- the number exactly equal to the private value

- a number that is less than 5 above the private value

- a number that is 5 or more above the private value

\section{Part of the questionnaire corresponding to VA treatment.}

Which strategy do you consider to maximize your payoff?

- submit a bid that is 5 or more below the private value

- submit a bid that less than 5 below the private value

- submit a bid exactly equal to the private value

- submit a bid that is less than 5 above the private value

- submit a bid that is 5 or more above the private value 


\section{Part of the questionnaire corresponding to EA treatment.}

Which strategy do you consider to maximize your payoff?

- drop out if the price exceeds a value which is 5 or more below the private value

- drop out if the price exceeds a value which is less than 5 below the private value

- drop out if the price exceeds the private value

- drop out if the price exceeds a value which is less than 5 above the private value

- drop out if the price exceeds a value which is 5 or more above the private value 


\subsection{Appendix 3: Tables with data used for the con- ducted tests}

session 1 session $2 \quad$ session $3 \quad$ session 4 session 5

\begin{tabular}{llllll}
\hline \hline & & & & & \\
$\mathrm{BA}$ & $64.4 \%$ & $81.1 \%$ & $68.9 \%$ & $75.5 \%$ & $68.9 \%$ \\
$\mathrm{BA}_{b}$ & $48.1 \%$ & $85.2 \%$ & $74.1 \%$ & $70.4 \%$ & $59.2 \%$ \\
$\mathrm{BA}_{e}$ & $88.9 \%$ & $88.9 \%$ & $74.1 \%$ & $81.5 \%$ & $70.4 \%$ \\
\hline & & & & & \\
$\mathrm{VA}$ & $53.3 \%$ & $26.6 \%$ & $45.5 \%$ & $60.0 \%$ & $54.4 \%$ \\
$\mathrm{VA}_{b}$ & $33.3 \%$ & $25.9 \%$ & $25.9 \%$ & $59.2 \%$ & $51.8 \%$ \\
$\mathrm{VA}_{e}$ & $59.2 \%$ & $25.9 \%$ & $70.4 \%$ & $66.7 \%$ & $55.5 \%$ \\
\hline & & & & & \\
$\mathrm{EA}$ & $69.9 \%$ & $76.7 \%$ & $68.9 \%$ & $76.7 \%$ & $75.5 \%$ \\
$\mathrm{EA}_{b}$ & $62.9 \%$ & $77.8 \%$ & $62.9 \%$ & $70.4 \%$ & $59.2 \%$ \\
$\mathrm{EA}_{e}$ & $62.9 \%$ & $81.5 \%$ & $70.4 \%$ & $88.9 \%$ & $92.6 \%$ \\
\hline
\end{tabular}

Table 5.10: Frequency of value bidding.

session 1 session 2 session 3 session 4 session 5

\begin{tabular}{llllll}
\hline \hline & & & & & \\
BA & $65.7 \%$ & $66.6 \%$ & $63.5 \%$ & $71.9 \%$ & $58.9 \%$ \\
VA & $53.3 \%$ & $26.6 \%$ & $45.5 \%$ & $60.0 \%$ & $54.4 \%$ \\
EA & $71.0 \%$ & $65.6 \%$ & $53.4 \%$ & $67.7 \%$ & $63.3 \%$ \\
\hline
\end{tabular}

Table 5.11: Frequency of value-bidding among the players with fully informative bids. 
session $1 \quad$ session $2 \quad$ session $3 \quad$ session $4 \quad$ session 5

\begin{tabular}{lrrrrr}
\hline \hline & & & & \\
$\mathrm{BA}$ & $21.1 \%$ & $12.2 \%$ & $15.6 \%$ & $3.3 \%$ & $20.0 \%$ \\
$\mathrm{BA}_{b}$ & $33.3 \%$ & $7.4 \%$ & $14.8 \%$ & $3.7 \%$ & $25.9 \%$ \\
$\mathrm{BA}_{e}$ & $11.1 \%$ & $3.7 \%$ & $7.4 \%$ & $0.0 \%$ & $18.5 \%$ \\
\hline & & & & & \\
$\mathrm{VA}$ & $17.8 \%$ & $23.3 \%$ & $41.1 \%$ & $11.1 \%$ & $17.8 \%$ \\
$\mathrm{VA}_{b}$ & $37.0 \%$ & $29.6 \%$ & $66.7 \%$ & $18.5 \%$ & $48.1 \%$ \\
$\mathrm{VA}_{e}$ & $11.1 \%$ & $29.6 \%$ & $22.2 \%$ & $3.7 \%$ & $3.7 \%$ \\
\hline & & & & \\
$\mathrm{EA}$ & $7.8 \%$ & $15.5 \%$ & $18.9 \%$ & $18.9 \%$ & $23.3 \%$ \\
$\mathrm{EA}_{b}$ & $11.1 \%$ & $18.5 \%$ & $29.6 \%$ & $18.5 \%$ & $40.7 \%$ \\
$\mathrm{EA}_{e}$ & $3.7 \%$ & $7.4 \%$ & $3.7 \%$ & $7.4 \%$ & $7.4 \%$ \\
\hline
\end{tabular}

Table 5.12: Frequency of underbidding.

session $1 \quad$ session $2 \quad$ session $3 \quad$ session $4 \quad$ session 5

\begin{tabular}{lrrrrr}
\hline \hline & & & & & \\
$\mathrm{BA}$ & $14.4 \%$ & $6.7 \%$ & $15.5 \%$ & $21.1 \%$ & $11.1 \%$ \\
$\mathrm{BA}_{b}$ & $18.5 \%$ & $7.4 \%$ & $11.1 \%$ & $25.9 \%$ & $14.8 \%$ \\
$\mathrm{BA}_{e}$ & $7.4 \%$ & $7.4 \%$ & $18.5 \%$ & $18.5 \%$ & $11.1 \%$ \\
\hline & & & & & \\
$\mathrm{VA}$ & $28.9 \%$ & $50.0 \%$ & $13.3 \%$ & $28.9 \%$ & $27.7 \%$ \\
$\mathrm{VA}_{b}$ & $29.6 \%$ & $44.4 \%$ & $7.4 \%$ & $22.2 \%$ & $0.0 \%$ \\
$\mathrm{VA}_{e}$ & $29.6 \%$ & $44.4 \%$ & $7.4 \%$ & $29.6 \%$ & $48.1 \%$ \\
\hline & & & & & \\
$\mathrm{EA}$ & $22.2 \%$ & $7.8 \%$ & $12.2 \%$ & $4.4 \%$ & $1.1 \%$ \\
$\mathrm{EA}_{b}$ & $25.9 \%$ & $3.7 \%$ & $7.4 \%$ & $11.1 \%$ & $0.0 \%$ \\
$\mathrm{EA}_{e}$ & $33.4 \%$ & $11.1 \%$ & $25.9 \%$ & $3.7 \%$ & $0.0 \%$ \\
\hline
\end{tabular}

Table 5.13: Frequency of overbidding. 
session $1 \quad$ session $2 \quad$ session 3 session $4 \quad$ session 5

all deviations

$\begin{array}{llllll}\text { BA } & 1 & 5 & 4 & 3 & 2 \\ \text { VA } & 1 & 0 & 0 & 2 & 2 \\ \text { EA } & 2 & 3 & 3 & 1 & 3\end{array}$

severe deviations

\begin{tabular}{llllll} 
BA & 1 & 6 & 5 & 5 & 4 \\
VA & 3 & 0 & 0 & 2 & 3 \\
EA & 6 & 6 & 6 & 6 & 6 \\
\hline
\end{tabular}

Table 5.14: Number of subjects who didn't deviate neither in the beginning of a session nor at the end.

session 1 session $2 \quad$ session 3 session $4 \quad$ session 5

all deviations

$\begin{array}{llllll}\text { BA } & 2 & 0 & 0 & 1 & 1 \\ \text { VA } & 4 & 5 & 3 & 3 & 2 \\ \text { EA } & 1 & 1 & 2 & 1 & 1\end{array}$

severe deviations

\begin{tabular}{llllll} 
BA & 1 & 1 & 1 & 1 & 0 \\
VA & 3 & 3 & 0 & 1 & 0 \\
EA & 0 & 0 & 0 & 0 & 1 \\
\hline
\end{tabular}

Table 5.15: Number of subjects who deviated in the beginning of a session and didn't change behavior at the end. 
session $1 \quad$ session $2 \quad$ session $3 \quad$ session $4 \quad$ session 5

\begin{tabular}{llllll}
\hline \hline & & & & & \\
BA & 62.7 & 62.9 & 64.4 & 66.6 & 62.8 \\
VA & 60.6 & 67.5 & 62.7 & 66.3 & 63.6 \\
EA & 65.3 & 64.7 & 67.6 & 62.2 & 62.3 \\
\hline
\end{tabular}

Table 5.16: Average revenue of auctions.

session $1 \quad$ session $2 \quad$ session $3 \quad$ session $4 \quad$ session 5

\begin{tabular}{llllll}
\hline \hline & & & & & \\
$\mathrm{BA}$ & $53 \%$ & $60 \%$ & $47 \%$ & $53 \%$ & $47 \%$ \\
$\mathrm{BA}_{b}$ & $33 \%$ & $67 \%$ & $55 \%$ & $44 \%$ & $55 \%$ \\
$\mathrm{BA}_{e}$ & $78 \%$ & $78 \%$ & $55 \%$ & $67 \%$ & $55 \%$ \\
\hline & & & & & \\
$\mathrm{VA}$ & $43 \%$ & $27 \%$ & $40 \%$ & $43 \%$ & $57 \%$ \\
$\mathrm{VA}_{b}$ & $33 \%$ & $33 \%$ & $22 \%$ & $33 \%$ & $44 \%$ \\
$\mathrm{VA}_{e}$ & $22 \%$ & $44 \%$ & $55 \%$ & $55 \%$ & $66 \%$ \\
\hline & & & & & \\
$\mathrm{EA}$ & $43 \%$ & $73 \%$ & $63 \%$ & $63 \%$ & $57 \%$ \\
$\mathrm{EA}$ & $44 \%$ & $67 \%$ & $55 \%$ & $44 \%$ & $44 \%$ \\
$\mathrm{EA}_{e}$ & $66 \%$ & $89 \%$ & $67 \%$ & $67 \%$ & $78 \%$ \\
\hline
\end{tabular}

Table 5.17: Percentage of auctions with revenue at the predicted level. 
session 1 session 2 session 3 session 4 session 5

\begin{tabular}{lrrrrr}
\hline \hline & & & & & \\
$\mathrm{BA}$ & 4.77 & 3.51 & 3.87 & 5.63 & 3.43 \\
$\mathrm{BA}_{b}$ & 8.55 & 3.00 & 6.11 & 5.78 & 4.44 \\
$\mathrm{BA}_{e}$ & 1.67 & 2.60 & 5.88 & 1.67 & 2.67 \\
\hline & & & & & \\
$\mathrm{VA}$ & 7.97 & 6.33 & 6.23 & 4.83 & 2.53 \\
$\mathrm{VA}$ & 5.00 & 7.78 & 11.00 & 5.55 & 4.44 \\
$\mathrm{VA}_{e}$ & 17.00 & 5.11 & 1.78 & 3.11 & 1.55 \\
\hline & & & & & \\
$\mathrm{EA}$ & 4.83 & 1.53 & 1.97 & 3.07 & 2.71 \\
$\mathrm{EA}_{b}$ & 2.44 & 2.89 & 2.44 & 2.66 & 4.44 \\
$\mathrm{EA}_{e}$ & 4.89 & 0.89 & 1.53 & 4.89 & 1.22 \\
\hline
\end{tabular}

Table 5.18: Average difference between the resulting and the predicted revenue (in absolute value).

session $1 \quad$ session $2 \quad$ session $3 \quad$ session $4 \quad$ session 5

\begin{tabular}{lrrrrr}
\hline \hline & & & & & \\
$\mathrm{BA}$ & $87.0 \%$ & $97.0 \%$ & $80.0 \%$ & $80.0 \%$ & $87.0 \%$ \\
$\mathrm{BA}_{b}$ & $77.8 \%$ & $100.0 \%$ & $77.8 \%$ & $88.9 \%$ & $88.9 \%$ \\
$\mathrm{BA}_{e}$ & $100.0 \%$ & $100.0 \%$ & $77.8 \%$ & $88.9 \%$ & $88.9 \%$ \\
\hline & & & & & \\
$\mathrm{VA}$ & $90.0 \%$ & $67.0 \%$ & $83.0 \%$ & $87.0 \%$ & $100.0 \%$ \\
$\mathrm{VA}_{b}$ & $88.9 \%$ & $55.5 \%$ & $77.8 \%$ & $88.9 \%$ & $100.0 \%$ \\
$\mathrm{VA}_{e}$ & $77.8 \%$ & $77.8 \%$ & $88.9 \%$ & $66.7 \%$ & $100.0 \%$ \\
\hline & & & & & \\
$\mathrm{EA}$ & $80.0 \%$ & $97.0 \%$ & $97.0 \%$ & $97.0 \%$ & $90.0 \%$ \\
$\mathrm{EA}_{b}$ & $88.9 \%$ & $88.9 \%$ & $88.94 \%$ & $100.0 \%$ & $77.8 \%$ \\
$\mathrm{EA}_{e}$ & $77.8 \%$ & $100.0 \%$ & $100.0 \%$ & $88.9 \%$ & $100.0 \%$ \\
\hline
\end{tabular}

Table 5.19: Percentage of auctions with the efficient allocation. 
session 1 session 2 session 3 session 4 session 5

\begin{tabular}{llllll}
\hline \hline & & & & & \\
$\mathrm{BA}$ & 3.00 & 0.17 & 1.73 & 1.60 & 1.43 \\
$\mathrm{BA}_{b}$ & 5.89 & 0.00 & 3.11 & 2.22 & 2.33 \\
$\mathrm{BA}_{e}$ & 0.00 & 0.00 & 1.55 & 1.44 & 0.89 \\
\hline & & & & & \\
$\mathrm{VA}$ & 0.53 & 2.33 & 1.57 & 1.57 & 0.00 \\
$\mathrm{VA}_{b}$ & 0.33 & 4.11 & 3.44 & 2.22 & 0.00 \\
$\mathrm{VA}_{e}$ & 0.88 & 0.33 & 0.55 & 3.00 & 0.00 \\
\hline & & & & & \\
$\mathrm{EA}$ & 1.23 & 0.20 & 0.43 & 0.20 & 1.07 \\
$\mathrm{EA}_{b}$ & 0.22 & 0.66 & 1.44 & 0.00 & 2.11 \\
\hline
\end{tabular}

Table 5.20: Average loss of efficiency (in absolute value).

session 1 session $2 \quad$ session 3 session 4 session 5

\begin{tabular}{llllll}
\hline \hline & & & & & \\
$\mathrm{BA}$ & $3.62 \%$ & $0.33 \%$ & $2.45 \%$ & $2.08 \%$ & $1.66 \%$ \\
$\mathrm{BA}_{b}$ & $6.55 \%$ & $0.00 \%$ & $5.09 \%$ & $2.81 \%$ & $2.51 \%$ \\
$\mathrm{BA}_{e}$ & $0.00 \%$ & $0.00 \%$ & $1.68 \%$ & $2.26 \%$ & $1.16 \%$ \\
\hline & & & & & \\
$\mathrm{VA}$ & $0.83 \%$ & $3.28 \%$ & $1.89 \%$ & $1.94 \%$ & $0.00 \%$ \\
$\mathrm{VA}_{b}$ & $0.47 \%$ & $5.17 \%$ & $3.93 \%$ & $2.58 \%$ & $0.00 \%$ \\
$\mathrm{VA}_{e}$ & $1.27 \%$ & $0.48 \%$ & $0.89 \%$ & $3.87 \%$ & $0.00 \%$ \\
\hline & & & & & \\
$\mathrm{EA}$ & $1.57 \%$ & $0.22 \%$ & $0.67 \%$ & $0.34 \%$ & $1.34 \%$ \\
$\mathrm{EA}_{b}$ & $0.32 \%$ & $0.74 \%$ & $2.22 \%$ & $0.00 \%$ & $2.92 \%$ \\
$\mathrm{EA}_{e}$ & $1.98 \%$ & $0.00 \%$ & $0.00 \%$ & $1.13 \%$ & $0.00 \%$ \\
\hline
\end{tabular}

Table 5.21: Average relative loss of efficiency. 



\section{Part II}

\section{Bisection auctions in the continuous setting}





\section{Chapter 6}

\section{Inefficiency of equilibria in query auctions with continuous valuations}

In this chapter we investigate the allocative efficiency of query auctions in the context of continuous valuations. We show that in an ex post equilibrium of any individually rational query auction the item will necessarily be assigned inefficiently with positive probability, unless one accepts the unrealistic phenomenon that the auction runs an infinite number of queries for almost all realizations of valuations. It means that in the continuous setting query auctions, being a good tool for reducing preference revelation, does not allow achieving full allocative efficiency. ${ }^{1}$

\subsection{Introduction}

The Vickrey auction and the iterative auctions strategically equivalent to it have been shown to be a satisfactory way to sell a single indivisible item in the setting of integer valuations. It is however sometimes desirable to be able to run an auction without an a-priori agreement on the discretization of bids. For example, in a computerized bidding environment in multi-agent systems the precision with which bidding agents represent their valuations might be unknown, and therefore preferably be left unspecified. Also time constraints - an issue of some importance in auctions like the UMTS auctions (see Moldovanu and Jehiel [43], van Damme [58]) - can thus be captured, since time restrictions might force an auctioneer to determine valuations only up to a level of precision that is not of the same order

\footnotetext{
${ }^{1}$ The results of this chapter were first presented in Grigorieva et al. [23].
} 
of magnitude in which bidders do, or would like to, express their valuations. The possibility of arbitrary fine representation of valuations can be modeled by allowing valuations to take on continuous values.

In this chapter we are concerned with the issue of designing iterative implementation of the Vickrey auction for the setting of continuous valuations. We consider multi-round query auctions where the auctioneer sequentially queries the bidders about specific aspects of their valuations, offering them the opportunity to take one of a finite set of actions as an answer to the query. We prove that, when bidders have continuous valuations, any ex-post equilibrium in an ex-post individually rational query auction that ends with positive probability after a finite number of queries, can not be fully efficient. It means that in the continuous setting the execution of the Vickrey auction by means of multi-round queries is not possible, unless we have infinite running time. In other words, any implementation of the Vickrey auction by means of a query auction in a model with continuous valuations will necessarily have an infinite running time for almost all realizations of valuations, and is hence not a feasible option in any practical sense.

Related literature Iterative models with a finite set of possible bids in the setting of continuous valuations have received some attention in the auction literature. One such a model, namely an English auction with discrete bid levels has been studied extensively. Yamey [61] first considered this auction format and commented that introducing of discrete bid levels appear to have the effect of speeding up the auction proceedings and hence reduce the costs of both the auctioneer and the bidders. However, such restriction of possible bid levels causes many well known results from the continuous bid auction literature to fail. For example, the bidders within the auction no longer have a dominant bidding strategy ${ }^{2}$ (see $\mathrm{Yu}$ [62]) and, as the item is no longer guaranteed to be allocated to the bidders with the highest valuation, the Revenue Equivalence Theorem ${ }^{3}$ no longer applies (see Chwe [7]), and thus the revenue that the auction generates depends on the specific implementation details, such as the number and the distribution of the discrete bid levels. To elaborate on this issue, Rothkopf and Hastard [51] provide a full discussion of how the choice of discrete levels affects the expected revenue of the auction. For a number of very limited simple cases, they address the question of revenue maximizing design of an English auction with discrete bid levels. David et al. [16] extend this work and derive general results that indicate how the discrete bid levels should be set in order

\footnotetext{
${ }^{2}$ Though recently David et al. [16] proposed to modify the standard auction rules in such a way that a dominant strategy equilibrium exists.

${ }^{3}$ This theorem states that all efficient auction formats yield the same expected revenue in equilibrium.
} 
to maximize the revenue of the auctioneer. Kress and Boutilier [33] are the first who addressed revelation properties of ascending price auctions with discrete bid levels.

\subsection{Preliminaries}

Here we briefly discuss some known results on auction design and the notions that we use in this chapter. A single indivisible object is being sold to a set $N=\{1, \ldots, n\}$ of bidders by means of a deterministic auction. The set of actions of bidder $i$ is denoted by $F_{i}$. Write $F=\prod_{i} F_{i}$. The winner determination rule

$$
w: F \rightarrow N
$$

decides for each profile $f=\left(f_{i}\right)_{i \in N}$ of actions in $F$ who the winner of the object is. The payment function

$$
p: F \rightarrow \mathbb{R}
$$

determines for each profile $f$ of actions in $F$ the amount $p(f)$ the winner $w(f)$ has to pay to the auctioneer. A triplet $(F, w, p)$ is an auction.

Strategic behavior Each bidder has a valuation $v_{i}$ for the item. Valuations are drawn from a non-degenerate interval $I$ and are assumed to be private information. Bidders have to decide in advance which action to choose for each valuation they might possibly have. Thus, a strategy of bidder $i$ is a function $s_{i}: I \rightarrow F_{i}$ stating that bidder $i$, when having valuation $v_{i} \in I$, will take action $s_{i}\left(v_{i}\right)$ in $F_{i}$. A vector $s=\left(s_{i}\right)_{i \in N}$ of strategies is called a strategy profile.m

A realization $v=\left(v_{i}\right)_{i \in N}$ of valuations defines an ex post game $(F, w, p, v)$ with action space $F_{i}$ for bidder $i$ and payoff function $u_{i}\left(v_{i}\right): F \rightarrow \mathbb{R}$ defined by

$$
u_{i}\left(v_{i}\right)(f):= \begin{cases}v_{i}-p(f) & \text { if } i=w(f) \\ 0 & \text { otherwise }\end{cases}
$$

Since the ex post game $(F, w, p, v)$ is a game in normal form, the classical definition of Nash equilibrium applies. An action profile $f=\left(f_{i}\right)_{i \in N}$ is a Nash equilibrium of the ex post game $(F, w, p, v)$ if for every bidder $i$ and every action $g_{i} \in F_{i}$ of that bidder it holds that

$$
u_{i}\left(v_{i}\right)(f) \geq u_{i}\left(v_{i}\right)\left(f \mid g_{i}\right)
$$

where $\left(f \mid g_{i}\right)$ denotes the action profile where bidder $i$ chooses $g_{i}$ and every other bidder $j$ chooses $f_{j}$. A strategy profile $s=\left(s_{i}\right)_{i \in N}$ is an ex post equilibrium of the auction $(F, w, p)$ if for every realization $v=\left(v_{i}\right)_{i \in N}$ of valuations the action profile $s(v):=\left(s_{i}\left(v_{i}\right)\right)_{i \in N}$ in $F$ is a Nash equilibrium of the ex post game $(F, w, p, v)$. 
In the same way other notions also carry over to the setting of an auction. A strategy $s_{i}$ of bidder $i$ is dominant if for every realization $v_{i}$ of the valuation of bidder $i$ and any profile $f$ of actions

$$
u_{i}\left(v_{i}\right)\left(f \mid s_{i}\left(v_{i}\right)\right) \geq u_{i}\left(v_{i}\right)\left(f \mid g_{i}\right)
$$

holds for any action $g_{i} \in F_{i}$. Given a strategy profile $s$, strategy $s_{i}$ is a best response for player $i$ to $s$ if

$$
u_{i}\left(v_{i}\right)(s(v)) \geq u_{i}\left(v_{i}\right)\left(s(v) \mid f_{i}\right)
$$

for any realization $v=\left(v_{i}\right)_{i \in N}$ of valuations and any action $f_{i} \in F_{i}$. Strategy $s_{i}$ is ex post individually rational if for every realization $v_{i}$ of the valuation of bidder $i$ and any profile $f$ of actions,

$$
u_{i}\left(v_{i}\right)\left(f \mid s_{i}\left(v_{i}\right)\right) \geq 0 .
$$

The auction $(F, w, p)$ itself is called ex post individually rational if every bidder has an ex post individually rational strategy in it. A strategy profile $s$ is called ex post efficient if for every realization $v=\left(v_{i}\right)_{i \in N}$ it holds that

$$
w(s(v)) \in \arg \max \left\{v_{i} \mid i \in N\right\} .
$$

Direct auction An auction $(F, w, p)$ is called direct if $F_{i}=I$ for each bidder $i$. In other words, the action a bidder has to take in the auction is to report a valuation (not necessarily his true valuation). Since in a direct auction it is clear what the action spaces are, we will simply write $(w, p)$ to denote such an auction. A straightforward but important observation is that any strategy profile $s$ in $(F, w, p)$ automatically induces a direct auction $(w \circ s, p \circ s)$.

A direct auction $(w, p)$ is called a Vickrey auction if for every profile $r=\left(r_{i}\right)_{i \in N}$ of reported valuations in $I^{N}$ it holds that

$$
w(r) \in \arg \max \left\{r_{i} \mid i \in N\right\} \quad \text { and } \quad p(r):=\max \left\{r_{i} \mid i \neq w(r)\right\}
$$

It is very well known that in a Vickrey auction bidding your valuation is a dominant strategy. If every bidder bids according to this strategy, the outcome is ex post efficient, and the winner pays an amount equal to the second-highest valuation.

Query auction In a query auction the auctioneer sequentially queries the bidders about specific aspects of their valuation. As an answer to the query ${ }^{4}$ a bidder can

\footnotetext{
${ }^{4}$ Each separate query by the auctioneer could be thought of as a round in the auction because the action taken by the queried bidder is, at least in our setting, supposed to be publicly observable.
} 
choose one out of a finite set of actions. During the course of the auction each bidder may be, and usually will be, queried more than once.The number of times a particular bidder is queried during the auction is not assumed to be bounded, and may potentially be infinite. Determination of winner and payment in a query auction are based exclusively on the actions taken by the bidders in response to the queries of the auctioneer. The auction ends as soon as both winner and payment are determined.

In the next chapter we will give formal definitions of a specific type of query auctions, namely bisection auctions. However, for the purposes of this chapter the verbal description of a general query auction given so far is sufficient.

\subsection{The theorem of Green and Laffont}

In the next section we will use the following version of the Theorem by Green and Laffont, adjusted to our (simple) context. This theorem shows under precisely which conditions a direct auction is a Vickrey auction (see Green and Laffont [20]).

Theorem 6.3.1. A direct auction $(w \circ s, p \circ s)$ is a Vickrey auction if the following three conditions hold

(a) the auction $(F, w, p)$ is ex post individually rational;

(b) the strategy profile $s$ is an ex post equilibrium of $(F, w, p)$;

(c) the strategy profile $s$ is ex post efficient in $(F, w, p)$.

Proof. Assume that (a), (b) and (c) hold. We will show that $(w \circ s, p \circ s)$ is a Vickrey auction. To this end, let $v=\left(v_{i}\right)_{i \in N}$ be a profile of valuations in $I^{N}$. Since $s$ is ex post efficient, we know that

$$
(w \circ s)(v)=w\left(s_{i}\left(v_{i}\right)_{i \in N}\right) \in \arg \max \left\{v_{i} \mid i \in N\right\} .
$$

So we only have to show that $(p \circ s)(v)=v_{s e c}$, where

$$
v_{\text {sec }}:=\max \left\{v_{i} \mid i \neq(w \circ s)(v)\right\} .
$$

Write $i^{*}:=(w \circ s)(v)$. Moreover, denote the profile of realizations of valuations $\left(\left(v_{j}\right)_{j \neq i^{*}}, r\right)$ by $(v \mid r)$, and the profile of actions $\left(s_{j}\left(v_{j}\right)_{j \neq i^{*}} \mid s_{i^{*}}(r)\right)$ by $s(v \mid r)$. The proof is in two steps.

Only actions whose effects can only be observed at the same moment in time by other bidders are usually considered to be taken in the same round. Rounds typically differ from each other in terms of the information available to bidders. In that sense each query could be counted as a round. In this paper though we deviate slightly from this standard interpretation. The order in which bidders are queried is usually fixed, and a round is a sequence of queries in which each bidder is queried exactly once. 
I. First we will show that $(p \circ s)(v) \leq v_{\text {sec }}$. To this end, take a valuation $r \in I$ with $r>v_{\text {sec }}$. We show that $(p \circ s)(v) \leq r$.

Since the strategy profile $s$ is an ex post equilibrium of $(F, w, p)$ we know that $s(v)$ is a Nash equilibrium in the ex post game $(F, w, p, v)$. Because $i^{*}=(w \circ s)(v)$, we know that

$$
u_{i^{*}}\left(v_{i^{*}}\right)(s(v \mid r)) \leq u_{i^{*}}\left(v_{i^{*}}\right)(s(v))=v_{i^{*}}-(p \circ s)(v) .
$$

Now suppose bidder $i^{*}$ happens to have valuation $r$. Since $r>v_{\text {sec }}$, ex post efficiency of the strategy profile $s$ in $(F, w, p)$ implies that $i^{*}=(w \circ s)(v \mid r)$. Moreover, since the strategy profile $s$ is an ex post equilibrium of $(F, w, p)$ we know that $s(v \mid r)$ is a Nash equilibrium in the ex post game $(F, w, p,(v \mid r))$. Hence, by ex post individual rationality

$$
u_{i^{*}}(r)(s(v \mid r))=r-p(s(v \mid r))=r-(p \circ s)(v \mid r) \geq 0 .
$$

The last inequality implies that $r \geq(p \circ s)(v \mid r)$.

Now, suppose that bidder $i^{*}$ chooses action $s_{i^{*}}(r)$ while having valuation $v_{i^{*}}$. Again, since $r>v_{s e c}$, ex post efficiency of the strategy profile $s$ in $(F, w, p)$ implies that $i^{*}=(w \circ s)(v \mid r)$. So

$$
u_{i^{*}}\left(v_{i^{*}}\right)(s(v \mid r))=v_{i^{*}}-(p \circ s)(v \mid r) \geq v_{i^{*}}-r
$$

where the inequality follows from the result that $r \geq(p \circ s)(v \mid r)$.

Combination of the inequalities (6.1) and (6.2) yields $v_{i^{*}}-(p \circ s)(v) \geq v_{i^{*}}-r$. Hence, $(p \circ s)(v) \leq r$.

II. Secondly we will show that $(p \circ s)(v) \geq v_{s e c}$. To this end, take an $r \in I$ with $r<v_{\text {sec }}$. We show that $(p \circ s)(v) \geq r$.

Suppose bidder $i^{*}$ happens to have valuation $r$. Since the strategy profile $s$ is ex post efficient in $(F, w, p)$ and $r<v_{\text {sec }}$, we know that $i^{*} \neq(w \circ s)(v \mid r)$. Hence

$$
u_{i^{*}}(r)(s(v \mid r))=0
$$

However, since $s(v \mid r)$ is a Nash equilibrium in $(F, w, p,(v \mid r))$, we also know that

$$
u_{i^{*}}(r)(s(v \mid r)) \geq u_{i^{*}}(r)((s(v))=r-(p \circ s)(v) .
$$

Combining equality (6.3) and inequality (6.4) yields that $(p \circ s)(v) \geq r$. 


\subsection{Efficient query equilibria are almost always infinite}

Suppose we are given a query auction $(F, w, p)$ together with an ex post equilibrium $s=\left(s_{i}\right)_{i \in N}$ in this auction. Such an equilibrium is called a query equilibrium. Let $Z$ be the set of valuations $v=\left(v_{i}\right)_{i \in N}$ for which in the action profile $s(v):=\left(s_{i}\left(v_{i}\right)\right)_{i \in N}$ the auctioneer asks a finite number of queries before the auction ends. We will assume that $Z$ is measurable, and that moreover $w \circ s$ is also measurable. When $Z$ has Lebesgue measure equal to zero, we say that the query equilibrium $s$ is almost always infinite. When $Z$ has Lebesgue measure larger than zero, we say that $s$ is sometimes finite.

Theorem 6.4.1. Let $s$ be a query equilibrium in $(F, w, p)$ and suppose that $s$ is sometimes finite. Then the corresponding direct auction $(w \circ s, p \circ s)$ is not a Vickrey auction.

Proof. Define

$$
O(Z):=\{(w(s(v)), p(s(v))) \mid v \in Z\} .
$$

Let $Z^{k}$ be the set of valuations $v \in Z$ for which the auction ends after $k$ queries given the profile of actions $s(v)$. Then the cardinality of the set

$$
O\left(Z^{k}\right):=\left\{\left(w(s(v)), p(s(v)) \mid v \in Z^{k}\right\}\right.
$$

is finite since each player only has a finite number of possible responses to each query and the determination of winner and payment is based exclusively on the responses of the bidders to the queries of the auctioneer. Thus $O(Z)=\cup_{k=1}^{\infty} O\left(Z^{k}\right)$ is a countable set.

Now suppose that the corresponding direct auction $(w \circ s, p \circ s)$ is a Vickrey auction. Note that

$$
O(Z)=\{((w \circ s)(v),(p \circ s)(v)) \mid v \in Z\}
$$

Define $Z_{i}:=\{v \in Z \mid(w \circ s)(v)=i\}$. Since $Z$ and $w \circ s$ are measurable by assumption, also each $Z_{i}$ is measurable. So, since the $Z_{i}$ 's partition $Z$ and the Lebesgue measure of $Z$ is larger than zero, we know that at least one $Z_{i}$ must have Lebesgue measure larger than zero as well. Take such a $Z_{i}$. Define for each $p^{*} \in \mathbb{R}$

$$
Z_{i}\left(p^{*}\right):=\left\{v \in Z_{i} \mid(p \circ s)(v)=p^{*}\right\} .
$$

Since $(w \circ s, p \circ s)$ is a Vickrey auction we know that each set $Z_{i}\left(p^{*}\right)$ is a subset of the set

$$
\left\{v \in I^{N} \mid \max \left\{v_{i} \mid i \neq(w \circ s)(v)\right\}=p^{*}\right\}
$$


which has Lebesgue measure zero. Thus, each $Z_{i}\left(p^{*}\right)$ itself is measurable and has Lebesgue measure zero. Hence, the set

$$
P_{i}:=\left\{p^{*} \mid Z_{i}\left(p^{*}\right) \neq \phi\right\}
$$

must be uncountable, because $Z_{i}=\cup_{p^{*} \in P_{i}} Z_{i}\left(p^{*}\right)$ and $Z_{i}$ has Lebesgue measure larger than zero. The set $O(Z)$ must have a cardinality that is at least as large as the cardinality of $P_{i}$ because $p^{*} \mapsto\left(i, p^{*}\right)$ is an injective function from $P_{i}$ to $O(Z)$, so $O(Z)$ is uncountable. This contradicts our earlier conclusion that $O(Z)$ is a countable set. Hence, the direct auction ( $w \circ s, p \circ s)$ cannot be a Vickrey auction.

Theorem 6.4.2. Any ex post efficient ex post equilibrium in an ex post individually rational query auction is almost always infinite.

Proof. Consider an ex post efficient ex post equilibrium $s$ in an ex post individually rational query auction $(F, w, p)$. Theorem 6.3 .1 states that the corresponding direct auction $(w \circ s, p \circ s)$ is a Vickrey auction. However, if the equilibrium were sometimes finite, Theorem 6.4.1 states that the corresponding direct auction $(w \circ s, p \circ s)$ is not a Vickrey auction. Hence, the equilibrium cannot be sometimes finite. Since $Z$ is measurable by assumption, the equilibrium must be almost always infinite.

\subsection{Conclusion}

We have shown that in a setting with continuous valuations, any ex post equilibrium in an ex post individually rational query auction that ends with positive probability in finite time will necessarily be inefficient. Thus any implementation of the Vickrey auction by means of a query auction in the continuous setting will necessarily have an infinite running time for almost all realizations of valuations, and is hence not a feasible option in any practical sense. 


\section{Chapter 7}

\section{General bisection auctions}

In this chapter we show that the result of Chapter 6 has application. We introduce a class of query auctions, called general bisection auctions, and show that any auction from this class is ex-post individually rational and has an ex post equilibrium. We show that these equilibrium is sometimes finite and inefficient. According to the result of the previous chapter inefficiency of these equilibria is inevitable. ${ }^{1}$

\subsection{Introduction}

In the previous chapter we have shown that, in a setting where bidders have continuous valuations, ex post efficiency -allocating the item to a bidder with the highest valuation - in an ex-post equilibrium of a query auction can only be obtained at the price of an infinite running time of the auction for almost all realizations of valuations. Still, ex-post equilibrium need not always exist, which would render our result useless. Here we show that for a very wide class of query auctions, namely so-called general bisection auctions, an ex-post equilibrium exists so that the result of Chapter 6 has application.

The general bisection mechanism works as follows. Suppose a single indivisible object is auctioned to a set $N=\{1, \ldots, n\}$ of players. The players have independent private valuations, $v_{i}$, drawn from a common continuous probability distribution with cumulative density $F(v)$, within the range $[\alpha, \beta)$. We assume quasi-linear utilities. Valuations of players are private information, i.e. each player knows only his own valuation but not the valuations of the others. Before the start of the auction there is a lottery that determines the order of the players. W.l.o.g. we assume that this ordering is $1 \prec 2 \prec 3 \prec \cdots \prec n-1 \prec n$.

\footnotetext{
${ }^{1}$ The results of this chapter were first presented in Grigorieva et al. [23].
} 
The auction runs for an a priori indefinite number of rounds. In each round there is a specific payment $P$ to be made by a bidder if he wins in this round. In every round there is also a query price $Q$ which is higher than the current payment and an upper bound $H$ on future payments that is higher than the query price. Initially the payment and the upper bound are set as $P=\alpha$ and $H=\beta$, and all bidders are active. In every round the auctioneer asks the bidders that are active in that round whether they would be willing to pay the query price. Bidders are queried openly in increasing order.

If only one bidder is willing to pay the query price, he becomes the winner of the auction. He has to pay the current payment (not the query price). If more than one bidder is willing to pay the query price, the auction proceeds into the next round. Only those bidders who agreed to pay the query price stay active. The query price becomes the payment, and the new query price is raised to a level strictly above the old query price, but still below the upper bound. If no bidder is willing to pay the query price all bidders stay active, the payment stays the same, the old query price becomes the new upper bound, and the new query price is set between the new payment and the new upper bound. In case no winner is found, i.e., should the auction run indefinitely, then among the bidders who are still active the one with highest ranking wins and he pays the lowest price that is still higher than or equal to any of the payments that were announced while the auction was running.

Effectively a general bisection auction is a variation of the bisection auction presented in Chapter 2, the main two differences being that the auction proposed here may last indefinitely, and that it stops as soon as the winner is found. The bisection auction in Chapter 2 was designed to handle the situation in which bidders have discrete valuations. The present definition of a general bisection auction is specifically designed to handle continuous valuations.

In the remaining part of this chapter we focus on the strategic analysis of the proposed auctions and show that each general bisection auction has an ex post equilibrium. Given a general bisection auction we construct a specific equilibrium, called the bluff equilibrium, for that auction. The bluff equilibrium requires each bidder to act as follows. When there still is an active bidder with a lower rank in the ordering, the bidder stays in the auction until the query price exceeds his valuation. As soon as he becomes the active bidder with the lowest rank, he stays in the auction until the payment exceeds his valuation (effectively a bluff since he will say yes to a query price exceeding his valuation). We show that this strategy is ex post individually rational, and that the resulting profile where each bidder uses this strategy is an ex post equilibrium. Moreover we show that these equilibrium 
is sometimes finite, and even, under a mild assumption (namely that the price in the auction can in principle be driven up to exceed any possible valuation of any bidder $)^{2}$, has a running time that is finite for all realizations of valuations. A formal proof requires an exact framework, which we present in the next section.

\subsection{Formal representation of a general bisection auction}

A general bisection auction in the form described above is specifically designed to handle the case in which valuations are drawn from an interval $I=[\alpha, \beta)$. We will however first give a formal description of a general bisection auction that is free from any reference to valuations of the bidders.

We represent a general bisection auction as an extensive form game on a complete binary decision tree. We will describe this tree first and subsequently discuss the winner determination rule $W$ and the payment scheme $P$ to give a complete description of the auction.

We provide a complete description of the game tree. This tree is the same for the entire class of general bisection auctions. The nodes of the tree are given in (1) and the directed edges are defined in (2). The game board has perfect information, meaning that each node in the tree will be a decision node for one of the players. Hence information sets are obsolete.

The set of players that can participate in the game is $N=\{1, \ldots, n\}$. The response set for each player in every query node is $R=\{y e s, n o\}$. This reflects the fact that in each round of the auction each player will be faced with a binary query regarding his valuation. The precise nature of this query is explained in the next subsection.

(1) A node $a$ in the game tree is represented by the history of responses players have to give in order to reach this particular node. Formally, $a=\left(a_{k}\right)_{k=1}^{r}$ with $r \in \mathbb{N}$ where $a_{k}=\left(a_{k, i}\right)_{i=1}^{n}$ for $k<r$ and $a_{r}=\left(a_{r, i}\right)_{i=1}^{j}$ for some $j \leq n$. Here $a_{k, i}$ is a particular response in the set $R$ chosen by player $i$ in round $k$.

The length of a node is defined as $l(a)=(r-1) n+j$. The initial node $a_{0}=()$ has length zero. This node corresponds to the first round where the first player has to respond. The nodes with length larger than or equal to $(r-1) n$ but less than $r n$ correspond to round $r$, where the nodes with length $(r-1) n$ are referred to as the start of round $r$. The set of nodes corresponding to round $r$ is denoted by $X_{r}$. For a node $a \in X_{r}$, the node $a^{*} \in X_{r}$ is the node of length $(r-1) n$ for which $a_{k, i}^{*}=a_{k, i}$

\footnotetext{
${ }^{2}$ This is an assumption that is met by all existing auctions.
} 
for all $k<r$ and all $i \in N$.

A node $a$ that has a length of $l(a)=(r-1) n+j-1$ for some $r>0$ is a decision node of player $j$ in round $r{ }^{3}$ Let $D_{j}$ denote the collection of all decision nodes of player $j$ and $D_{j r}$ denote the collection of player $j$ 's decision nodes in round $r$. A predecessor of a decision node $a$ of player $j$ is a node from $D_{j}$ that player $j$ encounters when moving from the initial node $a_{0}$ to node $a$. We denote by $a(k) \in D_{j k}$ the predecessor of $a$ in round $k$. Conversely, $a$ is a successor of $a(k)$.

(2) There is a directed edge from node $a$ to node $b$ if $l(b)=l(a)+1$, and for all $j$ and $k$ for which $a_{k, j}$ is defined, $a_{k, j}=b_{k, j}$. So, there is an edge between two nodes if in the second node one player has given an additional response in comparison with the first node.

The next ingredient of the description of the game is the determination of the winner of the item and the specification of payments. As we allow general bisection auctions to last indefinitely, we will define the winner and the payment on infinite sequences of actions, which we call endnodes.

Thus, an endnode of the game is an infinite sequence $h=\left(h^{r, i}\right)_{r \in \mathbb{N}, i \in N}$ of nodes in the game tree such that (1) $h^{1,1}=a_{0}$ (the initial node is the first element of this sequence), and (2) there is an edge from node $h^{r, i}$ to node $h^{r, i+1}$ for any $i<n$, and from node $h^{r, n}$ to node $h^{r+1,1}$ for any $r \in \mathbb{N}$.

Each endnode $\left(h^{r, i}\right)_{r \in \mathbb{N}, i \in N}$ may be viewed as a history of infinite length such that its upper part of length $(r-1) n+i-1$ coincides with node $h^{r, i}$. Thus, the set of all possible endnodes of the game is order isomorphic to the set $2^{\mathbb{N}}$.

For an endnode $h=\left(h^{r, i}\right)_{r \in \mathbb{N}, i \in N}$, we denote by $A(h)$ the set of players who remain active throughout the play, that is $A(h)=\cap_{r} A\left(h^{r, 1}\right)$, where for a node $a \in X_{1}$ we define the set of active players by $A(a):=N$ and for a node $a \in X_{r+1}$ for some $r \geq 1$ we define the set $A(a)$ of active players iteratively by

$$
A(a):= \begin{cases}A(a(r)) & \text { if } a_{r, i}=\text { no } \forall i \in A(a(r)) ; \\ \left\{i \in A(a(r)): a_{r, i}=y e s\right\} & \text { otherwise. }\end{cases}
$$

Notice that $|A(h)| \geq 1$ will always hold. The winner of the game in endnode $h$ is

$$
W(h):=\max \{i \mid i \in A(h)\} .
$$

The difference between different general bisection auctions is in the payment rule. Suppose that $I=[\alpha, \beta)$. The price the winner pays depends on when it

\footnotetext{
${ }^{3}$ Notice that this implies that the bidders are queried according to the fixed ordering $1 \prec 2 \prec$ $3 \prec \cdots \prec n-1 \prec n$.
} 
became known that he is the winner. We will first provide a recursive description of the way the payment rule is constructed.

First we associate with each node $a \in X_{r}$ of length $(r-1) n$ a current price interval $[P(a), H(a))$ and a query price $Q(a)$ in the interior of this half-open interval. The query price $Q(a)$ bisects the current interval $[P(a), H(a))$ into two intervals $[P(a), Q(a))$ and $[Q(a), H(a))$ of smaller size. For this reason we call this auction a general bisection auction.

Once the game has reached node $a \in X_{r}$, the price $P\left(a^{*}\right)$ is the minimum amount the winner, whoever it may be, will have to pay, regardless of what happens from now on. In the same way $H\left(a^{*}\right)$ is a hard upper bound on the payment of the winner. The query in round $r$ associated with the query price $Q\left(a^{*}\right)$ is

Is your valuation larger than or equal to the query price $Q\left(a^{*}\right)$ ?

The answer to this query is an element of the response set $R=\{y e s, n o\}$, and only the responses of players that are currently active (in round $r$ that is) can influence the outcome of the auction.

Formally $P\left(a_{0}\right):=\alpha$ and $H\left(a_{0}\right):=\beta$, and $Q\left(a_{0}\right)$ is an element of the interval $\left(P\left(a_{0}\right), H\left(a_{0}\right)\right)$. For a node $a \in X_{r}$ of length $(r-1) n$ with $r>1$ we recursively define

$$
P(a):= \begin{cases}P(a(r-1)) & \text { if }\left|\left\{i \in A(a(r-1)): a_{r-1, i}=y e s\right\}\right| \leq 1 \\ Q(a(r-1)) & \text { otherwise. }\end{cases}
$$

and

$$
H(a):= \begin{cases}Q(a(r-1)) & \text { if }\left|\left\{i \in A(a(r-1)): a_{r-1, i}=y e s\right\}\right| \leq 1 \\ H(a(r-1)) & \text { otherwise. }\end{cases}
$$

Finally, we again choose $Q(a)$ in the interval $(P(a), H(a))$.

Now, for an endnode $h=\left(h^{r, i}\right)_{r \in \mathbb{N}, i \in N}$ with $|A(h)|=1$ we define the running time of the auction by $T(h)=\min \left\{r \in \mathbb{N}:\left|A\left(h^{r, 1}\right)\right|=1\right\}$. Otherwise we define the running time by $T(h)=\infty$. The price the winner in endnode $h$ pays is

$$
P(h):=\sup \left\{P\left(h^{r, 1}\right) \mid r \leq T(h)\right\} .
$$

All other players pay zero. The resulting payoff in endnode $h$ for player $j$ having valuation $v_{j}$ is given by

$$
U_{j}\left(v_{j}\right)(h):= \begin{cases}v_{j}-P(h) & \text { if } j=W(h) \\ 0 & \text { otherwise. }\end{cases}
$$


This completes the description of a general bisection auction in its representation as a query auction. Notice that effectively a general bisection auction is completely characterized by the choices of $\alpha$ and $\beta$, and the choices of the query price $Q(a)$ for every node $a$ that has a length $(r-1) n$ for some $r \geq 1$. The price bounds $P(a)$ and $H(a)$ as well as winner determination and payment specification are uniquely determined by the choices of $\alpha, \beta$, and the queries $Q(a)$.

One-shot representation of general bisection auctions A general bisection auction is a query auction, meaning that the auction has multiple rounds and in each round the players can give several (two in this case) responses to the queries of the auctioneer. We will briefly discuss how the one-shot representation $(F, w, p)$ of such an auction looks like in the terminology of Section 6.2.

A plan of action of player $j$ is a function $f_{j}$ that assigns to each decision node $a \in D_{j}$ a response $f_{j}(a)$ in $R$. The action set $F_{j}$ is the collection of all plans of action of player $j$. For the profile of plans of action $f=\left(f_{i}\right)_{i \in N}$ in $F:=\prod_{i} F_{i}$ the winner $w(f)$ and payment $p(f)$ are now defined as follows. The realization of $f$ is the endnode $h=\left(h^{r, i}\right)_{r \in \mathbb{N}, i \in N}$ where $h^{1,1}=a_{0}, h^{r, i+1}=\left(h^{r, i}, f_{i}\left(h^{r, i}\right)\right)$ for any $i<n$ and $h^{r+1,1}=\left(h^{r, n}, f_{n}\left(h^{r, n}\right)\right)$ for any $r \in \mathbb{N}$. Then $w(f):=W(h)$ and $p(f):=P(h)$. Automatically $u_{j}\left(v_{j}\right)(f)=U_{j}\left(v_{j}\right)(h)$.

This auction will be ex post individually rational as long as valuations are larger than or equal to $\alpha$. Indeed, in this case always saying no guarantees a player a non-negative payoff. If a player does so he can win only if all other players also keep on saying no, in which case the payment for the winner is $\alpha$.

\subsection{Ex post equilibrium of a general bisection auction}

In this section we will introduce for each general bisection auction a strategy profile that constitutes an individually rational ex-post Nash equilibrium in the given general bisection auction. We will also show that in equilibrium there is a set of valuations whose Lebesgue measure is larger than zero for which the auction ends in finite time. Consequently, in equilibrium, the allocation is not ex post efficient.

Consider the general bisection auction. Define the set of players who have ranking less than $j$ and are active in node $a \in D_{j}$ by

$$
A_{j}(a):=\{i \in A(a): i<j\} .
$$

These are the players whose decisions in the current round are observable for player $j$ when he has to make a decision in node $a$. Let

$$
D_{j}^{1}:=\cup_{r}\left\{a \in D_{j r} \mid \exists i \in A_{j}(a): a_{r, i}=y e s\right\}
$$


be the set of decision nodes of player $j$ such that there is at least one active predecessor of player $j$ whose action in the current round was yes. Thus, $D_{j}^{2}:=D_{j} \backslash D_{j}^{1}$ is the set of decision nodes of player $j$ such that all active predecessors of player $j$ took decision no.

As before, a strategy for player $j$ in a general bisection auction is a function $s_{j}$ that assigns to each possible valuation $v_{j} \in[\alpha, \beta)$ a plan of action $s_{j}\left(v_{j}\right)$ in $F_{j}$. Thus, for each decision node $a \in D_{j}$ of player $j, s_{j}\left(v_{j}\right)$ specifies a response $s_{j}\left(v_{j}\right)(a)$ in $R$.

Definition 7.3.1. Let $v_{j}$ be a valuation of player $j$ and let $a \in D_{j}$ be a decision node of player $j$. The bluff strategy $b_{j}$ of player $j$ is defined by

$$
b_{j}\left(v_{j}\right)(a):= \begin{cases}y e s & \text { if } a \in D_{j}^{1} \text { and } Q\left(a^{*}\right) \leq v_{j} \\ y e s & \text { if } a \in D_{j}^{2} \text { and } P\left(a^{*}\right) \leq v_{j} \\ n o & \text { otherwise. }\end{cases}
$$

This strategy has a bluffing component with regard to the query "Is your valuation larger than the current query price?". Indeed, in $D_{j}^{1}$ player $j$ compares his valuation $v_{j}$ with the current query price and in any node from $D_{j}^{2}$ with the current payment. So in nodes from $D_{j}^{2}$ when his valuation is larger than the payment even if it is smaller than the query he replies yes and thus deceives the auctioneer by pretending to have higher valuation than he really has. Therefore one can think of nodes from $D_{j}^{1}$ as truthful nodes and nodes from $D_{j}^{2}$ as bluff nodes.

To show that the profile $b=\left(b_{i}\right)_{i \in N}$ of bluff strategies constitutes an ex post individually rational ex post equilibrium we need the following results.

We say that a node from $D_{j}^{2}$ is in $M D_{j}^{2}$ if none of the predecessors of this node is in $D_{j}^{2}$. We analyze what happens in such a node when a player uses his bluff strategy.

Lemma 7.3.2. Suppose player $j$ has valuation $v_{j}$ and follows the plan of action $b_{j}\left(v_{j}\right)$. If a node $a$ is an element of $M D_{j}^{2}$ and if player $j$ is active in this node, then $b_{j}\left(v_{j}\right)(a)=$ yes.

Proof. Let $r$ be the round to which $a$ belongs. If $r=1$, then $P(a)=\alpha$, thus $v_{j} \geq P(a)$ and $j$ says yes. If $r>1$, by definition of $M D_{j}^{2}$ there was an active predecessor in the previous round who said yes. Since $j$ is still active, $j$ also said yes. Hence $v_{j} \geq Q(a(r-1))=P(a)$.

As a consequence after round $r$ player $j$ either is the winner (in case no other player who is active in round $r$ and has ranking larger than $j$ says yes) or he is 
active in round $r+1$ (otherwise). In any case all players with ranking less than $j$ are nonactive from then on.

Lemma 7.3.3. Suppose player $j$ follows his bluff strategy, and a is a decision node of player $j$ in round $r$. Suppose that player $j$ is active in a and says no for the first time. Then as long as player $j$ will stay active, his actions will be no.

Proof. If $a \in D_{j}^{1}$, then player $j$ is not active in any future round. Suppose then $a \in D_{j}^{2}$, then $v_{j}<P(a)<Q(a)$. This relation remains valid in any successor node of $a$.

Equivalently this lemma states that, if player $j$ follows his bluff strategy and says yes in node $a$ where he is active, then all previous actions of him were yes as well.

Proposition 7.3.4. A bluff strategy is ex post individually rational. Hence, as said before, general bisection auctions are ex post individually rational.

Proof. Suppose that player $j$ follows his bluff strategy and due to the plans of action chosen by the other players endnode $h$ is realized. If $j \neq W(h)$ then $u_{j}\left(v_{j}\right)(h)=0$. So, suppose that $j=W(h)$. We consider two cases. Case 1: if $T(h)<\infty$. Let $a$ be the decision node of player $j$ in round $T(h)$. Then all players in $A_{j}(a)$ said no, while player $j$ said yes. Therefore $v_{j} \geq P\left(a^{*}\right)$, and since $P\left(a^{*}\right)$ is the price to be paid by $j$, he has a non-negative payoff. Case 2: if $T(h)=\infty$. Since $j=W(h)$, Lemma 7.3.3 implies that player $j$ said yes in every round. Then, by definition of the bluff strategy, $P\left(h^{r, 1}\right) \leq v_{j}$ for every $r$. Hence, also $P(h) \leq v_{j}$.

Theorem 7.3.5. The strategy profile $b=\left(b_{i}\right)_{i \in N}$ is an ex post Nash equilibrium.

Proof. Let $\left(v_{i}\right)_{i \in N}$ be a realization of valuations and $f_{j} \in F_{j}$ be a plan of action of player $j$. Let $a$ be the first decision node at which player $j$ following $f_{j}$ deviates from $b_{j}\left(v_{j}\right)$. In case player $j$ is not active in node $a$ both $b_{j}\left(v_{j}\right)$ and $f_{j}$ yield payoff 0 and we are done. So suppose that player $j$ is active in node $a$. We consider two cases.

Case 1. In case $a \in D_{j}^{1}$. If $f_{j}(a)=n o$ and $b_{j}\left(v_{j}\right)(a)=y e s$, the payoff of playing $f_{j}$ is 0 , while according to Proposition 7.3.4 the payoff of playing $b_{j}\left(v_{j}\right)$ is at least 0 . Consider the case where $f_{j}(a)=$ yes and $b_{j}\left(v_{j}\right)(a)=$ no. When player $j$ says yes in $a$, there are at least two players who say yes in the current round by definition of $D_{j}^{1}$. So, the winning payment will be at least $Q\left(a^{*}\right)$. Further, $v_{j}<Q\left(a^{*}\right)$ because 
$b_{j}\left(v_{j}\right)(a)=n o$. Hence, the payoff of playing $f_{j}$ is non-positive while the payoff of playing $b_{j}\left(v_{j}\right)$ is 0 .

Case 2. In case $a \in D_{j}^{2}$. If $f_{j}(a)=$ yes and $b_{j}\left(v_{j}\right)(a)=n o$ we know that $P\left(a^{*}\right)>v_{j}$. Since the payment of the winner is at least $P\left(a^{*}\right)$, playing $f_{j}$ has nonpositive payoff, while playing $b_{j}\left(v_{j}\right)$ guarantees non-negative payoff. Consider the case where $f_{j}(a)=n o$ and $b_{j}\left(v_{j}\right)(a)=$ yes. Suppose that $b(v)=b_{i}\left(v_{i}\right)_{i \in N}$ is such that all successors of $j$ say no if $j$ says yes. Then, following $b_{j}\left(v_{j}\right)$ player $j$ wins at price $P\left(a^{*}\right)$ while following $f_{j}$ he might win at price at least $P\left(a^{*}\right)$. Now suppose that there is a successor $i$ of $j$ that plays yes if player $j$ says yes. Since player $i$ plays according to $b_{i}\left(v_{i}\right)$, he will also say yes when player $j$ switches to no. But then the payoff of playing $f_{j}$ would be 0 while the payoff of playing $b_{j}\left(v_{j}\right)$ is non-negative.

The following example shows that bidders that do not have the highest ranking do not have a dominant strategy. Hence the previous result cannot be strengthened much further. It can be shown that the bluff strategy is a dominant strategy for bidder $n$.

Example Consider a game with two players and suppose that player 2 has the following strategy: if in the first round player 1 says yes then play yes in the first round and no in all other rounds; otherwise play no in all rounds. Then any best response of player 1 against this strategy chooses no in the first round (and yes in some later round) whenever the valuation of player 1 is strictly larger than zero. Now, consider another strategy of player 2: if in the first round player 1 says yes then play no in all rounds; otherwise play yes in the first round and no in all other rounds. In this case any best response of player 1 against this strategy chooses yes in the first round (in decision node $a_{0}$ ) whenever the valuation of player 1 is strictly larger than zero. It follows that there is no strategy of player 1 which is a best response against both strategies of player 2 . Consequently player 1 does not have a dominant strategy.

Theorem 7.3.6. The strategy profile $b=\left(b_{i}\right)_{i \in N}$ is sometimes finite. Consequently, the allocation under $b$ is not ex post efficient.

Proof. Consider the set $V$ of realizations $v=\left(v_{i}\right)_{i \in N}$ of valuations for which $\alpha \leq v_{i}<Q\left(a_{0}\right)$ for all $i \in N$. Then for each $v \in V$ in round 1 bidder 1 says yes and all other bidders say no. Thus, the auction ends after this first round, and the Lebesgue measure of the set of valuations for which the auction ends after one round is at least $\left(Q\left(a_{0}\right)-\alpha\right)^{n}>0$. Consequently, by Theorems 6.4.2, 7.3.4, and 7.3.5, the allocation under $b$ is not ex post efficient. 
As in the above proof, consider the set $V$ of realizations $v=\left(v_{i}\right)_{i \in N}$ of valuations for which $\alpha \leq v_{i}<Q\left(a_{0}\right)$ for all $i \in N$. A direct way to conclude that allocation under $b$ is not ex post efficient is via the observation that for each $v \in V$ bidder 1 wins the item (for a price of $\alpha$ ). So, if we take for example $v_{1}=\alpha$, and $v_{i}=\frac{Q\left(a_{0}\right)+\alpha}{2}$ for all $i \neq 1$, then the allocation is not ex post efficient.

\subsection{Finite running time}

Not every general bisection auction will have a finite running time under the bluff equilibrium for any realization of valuations. If we take for example $\alpha=0, \beta=1$, and for each $a \in X_{r}$

$$
Q\left(a^{*}\right)=\left(1-\frac{1}{(r+1)^{2}}\right) P\left(a^{*}\right)+\frac{1}{(r+1)^{2}} H\left(a^{*}\right)
$$

we get a general bisection auction for which for each endnode $h$ we have that $P(h) \leq$ $\frac{3}{4} .{ }^{4}$ It is clear that the running time in equilibrium is not finite as soon as at least two players have a valuation larger than $\frac{3}{4}$. In order to exclude such pathological cases, consider the quantity

$$
P^{*}:=\sup \{P(a) \mid a \text { is of length }(r-1) n \text { for some } r \in \mathbb{N}\}
$$

This quantity is the price the winner has to pay in a general bisection auction when in any round there are at least two players who say yes to their query in that round.

We say that a general bisection auction is regular if $P^{*}=\beta$. Regularity implies for example that the price can in principle be driven up by the bidding process to a level where a bidder makes a loss if he becomes the winner.

Lemma 7.4.1. Given the realization of valuations $\left(v_{i}\right)_{i \in N}$ suppose that in a regular general bisection auction player $j$ follows his plan of action $b_{j}\left(v_{j}\right)$ while all other players follow $s_{-j}\left(v_{-j}\right)$-the profile of plans of action corresponding to an arbitrary profile of strategies $s_{-j}$. Let $h$ be the realization of the game if the profile $\left(b_{j}\left(v_{j}\right), s_{-j}\left(v_{-j}\right)\right)$ is played. If $j=W(h)$ then $T(h)<\infty$.

Proof. Suppose that $T(h)=\infty$. It implies that $|A(h)|>1$ and, by definition of $W(h)$, for all $i \in A(h)$ it holds that $i \leq j$. First we argue that no player in $A(h)$ can say yes indefinitely. If a player in $A(h)$ would say yes indefinitely, then, because $j=W(h)$, player $j$ must do so as well. However, since player $j$ follows his

\footnotetext{
${ }^{4}$ Use the fact that $\frac{1}{(r+1)^{2}} \leq \frac{1}{r(r+1)}=\frac{1}{r}-\frac{1}{r+1}$ for every $r \geq 2$.
} 
bluff strategy, this implies that $\beta=P^{*} \leq v_{j}$ by the regularity of the auction. This contradicts the assumption that $v_{j}$ is drawn from $[\alpha, \beta)$.

Consider the round in history $h$ where for the first time a player $i$ from $A(h)$ says no. Let $a$ be the decision node that player $j$ reaches in this round. If any other player from $A_{j}(a)$ says yes in this round then $i$ is not active in the next round, which contradicts the fact that $i \in A(h)$. If all players from $A_{j}(a)$ say no in this round then, according to Lemma 7.3.2, player $j$ says yes and, again, $i$ is not active anymore after this round. Since $T(h)=\infty$, this means that any player from $A(h)$ says yes indefinitely. Contradiction. Hence, we conclude that $T(h)<\infty$.

As an immediate consequence of Lemma 7.4.1, the bluff equilibrium of a regular general bisection auction guarantees a finite running time for any realization of valuations. Notice that this statement is stronger than just saying that we have a finite running time almost surely. Furthermore, from Lemma 7.3.2 it immediately follows that, when every bidder plays according to his bluff strategy, in any round of the auction there is at least one player that says yes. Thus we have established the following Theorem.

Theorem 7.4.2. In a regular general bisection auction the profile of bluff strategies has a finite running time for any realization of valuations. Moreover, the query price increases from round to round up to the moment where the winner is found.

\subsection{Conclusion}

We have shown that the inefficiency result of Chapter 6 applies to a wide class of query auctions, in particular general bisection auctions. According to this result inefficiency of the bluff equilibrium of a general bisection auction is inevitable. In the next chapter we will analyze exactly how (in)efficient bluff equilibrium may be. In particular we show that within the class of general bisection auctions any level of approximate efficiency can be achieved with a finite running time for all realizations of valuations. 



\section{Chapter 8}

\section{The family of $c$-bisection auctions: efficiency and running time}

In this chapter we introduce a special type of general bisection auction, the $c$ bisection auction, and analyze its performance. We discuss the running time and the efficiency in the ex-post equilibrium of the auction. We show that by changing the parameter $c$ of the auction we can trade off efficiency against running time and achieve any desirable level of efficiency with finite running time. Moreover, we show that the auction that gives the desired level of efficiency in expectation takes the same number of rounds for any number of players. ${ }^{1}$

\subsection{Introduction}

In Chapter 6 we studied the uses and limitations of query auctions regarding the objective of economic efficiency maximization. In particular, we proved that in a setting with continuous valuations any ex-post equilibrium in an ex-post individual rational query auction that ends with positive probability after a finite number of queries, can not be fully efficient. This result implies that in the setting of continuous valuations full efficiency can only be achieved at the expense of an infinite running time of a query auction for almost all realizations of valuations. So the question that arises is: what running time can be achieved if we are satisfied with a particular level of approximate efficiency.

Thus, in this chapter we are concerned with the trade-off between running time

\footnotetext{
${ }^{1}$ The results of this chapter were first presented in Grigorieva et al. [22].
} 
and allocative efficiency in the proposed general bisection auction. In particular, we consider a special type of a general bisection auction, the $c$-bisection auction. The auction is characterized by a parameter $c$ which, together with the distribution from which valuations of players are drawn, determines a sequence of query prices. The $c$-bisection auction has an ex-post equilibrium, called the bluff equilibrium, and under this equilibrium the auction ends in finite time, regardless of the realization of players' valuations. Due to the result mentioned above we know that inefficiency of the bluff equilibrium for some realizations of valuations is inevitable.

Here we discuss in detail the performance of the $c$-bisection auction under the bluff equilibrium. In particular, we study how the choice of parameter $c$ and the number of participating players affect the running time of the auction and its (in)efficiency.

First, we investigate the running time of the auction according to two measures, namely the expected number of rounds and the expected number of queries performed in the auction. ${ }^{2}$ For both measures we derive first a recursive formula and give then an upper bound for the function defined by this formula. We prove that for a fixed $c$ the expected number of rounds is bounded by a function that is logarithmic in the number of players while the expected number of queries is bounded by a function that is linear in the number of players.

Second, we analyze the level of inefficiency of the auction. As measures of inefficiency we employ the probability of inefficient allocation and the expected loss of welfare. For the probability of inefficient allocation we derive a recursive formula and prove that it is not more than $c$ for any number of players. We show that when valuations are uniformly drawn from $[0,1)$ the expected loss of welfare is bounded from above by $c^{2}$ for any number of players. It means that by choosing the appropriate $c$, the minimum level of efficiency can be determined by the auctioneer before it is known how many players will participate in the auction. We also give a (more) precise estimate of the expected loss of welfare by using computer simulation.

Furthermore we show that for a fixed number of players there is a trade-off between efficiency and running time: for the increasing efficiency of the auction we have to pay by an increasing number of rounds and an increasing number of queries. By simulation it turns out that the trade-off curves, which show the relation between the expected number of rounds and the expected loss of welfare, constructed for different numbers of players coincide with each other. Thus, in expectation the number of rounds of the auction that obtains a desired level of efficiency is

\footnotetext{
${ }^{2}$ As a query we consider each separate question of the auctioneer to an active player. As a round we consider a sequence of queries in which each active player is asked to act exactly once.
} 
independent of the number of players.

Related literature The trade-off between the running time/communication complexity and the level of allocative efficiency of query auctions has been recently examined by some researchers. In particular, several issues concerning welfare of single-item limited revelation auctions have been considered. In David et al. [16] the issue is considered for English auctions when restricting queries to discrete levels. The authors analyze how the choice of query levels in the English auction affects the expected welfare and the expected duration (measured in terms of the number of leels that the query price has been raised through). Blumrosen et al. $[4,5]$ study the effect on welfare of a severe restriction of the amount of communication allowed in an auction. They consider the case where each bidder is only allowed to submit one of $k$ distinct bids, requiring communication of $\lg (k)$ bits. They show that for any prior over valuations, there exists an one-shot auction, called a priority game, that incurs a welfare loss (relative to an optimal unlimited communication auction, e.g. the Vickrey auction) which is bounded above by $O\left(\frac{1}{k^{2}}\right)$ for a fixed number of players. For the case of 2 players it is shown that a priority game is welfare-optimal among those 2-player mechanisms allowing at most $k$ distinct bids. In Blumrosen et al. [5] additionally a sequential mechanism is considered in which players split their bids to smaller messages and send them in alternating order with full knowledge of all previously revealed bids by other players. They show that such mechanisms can achieve better results, the additional gain in communication, however, is limited. Namely, for every sequential mechanism with total communication of $m$ bits there exists a one-shot mechanism that achieves at least the same expected welfare with communication not more than $n m$, where $n$ is the number of players.

\subsection{The $c$-bisection auction}

First, we repeat the rules of a general bisection auction (choosing description that suits the best the needs of this chapter) and then we introduce a specific type of it, the $c$-bisection auction.

Suppose a single indivisible object is auctioned to a set $N=\{1, \ldots, n\}$ of players. The players have independent private valuations, $v_{i}$, drawn from a common continuous probability distribution with cumulative density $F(v)$, within the range $[\alpha, \beta)$. We assume quasi-linear utilities. Valuations of players are private information, i.e. each player knows only his own valuation but not the valuations of the others. Before the start of the auction there is a lottery that determines the order of the players. W.l.o.g. we assume that this ordering is $1 \prec 2 \prec 3 \prec \cdots \prec n-1 \prec n$. A 
player with a lower ranking is called a predecessor. So e.g. player 5 is a predecessor of player 7 .

The auction runs for an a priori indefinite number of rounds. Each round is characterized by payment $p_{r}$, query price $q_{r}$, upper bound $u_{r}$ and a set of active players $A_{r}$. The payment specifies the price to be payed if a player wins in this round. The query price is used by the auctioneer to ask an active player whether his valuation is larger than or equal to the query price. Players are queried openly in increasing order, so that a player can observe the bids of his predecessors. In each round the query price $q_{r}$ chosen form the open interval $\left(p_{r}, u_{r}\right)$.

The initial set of active players is $A_{1}=N$. The auction starts with $p_{1}=\alpha$ and $u_{1}=\beta$ and $q_{1}$ is a point in $(\alpha, \beta)$. Given the current set $A_{r}$, the payment $p_{r}$, the query price $q_{r}$, the upper bound $u_{r}$ and the bids of players in round $r$ the characteristics of the next round $r+1$ are defined as follows. If all players submit a no bid they all remain active, i.e. $A_{r+1}=A_{r}$. The payment remains the same and the upper bound is set to the previous query price, i.e. $p_{r+1}=p_{r}$ and $u_{r+1}=q_{r}$. If at least two players submit a yes bid, all players that said yes remain active. The upper bound remains the same and the payment is set equal to the previous query price, i.e. $u_{r+1}=u_{r}$ and $p_{r+1}=q_{r}$. The new bounds determine a new query price $q_{r+1}$ in $\left(p_{r+1}, u_{r+1}\right)$. If only one player submits a yes bid the auction stops, this player wins the auction and pays $p_{r}$. If such a moment does not occur, i.e. at least two players remain always active, the winner is determined according to the order of players: among those players who remain active the player with the highest ranking wins. The price the winner pays is equal to the limit of the sequence of the payments that occurred in the subsequent rounds in the auction. Since the sequence of payments is increasing this limit is equal to the supremum of the payments.

In the special type of a general bisection auction called $c$-bisection auction, the query price is defined as follows. For any $c \in(0,1)$ and any continuous probability distribution with cumulative density $F(v)$ from which valuations of players are drawn, in any round $r$, given the payment $p_{r}$ and the upper bound $u_{r}$, the query price $q_{r}$ is chosen such that

$$
\frac{F\left(q_{r}\right)-F\left(p_{r}\right)}{F\left(u_{r}\right)-F\left(p_{r}\right)}=c
$$

i.e. interval $\left[p_{r}, q_{r}\right)$ contains a fraction $c$ of the measure of $\left[p_{r}, u_{r}\right)$. For example, for the uniform distribution the query price bisects the interval $\left[p_{r}, u_{r}\right)$ in fractions $c$ and $1-c$ so that $q_{r}=p_{r}+c\left(u_{r}-p_{r}\right)$.

The following strategy, called the bluff strategy, constitutes a symmetric ex-post 
equilibrium in the $c$-bisection auction (this result is proven in Chapter 7). Under the bluff strategy an active player $i$ having valuation $v_{i}$ says yes in round $r$ whenever:

1. $v_{i} \geq q_{r}$, or

2. $p_{r} \leq v_{i}<q_{r}$ and no active predecessor of him said yes in this round.

The following example illustrates how the $c$-bisection auction proceeds under the bluff equilibrium.

Example. Suppose five players with valuations uniformly distributed on $[0,1)$ participate in the $c$-bisection auction with $c$ equal to 0.5 . Suppose that according to the lottery the ordering of players is $A \prec B \prec C \prec D \prec E$. Players have the following private valuations respectively: $0.43,0.71,0.38,0.79$, and 0.86 . The auction proceeds as follows:

\begin{tabular}{llllccccc}
\hline Round & Payment & Query & Set of act. & Player & Player & Player & Player & Player \\
$r$ & $p_{r}$ & price $q_{r}$ & players $A_{r}$ & A & B & C & D & E \\
\hline 1 & 0 & 0.5 & ABCDE & yes & yes & no & yes & yes \\
2 & 0.5 & 0.75 & ABDE & no & yes & - & yes & yes \\
3 & 0.75 & 0.875 & BDE & - & no & - & yes & no \\
\hline
\end{tabular}

In the first round player $\mathrm{A}$, having no predecessor and valuation larger than $p_{1}$ says yes. Every other player, having predecessor A with yes decision, says yes iff his valuation is larger than $q_{1}=0.5$. All players except $\mathrm{C}$ say yes and therefore remain active. The payment and the query price increase to 0.5 and 0.75 , respectively. Since $v_{A}<p_{2}$ player A says no in the second round. Now player B has no predecessor with yes decision and since $v_{B}>p_{2}$ he says yes. Players $\mathrm{D}$ and $\mathrm{E}$ say yes since their valuations are larger than $q_{2}=0.75$. Again the payment and the query price increase. In the third round player B says no, player $D$, having now no predecessors with yes decision, says yes and player E says no. So there is only one yes decision meaning that the auction ends. Player D wins the auction and pays 0.75 .

Notice that the outcome in the example is not efficient - the winner is not the player with the highest valuation. But as we have already pointed out inefficiency for some realizations of valuations is inevitable. Later in the chapter we investigate how inefficient this auction is by analyzing the probability of inefficient allocation and the expected loss of welfare.

Probability distribution of player actions. In the remaining part of the chapter we focus on auction performance in expectation. In order to analyze the expected performance we need to know the probability of particular actions of players. 
Namely, we need to know the probability of saying yes and no by an active player under the bluff strategy.

Recall that in any round $r$ of the $c$-bisection auction the query price $q_{r}$ is determined in such a way that, given that the valuation of a player is in $\left[p_{r}, u_{r}\right)$, the probability that his valuation is in $\left[p_{r}, q_{r}\right)$ is equal to $c$. Write $i_{r}:=\min \left\{i \mid i \in A_{r}\right\}$ - among the active players in round $r$ the one with the lowest ranking; $j_{r}:=\min \{i \mid$ $\left.i \in A_{r}, i \neq i_{r}\right\}$ - among the active players in round $r$ the one with the second lowest ranking.

First let us observe that when player $i_{r}$ says no for the first time, player $j_{r}$ says yes with certainty. Indeed, in all previous rounds player $i_{r}$ said yes and since $j_{r}$ is active in round $r$ also he said yes in those rounds. Both the payment and the query price increased so that $p_{r}=q_{r-1}$. Since player $j_{r}$ follows the bluff strategy his previous yes decision implies that $v_{j_{r}} \geq q_{r-1}=p_{r}$. If in round $r$ player $i_{r}$ says $n o$, player $j_{r}$ is in the situation where he does not have any active predecessor with yes decision and therefore says yes whenever his valuation is not smaller than $p_{r}$, that is with certainty. It follows that after round $r$ player $i_{r}$ drops out so that $i_{r+1}=j_{r}$. Notice that in the equilibrium in every round $r$ either player $i_{r}$ or player $j_{r}$ (or both) say yes. It means that in the equilibrium only players with yes decision remain active. This in its turn implies that the upper bound always remains the same so that $u_{r}=\beta$ for any $r$, and the payment and the query price increase, so that $p_{r}=q_{r-1}$ for any $r>1$.

Second, we need to know the probability that player $i_{r}$ says yes in round $r$. Having no active predecessor player $i_{r}$ says yes iff $v_{i_{r}} \geq p_{r}$. Since $p_{1}=\alpha$ player $i_{1}$ in round 1 says yes with certainty. Now let us show that for any $r>1$ the probability that player $i_{r}$ says yes in round $r$ equals $1-c$. Regarding the identity of player $i_{r}$ there are two possibilities - either $i_{r}=i_{r-1}$ (happens if decision of $i_{r-1}$ was yes) or $i_{r}=j_{r-1}$ (happens if decision of $i_{r-1}$ was no and consequently decision of $j_{r-1}$ was yes). In both cases the decision of player $i_{r}$ in round $r-1$ was yes implying that $v_{i_{r}} \geq p_{r-1}$. Thus, $P\left(v_{i_{r}} \geq p_{r} \mid v_{i_{r}} \geq p_{r-1}\right)=P\left(v_{i_{r}} \geq q_{r-1} \mid v_{i_{r}} \geq p_{r-1}\right)=1-c$. The last equality holds because $q_{r-1}$ divides the interval $\left(p_{r-1}, \beta\right)$ exactly in such a way that this conditional probability is equal to $1-c$.

Further, we need to know the probability of saying yes in round $r$ for any player $i \neq i_{r}, i \in A_{r}$. We can distinguish two cases. First, consider the case where player $i$ says yes in round $r$. From the fact that $i \in A_{r}$ follows that player $i_{r}$ said yes in round $r-1$ and thus $v_{i} \geq q_{r-1}$. In round $r$ he says yes iff $v_{i} \geq q_{r}$. Thus, $P\left(v_{i} \geq q_{r} \mid v_{i} \geq q_{r-1}\right)=P\left(v_{i} \geq q_{r} \mid v_{i} \geq p_{r}\right)=1-c$. The last equality holds because $q_{r}$ divides the interval $\left(p_{r}, \beta\right)$ exactly in such a way that this conditional 
probability is equal to $1-c$. Secondly, consider the case where player $i_{r}$ says no in round $r$. As we described above player $j_{r}$ says yes with certainty. For any other player $i$ the situation is the same as in the previous case and thus also here player $i \in N \backslash\left\{i_{r}, j_{r}\right\}$ says yes with probability $1-c$.

Now notice that the analysis above was done without specifying the distribution function from which valuations of players are drawn. Due to the price setting rule of the $c$-fraction auction the obtained probability results hold regardless of the distribution function of valuations. Thus, in the remaining part of the chapter, namely in Section 8.4, for simplicity of argumentation we focus on the setting where valuations of players are independently drawn from the uniform distribution in $[0,1)$. Moreover, it could be seen from the analysis above that the probability of saying yes or no by an active player does not depend on the round. It enables us to derive recursive formulas (in the number of active players) for the expected number of rounds and the expected number of queries performed in the auction.

\subsection{Running time of the $c$-bisection auction}

In this section we investigate the expected running time of the $c$-bisection auction if the bluff strategies are played. We analyze two measures, namely the expected number of rounds and the expected number of queries performed in the auction before the winner is found. As a query we consider each separate question of the auctioneer to an active player. As a round we consider a sequence of queries in which each active player is asked to act exactly once. For both measures we derive first a recursive formula and give then an upper bound for the function defined by this formula.

\subsubsection{The expected number of rounds}

Let $e_{c}(k)$ be the expected number of rounds of the auction with $k$ active players, given that the decision of the active player with the lowest ranking is yes in the current round; and $e_{c}^{*}(k)$ be the expected number of rounds given that this decision is no. Consider round $r$ with $n$ active players and suppose that the decision of player $i_{r}$ in the current round is yes. The current round contributes 1 to $e_{c}(n)$. Now let us compute the expected number of remaining rounds. If all active players apart from player $i_{r}$ say no, the auction stops after this round. If $k$ (for some $1 \leq k \leq n-1$ ) active players apart from player $i_{r}$ say yes, then the auction continues with $k+1$ active players. The probability of this situation given the yes decision of player $i_{r}$ is $\left(\begin{array}{c}n-1 \\ k\end{array}\right)(1-c)^{k} c^{n-1-k}$ (since when player $i_{r}$ says yes all other players say yes and no 
with probabilities $1-c$ and $c$ respectively). In the next round player $i_{r+1}=i_{r}$ says yes or no with probabilities $1-c$ and $c$ respectively. Thus if $k$ active players apart from player $i_{r}$ say yes in the round $r$, the expected number of remaining rounds is equal to $(1-c) e_{c}(k+1)+c e_{c}^{*}(k+1)$. Hence, for any $n \geq 2$

$$
e_{c}(n)=1+\sum_{k=1}^{n-1}\left(\begin{array}{c}
n-1 \\
k
\end{array}\right)(1-c)^{k} c^{n-1-k}\left[(1-c) e_{c}(k+1)+c e_{c}^{*}(k+1)\right] \text {. }
$$

Now recall that if player $i_{r+1}$ says no player $j_{r+1}$ says yes with certainty, which causes player $i_{r+1}$ to drop out of the auction. Thus, $e_{c}^{*}(2)=1$ and $e_{c}^{*}(k+1)=e_{c}(k)$ for any $k>1$. These observations are used in Appendix 8.6 to rewrite the above recursive relation to

$$
\left[1-(1-c)^{n}\right] e_{c}(n)=1+(n-1)(1-c) c^{n-1}+\sum_{k=2}^{n-1}\left(\begin{array}{l}
n \\
k
\end{array}\right)(1-c)^{k} c^{n-k} e_{c}(k) .
$$

This formula is valid for any $n \geq 2$.

Now notice that since in the first round player $i_{1}$ says yes with certainty, the expected number of rounds of the auction of $n$ players is equal to $e_{c}(n)$. Thus using formula 8.2 we can compute the expected number of rounds in the auction of $n$ players. Plugging in $n=2$ yields $e_{c}(2)=\frac{1+c(1-c)}{c(2-c)}$. All other values can be determined recursively. Table 8.1 in Appendix 8.6 presents the computational results for different values of $c$ in the auction with up to 100 players (data is within an accuracy of 0.001). Figure 8.1(a) shows how for a fixed value of $c$ the expected number of rounds increases in the number of players who participate in the auction. Furthermore, Figure 8.1(b) demonstrates how for a fixed number of players the expected number of rounds decreases as $c$ increases.

Generally we show that the expected number of rounds of the auction is bounded from above by a function that is logarithmic in the number of players. To prove this, first we introduce several notations and lemmas.

Define $D_{n}=\prod_{k=1}^{n} \frac{1}{1-(1-c)^{k}}$ for any $n \geq 2$.

Also define $E_{2}=\frac{1+c(1-c)}{c(2-c)}$ and for any $n>2$

$$
E_{n}=1+(n-1)(1-c) c^{n-1}+\sum_{k=2}^{n-1}\left(\begin{array}{l}
n \\
k
\end{array}\right)(1-c)^{k} c^{n-k} E_{k}
$$




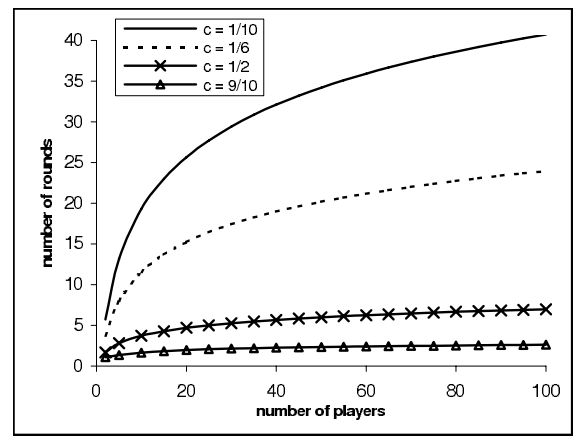

a)

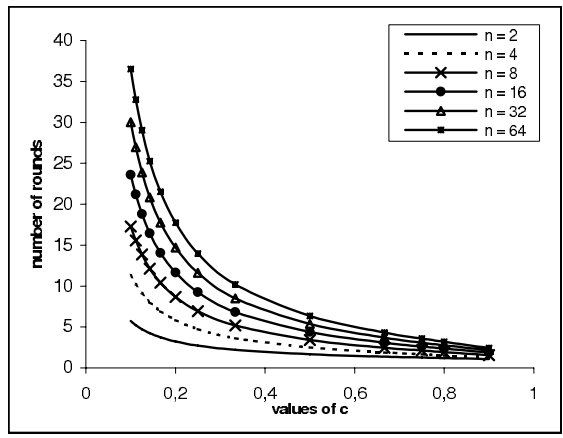

b)

Figure 8.1: The expected number of rounds (a) for different fixed values of $c$; (b) for different fixed numbers of players.

Lemma 8.3.1. For any $n \geq 2, e(n) \leq E_{n} \cdot D_{n}$.

Proof. The proof is by induction on $n$. The basis of the induction is trivial since $e_{c}(2)=E_{2}$ and $D_{2}>1$. Suppose that $e_{c}(k) \leq E_{k} \cdot D_{k}$ is true for any $2 \leq k \leq n-1$. Notice that $D_{n} \geq D_{n-1} \geq \ldots \geq D_{2}>1$. Thus, using the recursive formula for $e_{c}(n)$ and the induction hypothesis,

$$
\begin{aligned}
{\left[1-(1-c)^{n}\right] e_{c}(n) } & =1+(n-1)(1-c) c^{n-1}+\sum_{k=2}^{n-1}\left(\begin{array}{l}
n \\
k
\end{array}\right)(1-c)^{k} c^{n-k} e_{c}(k) \\
& \leq 1+(n-1)(1-c) c^{n-1}+\sum_{k=2}^{n-1}\left(\begin{array}{l}
n \\
k
\end{array}\right)(1-c)^{k} c^{n-k} E_{k} D_{k} \\
& \leq 1+(n-1)(1-c) c^{n-1}+\sum_{k=2}^{n-1}\left(\begin{array}{l}
n \\
k
\end{array}\right)(1-c)^{k} c^{n-k} E_{k} D_{n-1} \\
& \leq D_{n-1}\left[1+(n-1)(1-c) c^{n-1}+\sum_{k=2}^{n-1}\left(\begin{array}{l}
n \\
k
\end{array}\right)(1-c)^{k} c^{n-k} E_{k}\right] \\
& =E_{n} \cdot D_{n-1},
\end{aligned}
$$

which completes the proof. 
Now we find bounds on $D_{n}$ and $E_{n}$.

Lemma 8.3.2. For any $n \geq 2, D_{n} \leq e^{\frac{1-c}{c^{2}}}$.

Proof. It is enough to show that $\ln D_{n} \leq \frac{1-c}{c^{2}}$. Let us define $\lambda=\frac{1}{1-c}$. Notice that since $0<c<1$ it holds that $\lambda>1$.

We have

$$
\begin{aligned}
\ln D_{n} & =\ln \left(\prod_{k=1}^{n} \frac{\lambda^{k}}{\lambda^{k}-1}\right)=\sum_{k=1}^{n}\left[\ln \lambda^{k}-\ln \left(\lambda^{k}-1\right)\right] \leq \sum_{k=1}^{n}(\ln x)_{\mid x=\lambda^{k}-1}^{\prime} \\
& =\sum_{k=1}^{n} \frac{1}{\lambda^{k}-1} \leq \sum_{k=1}^{n} \frac{1}{\lambda^{k}-\lambda^{k-1}}=\frac{1}{\lambda-1} \sum_{k=1}^{n} \frac{1}{\lambda^{k-1}} \leq \frac{1}{\lambda-1} \sum_{k=0}^{\infty} \frac{1}{\lambda^{k}} \\
& =\frac{\lambda}{(\lambda-1)^{2}}=\frac{1-c}{c^{2}} .
\end{aligned}
$$

Lemma 8.3.3. For any $n \geq 2$ and any $c \leq \frac{1}{2}, E_{n} \leq 1+\log _{a} n$, with base $a=\frac{1}{1-c}$.

Proof. The proof is by induction on $n$. The basis of induction holds since $\frac{1+c(1-c)}{c(2-c)} \leq$ $\log _{a} 2+1$ for any $c \leq \frac{1}{2}$. Suppose $E_{k} \leq 1+\log _{a} k$ for any $2 \leq k \leq n-1$. Using the induction hypothesis,

$$
\begin{aligned}
E_{n} & =1+(n-1)(1-c) c^{n-1}+\sum_{k=2}^{n-1}\left(\begin{array}{l}
n \\
k
\end{array}\right)(1-c)^{k} c^{n-k} E_{k} \\
& \leq 1+(n-1)(1-c) c^{n-1}+\sum_{k=2}^{n-1}\left(\begin{array}{l}
n \\
k
\end{array}\right)(1-c)^{k} c^{n-k}\left(\log _{a} k+1\right) \\
& \leq 1+\sum_{k=2}^{n-1}\left(\begin{array}{l}
n \\
k
\end{array}\right)(1-c)^{k} c^{n-k} \log _{a} k+\sum_{k=1}^{n-1}\left(\begin{array}{l}
n \\
k
\end{array}\right)(1-c)^{k} c^{n-k} \\
& \leq 2+\sum_{k=2}^{n-1}\left(\begin{array}{l}
n \\
k
\end{array}\right)(1-c)^{k} c^{n-k} \log _{a} k .
\end{aligned}
$$

Since the logarithm with base $a=\frac{1}{1-c}$ is concave, we know that if $\lambda_{k} \geq 0$ and $\sum_{k=0}^{n} \lambda_{k}=1$ then

$$
\sum_{k=0}^{n} \lambda_{k} \log _{a}\left(x_{k}\right) \leq \log _{a}\left(\sum_{k=0}^{n} \lambda_{k} x_{k}\right) .
$$

So let us take $\lambda_{k}=\left(\begin{array}{c}n \\ k\end{array}\right)(1-c)^{k} c^{n-k}$ for all $k$ and take $x_{0}=x_{n}=1, x_{k}=k$ for any $1 \leq k \leq n-1$. Then 


$$
\begin{aligned}
E_{n} & \leq 2+\sum_{k=2}^{n-1}\left(\begin{array}{l}
n \\
k
\end{array}\right)(1-c)^{k} c^{n-k} \log _{a} k \\
& =2+\sum_{k=0}^{n}\left(\begin{array}{l}
n \\
k
\end{array}\right)(1-c)^{k} c^{n-k} \log _{a}\left(x_{k}\right) \\
& \leq 2+\log _{a}\left[\sum_{k=0}^{n}\left(\begin{array}{l}
n \\
k
\end{array}\right)(1-c)^{k} c^{n-k} x_{k}\right] \\
& =2+\log _{a}\left[\sum_{k=1}^{n-1}\left(\begin{array}{l}
n \\
k
\end{array}\right)(1-c)^{k} c^{n-k} k+c^{n}+(1-c)^{n}\right] \\
& \leq 2+\log _{a}\left[\sum_{k=1}^{n-1}\left(\begin{array}{l}
n \\
k
\end{array}\right)(1-c)^{k} c^{n-k} k+n(1-c)^{n}\right] \\
& =2+\log _{a}\left[\sum_{k=0}^{n}\left(\begin{array}{l}
n \\
k
\end{array}\right)(1-c)^{k} c^{n-k} k\right] \\
& =2+\log _{a}[(1-c) n] \\
& =1+\log _{a} n
\end{aligned}
$$

The last inequality holds since for any $c \leq \frac{1}{2}$ and any $n \geq 2$ it holds that $c^{n}+(1-c)^{n} \leq 2(1-c)^{n} \leq n(1-c)^{n}$.

A final immediate consequence of Lemmas 8.3.1 - 8.3.3 is the following theorem.

Theorem 8.3.4. For any $c \leq \frac{1}{2}$ and any $n \geq 2, e_{c}(n) \leq e^{\frac{1-c}{c^{2}}}\left(\log _{\frac{1}{1-c}} n+1\right)$.

Remark: Since $e_{c}(n)>e_{\bar{c}}(n)$ when $c<\bar{c}$, the upper bound for $c=\frac{1}{2}$ is also valid for any $c>\frac{1}{2}$.

We showed that the expected number of rounds of the $c$-bisection auction is bounded from above by a function that is logarithmic in the number of players. A comparison of the bound with the computed results suggests that this bound is not tight. Computer results also show that for a fixed value of $c$ the ratio between the bound and the computed result is approximately constant (as a function of $n$ ), implying that the bound is likely to have the correct order of magnitude.

\subsubsection{The expected number of queries}

Let $b_{c}(k)$ be the expected number of queries of the auction with $k$ active players, given that the decision of the active player with the lowest ranking is yes in the 
current round; $b_{c}^{*}(k)$ be the expected number of queries given that this decision is no. Notice that in a round with $k$ active players $k$ queries are performed. Following the same argumentation as we used for determining the formula for the expected number of rounds we find that for any $n \geq 2$

$$
b_{c}(n)=n+\sum_{k=1}^{n-1}\left(\begin{array}{c}
n-1 \\
k
\end{array}\right)(1-c)^{k} c^{n-1-k}\left[(1-c) b_{c}(k+1)+c b_{c}^{*}(k+1)\right] .
$$

Again, notice that when player $i_{r+1}=i_{r}$ says no player $j_{r+1}$ says yes with certainty, which causes player $i_{r+1}$ to drop out of the auction. Thus, $b_{c}^{*}(2)=2$ and for all $k>1$ it holds that $b_{c}^{*}(k+1)=1+b_{c}(k)$. This is used in Appendix 8.6 to derive the following recursive relation. For any $n \geq 2$

$$
\left[1-(1-c)^{n}\right] b_{c}(n)=n+(n-1)(1-c) c^{n-1}+c-c^{n}+\sum_{k=2}^{n-1}\left(\begin{array}{l}
n \\
k
\end{array}\right)(1-c)^{k} c^{n-k} b_{c}(k) \text {. }
$$

Now notice that since in the first round player $i_{1}$ says yes with certainty, the expected number of queries in the auction of $n$ players is equal to $b_{c}(n)$. Thus using formula 8.4 we can compute the expected number of queries performed in the auction of $n$ players. Plugging in $n=2$ yields $b_{c}(2)=\frac{2+2 c(1-c)}{c(2-c)}$. All other values can be determined recursively. Table 8.2 in Appendix 8.6 presents the computational results for different values of $c$ in the auction with up to 100 players (data is within an accuracy of 0.1). Figure 8.2(a) demonstrates that for a fixed value of $c$ the expected number of queries increases in the number of players participating in the auction. Figure 8.2(b) shows that for a fixed number of players the expected number of queries decreases as $c$ becomes larger.

Generally we show that the expected number of queries is bounded from above by a function that is linear in the number of players. To prove this we introduce several notations and lemmas.

Define $B_{2}=\frac{2+2 c(1-c)}{c(2-c)}$ and

$$
B_{n}=n+(n-1)(1-c) c^{n-1}+c-c^{n}+\sum_{k=2}^{n-1}\left(\begin{array}{l}
n \\
k
\end{array}\right)(1-c)^{k} c^{n-k} B_{k}
$$

for any $n>2$.

Recall that $D_{n}=\prod_{k=1}^{n} \frac{1}{1-(1-c)^{k}}$. 


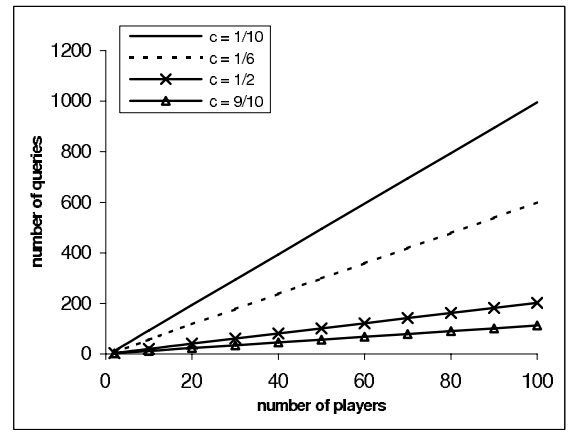

a)

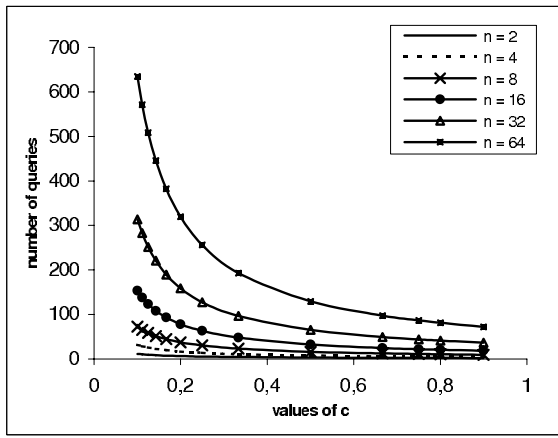

b)

Figure 8.2: The expected number of queries (a) for different fixed values of $c$; (b) for different fixed numbers of players.

Lemma 8.3.5. For any $n \geq 2, b_{c}(n) \leq B_{n} \cdot D_{n}$.

Proof. The proof is identical to the proof of Lemma 8.3.1 if we replace $e_{c}(k)$ by $b_{c}(k)$ and $E_{k}$ by $B_{k}$ for all $2 \leq k \leq n$.

From Lemma 8.3.2 we know that for any $n \geq 2, D_{n} \leq e^{\frac{1-c}{c^{2}}}$. Now we find a bound on $B_{n}$.

Lemma 8.3.6. For any $n \geq 2, B_{n} \leq\left(\frac{2}{c}+\frac{1}{2}\right)(n+1)$.

Proof. The proof is by induction on $n$. The basis of the induction holds since it can be easily shown that $B_{2}<3\left(\frac{2}{c}+\frac{1}{2}\right)$. Now suppose that $B_{k} \leq\left(\frac{2}{c}+\frac{1}{2}\right)(k+1)$ for any $2 \leq k \leq n-1$. Using the induction hypothesis,

$$
\begin{aligned}
B_{n} & =n+(n-1)(1-c) c^{n-1}+c-c^{n}+\sum_{k=2}^{n-1}\left(\begin{array}{l}
n \\
k
\end{array}\right)(1-c)^{k} c^{n-k} B_{k} \\
& \leq n+(n-1)(1-c) c^{n-1}+c-c^{n}+\sum_{k=2}^{n-1}\left(\begin{array}{l}
n \\
k
\end{array}\right)(1-c)^{k} c^{n-k}\left(\frac{2}{c}+\frac{1}{2}\right)(k+1) \\
& \leq 2 n+c+\left(\frac{2}{c}+\frac{1}{2}\right) \sum_{k=0}^{n}\left(\begin{array}{l}
n \\
k
\end{array}\right)(1-c)^{k} c^{n-k} k+\left(\frac{2}{c}+\frac{1}{2}\right) \sum_{k=0}^{n}\left(\begin{array}{l}
n \\
k
\end{array}\right)(1-c)^{k} c^{n-k} \\
& =2 n+c+\left(\frac{2}{c}+\frac{1}{2}\right)(1-c) n+\left(\frac{2}{c}+\frac{1}{2}\right)
\end{aligned}
$$




$$
\begin{aligned}
& =\left(\frac{2}{c}+\frac{1}{2}\right)(n+1)+c\left(1-\frac{n}{2}\right) \\
& \leq\left(\frac{2}{c}+\frac{1}{2}\right)(n+1) .
\end{aligned}
$$

The last inequality holds since $n \geq 2$.

A final immediate consequence of Lemmas 8.3.2, 8.3.5 and 8.3.6 is the following theorem.

Theorem 8.3.7. For any integer $n \geq 2, b_{c}(n) \leq e^{\frac{1-c}{c^{2}}}\left(\frac{2}{c}+\frac{1}{2}\right)(n+1)$.

We showed that the expected number of queries is bounded from above by a function that is linear in the number of players. Again, a comparison of the bound with the computed results suggests that this bound is not tight. Computer results also show that for a fixed value of $c$ the ratio between the bound and the computed result is approximately constant (as a function of $n$ ), implying that the bound is likely to have the correct order of magnitude.

\subsection{Efficiency of the $c$-bisection auction}

In this section we investigate the efficiency of the $c$-bisection auction when the bluff equilibrium is played. In particular we compute the probability of inefficient allocation and the expected loss of welfare. Here for simplicity of argumentation we focus on the setting where valuations of players are independently drawn from the uniform distribution in $[0,1)$.

In order to compute these measures of inefficiency it is convenient to consider the direct revelation mechanism associated with the bluff equilibrium. We construct a direct auction that mimics the bluff strategies of the $c$-bisection auction.

\subsubsection{The direct $c$-bisection auction}

Consider the following direct auction $\left(w_{d}, p_{d}\right)$, called the direct $c$-bisection auction . For $r \in \mathbb{N}$, write $I_{r}:=\left[1-(1-c)^{r-1}, 1-(1-c)^{r}\right)$. ${ }^{3}$ Note that the intervals $I_{1}, I_{2}, \ldots$ partition the unit interval $[0,1)$ from which valuations are drawn. Now let $v=\left(v_{i}\right)_{i \in N}$ be a profile of valuations. Write $I_{r}(v):=I_{r} \cap\left\{v_{i} \mid i \in N\right\}$ - the set of

\footnotetext{
${ }^{3}$ In case of a general density function $F(v)$ in $[\alpha, \beta)$ intervals $I_{r}$ are defined recursively as follows. Write $I_{r}=\left[\alpha_{r}, \beta_{r}\right)$ where $\alpha_{1}=\alpha, \alpha_{r}=\beta_{r-1}$ and $\beta_{r}$ is chosen such that $\frac{F\left(\beta_{r}\right)-F\left(\alpha_{r}\right)}{F(\beta)-F\left(\alpha_{r}\right)}=c$.
} 
valuations that belong to the interval $I_{r}$. Let $r(v)$ be the highest natural number $r$ for which $I_{r}(v)$ is not empty. Among players whose valuations belong to the interval $I_{r(v)}$ the one with the lowest ranking is declared to be the winner. So the winner $w_{d}$ is defined by

$$
w_{d}(v):=\min \left\{i \in N \mid v_{i} \in I_{r(v)}\right\} .
$$

Let $s(v)$ be the highest natural number $r$ for which $I_{r} \cap\left\{v_{i} \mid i \in N \backslash\left\{w_{d}(v)\right\}\right\}$ is not empty. The price the winner pays is equal to the lower bound of interval $I_{s(v)}$ if all players whose valuations belong to this interval have a ranking higher than the winner. Otherwise the price equals the upper bound of this interval. So the payment $p_{d}$ is defined by

$$
p_{d}(v):= \begin{cases}1-(1-c)^{s(v)-1} & \text { if } i>w_{d}(v) \text { for all } i \in I_{s(v)}(v) \\ 1-(1-c)^{s(v)} & \text { else. }\end{cases}
$$

Notice that the first condition always holds if $\left|I_{r(v)}\right|>1$, i.e. if $s(v)=r(v)$. If $I_{r(v)}$ contains only one valuation, the payment depends on the ranking of the players with valuations in $I_{s(v)}$.

Example. Consider the same example as in Section 2.2 with players $A \prec B \prec$ $C \prec D \prec E$ whose valuations are $0.43,0.71,0.38,0.79$ and 0.86 , respectively. Suppose that in the direct $c$-bisection auction with $c=1 / 2$ the players truthfully report their valuations. Then $r(v)=s(v)=3$. Players with valuation in $I_{3}$ are players $\mathrm{D}$ and E. Player $D$ has ranking lower than player $E$ so he is the winner. The price he pays for the object is equal to the lower bound of $I_{3}$, namely 0.75 . So we get the same outcome as the one we found in Section 2.2.

We will now show that $\left(w_{d}, p_{d}\right)$ equals the direct auction $(w \circ b, p \circ b)$ where $w$ and $p$ are defined as in Section 7.2 and $b=\left(b_{i}\right)_{i \in N}$ is the bluff equilibrium.

Theorem 8.4.1. For any realization $v=\left(v_{i}\right)_{i \in N}$ of valuations it holds that $w_{d}(v)=$ $(w \circ b)(v)$ and $p_{d}(v)=(p \circ b)(v)$. Consequently, truthful bidding is a dominant strategy in the direct auction $\left(w_{d}, p_{d}\right)$.

Proof. Let $v=\left(v_{i}\right)_{i \in N}$ be a realization of valuations. By Theorem 7.4.2 we know that the price will always increase. Consider the round $s(v)$ in which the price is equal to $1-(1-c)^{s(v)-1}$ and the query price is equal to $1-(1-c)^{s(v)}$. The active bidders in round $s(v)$ are $(w \circ b)(v)$, all bidders $i$ with $v_{i} \in I_{s(v)}$, and -possibly-one more bidder $i^{*}$ with $v_{i^{*}} \in I_{s(v)-1}$ who happened to be the bidder with the lowest ranking number among those bidders that were active in the previous round and said yes in that round. We distinguish three cases. 


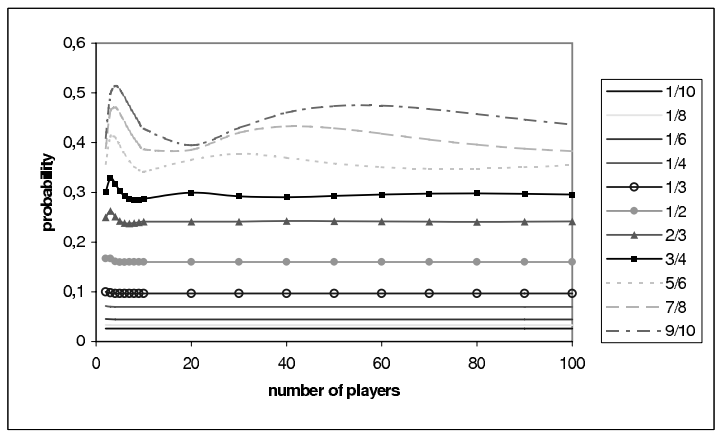

Figure 8.3: The probability of inefficient allocation.

Case 1. If $i^{*}$ exists. Bidder $i^{*}$ will say no in this round $s(v)$, and the next active bidder, say $j$, in the bidding order will say yes. If $j=(w \circ b)(v)$ then all other active bidders say no. So, $(w \circ b)(v)=\min \left\{i \in N \mid v_{i} \in I_{r(v)}\right\}$ and $(p \circ b)(v)=1-(1-c)^{s(v)-1}$. If $j \neq(w \circ b)(v)$ then both $j$ and $(w \circ b)(v)$ say yes in this round, while all other active bidders say no. In the next round though $j$ will say $n o$ and $(w \circ b)(v)$ says yes. Hence in this case $(w \circ b)(v)=\min \left\{i \in N \mid v_{i} \in I_{r(v)}\right\}$ and $(p \circ b)(v)=1-(1-c)^{s(v)}$.

Case 2. If $i^{*}$ does not exist and $i>(w \circ b)(v)$ for all $i \in I_{s(v)}(v)$. In this case $(w \circ b)(v)$ says yes in round $s(v)$ while all other active bidders say no. Hence $(w \circ b)(v)=\min \left\{i \in N \mid v_{i} \in I_{r(v)}\right\}$ and $(p \circ b)(v)=1-(1-c)^{s(v)-1}$.

Case 3. When not in Case 1 or 2. Then an active bidder $j \neq(w \circ b)(v)$ says yes in round $s(v)$ together with $(w \circ b)(v)$, while all other bidders say no. In round $s(v)+1$ bidder $j$ says no and bidder $(w \circ b)(v)$ says yes. Hence in this case $(w \circ b)(v)=\min \left\{i \in N \mid v_{i} \in I_{r(v)}\right\}$ and $(p \circ b)(v)=1-(1-c)^{s(v)}$.

So we have shown that for any realization of valuations $v=\left(v_{i}\right)_{i \in N}$ the outcome $\left(w_{d}(v), p_{d}(v)\right)$ equals the outcome of the $c$-bisection auction when players, having these valuations, follow the bluff strategies. Consequently, by the revelation principle (see e.g. Mas-Colell et al. [41]) truth telling is a dominant strategy in the direct $c$-bisection auction. Due to this result the efficiency performance of both the $c$ bisection query auction under the bluff equilibrium and the direct $c$-bisection auction under the truth telling equilibrium are the same. Thus it suffices to compute the probability of inefficient allocation and the expected loss of welfare for the direct $c$-bisection auction under the truth telling equilibrium. 


\subsubsection{The probability of inefficient allocation}

We derive a recursive formula for the probability of inefficient allocation and give an upper bound for the function defined by this formula. First notice that the direct $c$-bisection auction restricted to the interval $[c, 1)$ with $k$ players having valuations uniformly drawn from this interval has identical form and structure as the original direct auction with $k$ players having valuations uniformly drawn from $[0,1)$.

Let us denote by $P_{c}(n)$ the probability that the auction with $n$ players terminates in an inefficient allocation. First, consider the case where the valuations of all players are smaller than $c$. The probability of this event is $c^{n}$. In this case the auction is only efficient if the player with the lowest ranking has the highest valuation. By symmetry this happens with probability $\frac{1}{n}$. Thus this case contributes $\frac{n-1}{n} c^{n}$ to $P_{c}(n)$. Next consider the case where $k$ players have valuations larger than or equal to $c$ and $n-k$ players have valuations smaller than $c$. It happens with probability $\left(\begin{array}{l}n \\ k\end{array}\right) c^{n-k}(1-c)^{k}$. For $k=1$ the auction is efficient, so this case adds zero to $P_{c}(n)$. For $k>1$ the auction can be inefficient and due to the structural similarity, inefficiency takes place with probability $P_{c}(k)$. Hence,

$$
P_{c}(n)=\frac{n-1}{n} c^{n}+\sum_{k=2}^{n}\left(\begin{array}{l}
n \\
k
\end{array}\right) c^{n-k}(1-c)^{k} P_{c}(k) .
$$

This can be rewritten to the following recursive relation, $P_{c}(2)=\frac{1}{2} \cdot \frac{c}{2-c}$ and for $n \geq 3$

$$
\left[1-(1-c)^{n}\right] P_{c}(n)=\frac{n-1}{n} c^{n}+\sum_{k=2}^{n-1}\left(\begin{array}{l}
n \\
k
\end{array}\right) c^{n-k}(1-c)^{k} P_{c}(k)
$$

Direct computation of this expression for different combinations of $n$ and $c$ gives the values that are plotted in Figure 8.3.

We will show that the probability of inefficiency $P_{c}(n)$ is smaller than $c$, and also bounded away from zero.

Theorem 8.4.2. For all $n \geq 2, P_{c}(n) \leq c$ and $P_{c}(n) \geq \frac{c}{2(2-c)} \cdot e^{-Z_{c}}$ where

$$
Z_{c}:=\sum_{k=3}^{\infty} \frac{c^{k}+k \lambda c^{k-1}}{1-\lambda^{k}-k \lambda c^{k-1}-c^{k}} \text { with } \lambda=1-c .
$$

Proof. First we show that $P_{c}(n) \leq c$. The proof is by induction on $n$. The basis of induction holds since $P_{c}(2)=\frac{c}{2(2-c)} \leq c$. Suppose that $P_{c}(k) \leq c$ for all $2 \leq k \leq$ $n-1$. Then 


$$
\begin{aligned}
P_{c}(n) & =\frac{1}{1-(1-c)^{n}}\left[\frac{n-1}{n} \cdot c^{n}+\sum_{k=2}^{n-1}\left(\begin{array}{l}
n \\
k
\end{array}\right)(1-c)^{k} c^{n-k} \cdot P_{c}(k)\right] \\
& \leq \frac{1}{1-(1-c)^{n}}\left[c^{n}+\sum_{k=2}^{n-1}\left(\begin{array}{l}
n \\
k
\end{array}\right)(1-c)^{k} c^{n-k} \cdot c\right] \\
& =\frac{1}{1-(1-c)^{n}}\left[c^{n}+c\left(1-c^{n}-n(1-c) c^{n-1}-(1-c)^{n}\right)\right] \\
& =\frac{c\left(1-(1-c)^{n}\right)}{1-(1-c)^{n}}+\frac{c^{n}-c^{n+1}-n(1-c) c^{n}}{1-(1-c)^{n}} \\
& =c+\frac{c^{n}(1-c)(1-n)}{1-(1-c)^{n}} \\
& \leq c .
\end{aligned}
$$

The first inequality holds by the induction assumption and the fact that $\frac{n-1}{n}<1$. The last inequality holds since $n \geq 2$. This concludes the proof for the upper bound on $P_{c}(n)$.

Now we show that $P_{c}(n) \geq \frac{c}{2(2-c)} \cdot e^{-Z_{c}}$. Define $B_{c}(n)$ as follows. Let $B_{c}(2):=$ $\frac{c}{2(2-c)}$ and for $n \geq 3$

$$
B_{c}(n):=\frac{1}{1-\lambda^{n}}\left[\sum_{k=2}^{n-1}\left(\begin{array}{l}
n \\
k
\end{array}\right) \lambda^{k} c^{n-k} B_{c}(k)\right]
$$

A simple induction argument shows that $B_{c}(n) \leq P_{c}(n)$ for all $n$. We will show that $B_{c}(n) \geq \frac{c}{2(2-c)} \cdot e^{-Z_{c}}$ for all $n$. Define $Q_{2}(c):=1$ and for $n \geq 3$

$$
Q_{c}(n):=\frac{1-\lambda^{n}-n \lambda c^{n-1}-c^{n}}{1-\lambda^{n}} \cdot Q_{c}(n-1)=\prod_{k=3}^{n} \frac{1-\lambda^{k}-k \lambda c^{k-1}-c^{k}}{1-\lambda^{k}}
$$

We will first show that for all $n \geq 2, B_{c}(k) \geq B_{c}(2) \cdot Q_{c}(n)$ holds for all $2 \leq k \leq n$. Clearly this holds for $n=2$. Take $n \geq 3$. Assume that for all $2 \leq k \leq n-1$ we have that $B_{c}(k) \geq B_{c}(2) \cdot Q_{c}(n-1)$. Since $0<Q_{c}(n) \leq Q_{c}(n-1)$ we have that 
$B_{c}(k) \geq B_{c}(2) \cdot Q_{c}(n)$ for all $2 \leq k \leq n-1$. For $k=n$,

$$
\begin{aligned}
B_{c}(n) & =\frac{1}{1-\lambda^{n}}\left[\sum_{k=2}^{n-1}\left(\begin{array}{l}
n \\
k
\end{array}\right) \lambda^{k} c^{n-k} B_{c}(k)\right] \\
& \geq \frac{1}{1-\lambda^{n}}\left[\sum_{k=2}^{n-1}\left(\begin{array}{l}
n \\
k
\end{array}\right) \lambda^{k} c^{n-k} Q_{c}(n-1) B_{c}(2)\right] \\
& =\frac{1}{1-\lambda^{n}} \cdot Q_{c}(n-1) \cdot B_{c}(2) \cdot\left[\sum_{k=2}^{n-1}\left(\begin{array}{l}
n \\
k
\end{array}\right) \lambda^{k} c^{n-k}\right] \\
& =\frac{1-\lambda^{n}-n \lambda c^{n-1}-c^{n}}{1-\lambda^{n}} \cdot Q_{c}(n-1) \cdot B_{c}(2)=B_{c}(2) \cdot Q_{c}(n)
\end{aligned}
$$

which shows that $B_{c}(n) \geq B_{c}(2) \cdot Q_{c}(n)$. Now notice that $Z_{c}>0$. So, $Q_{c}(2)=1 \geq$ $e^{-Z_{c}}$, while for $n \geq 3$

$$
\begin{aligned}
\log Q_{c}(n) & =\sum_{k=3}^{n}\left[\log \left(1-\lambda^{k}-k \lambda c^{k-1}-c^{k}\right)-\log \left(1-\lambda^{k}\right)\right] \\
& =-\sum_{k=3}^{n}\left[\log \left(1-\lambda^{k}\right)-\log \left(1-\lambda^{k}-k \lambda c^{k-1}-c^{k}\right)\right] \\
& \geq-\sum_{k=3}^{n} \frac{k \lambda c^{k-1}+c^{k}}{1-\lambda^{k}-k \lambda c^{k-1}-c^{k}} \geq-Z_{c},
\end{aligned}
$$

where the first inequality follows from the fact that $\log y-\log x \leq \frac{y-x}{x}$ for $y>x$. Hence, since $B_{c}(2):=\frac{c}{2(2-c)}, P_{c}(n) \geq B_{c}(n) \geq B_{c}(2) \cdot Q_{c}(n) \geq \frac{c}{2(2-c)} \cdot e^{-Z_{c}}$ for all $n \geq 2$.

This theorem shows in particular that by choosing an appropriate fraction $c$ in the auction we can make the probability of inefficiency as small as we like, independent of the number of players!

\subsubsection{The expected loss of welfare}

The welfare of an auction is equal to the valuation of the winner. Thus given a realization of valuations $v=\left(v_{i}\right)_{i \in N}$, the welfare achieved by the auction is the valuation of $w_{d}(v):=\min \left\{i \mid v_{i} \in I_{r(v)}\right\}$. The maximum welfare, given $v$, is $\max \left\{v_{i} \mid i \in N\right\}=\max \left\{v_{i} \mid v_{i} \in I_{r(v)}\right\}$. Thus, the loss $L(v)$ of welfare is

$$
L(v)=\max \left\{v_{i} \mid v_{i} \in I_{r(v)}\right\}-v_{w_{d}(v)} .
$$

The expected loss of welfare, denoted by $L_{c}(n)$, is the expected value of this difference. To estimate the value of $L_{c}(n)$ we simulated the direct $c$-bisection auction and 


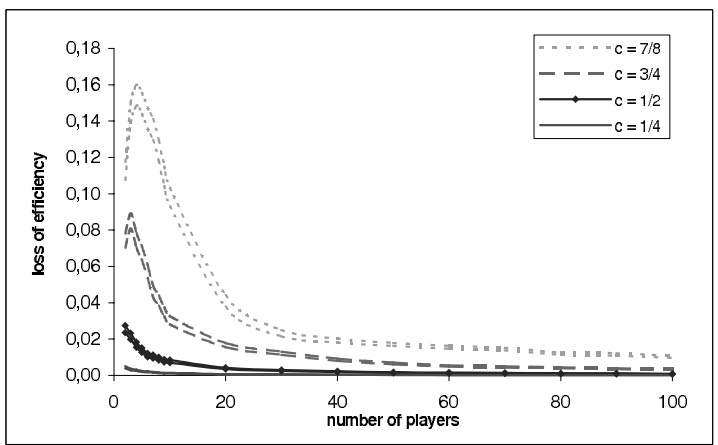

Figure 8.4: The expected loss of welfare, $99 \%$ confidence interval.

ran it for valuations uniformly and independently drawn from the interval $[0,1)$. For each combination of value $c$ and number of players $n$ we ran 10,000 trials. Figure 8.4 shows the $99 \%$ confidence interval for the expected loss of welfare. It is interesting to notice that the maximum expected loss does not arrive at the minimum number of players.

In general, we show the following statement. ${ }^{4}$

Theorem 8.4.3. For all $n \geq 2, L_{c}(n) \leq c^{2}$.

Proof. Let $v=\left(v_{i}\right)_{i \in N}$ be a realization of valuations for which the allocation in the direct $c$-bisection auction is not efficient. In other words, $\max \left\{v_{i} \mid v_{i} \in I_{r(v)}\right\}>$ $v_{w_{d}(v)}$. Since the valuation of $w_{d}(v)$ is an element of $I_{r(v)}$ we get that

$$
L(v) \leq \operatorname{length}\left(I_{r(v)}\right) \leq c .
$$

Hence, $L_{c}(n) \leq c \cdot P_{c}(n)$. Applying the first result of Theorem 8.4 .2 completes the proof.

As for probability of inefficient allocation, by choosing an appropriate fraction $c$ in the auction we can limit the expected loss of welfare to an arbitrary chosen level, independent of the number of players. The following result shows that the expected loss of welfare even tends to zero when the number of participants becomes large.

\footnotetext{
${ }^{4}$ This result can not be generalized to an arbitrary density function $F(v)$ since it is based on the lengths of intervals $I_{r}$ which entirely depend on distribution of valuations.
} 
Theorem 8.4.4. The expected loss of welfare $L_{c}(n)$ tends to zero for large $n$.

Proof. Let $v=\left(v_{i}\right)_{i \in N}$ be a realization of valuations. Since the valuation of $w_{d}(v)$ is an element of $I_{r(v)}$ we get that

$$
L(v)=\max \left\{v_{i} \mid v_{i} \in I_{r(v)}\right\}-v_{w_{d}(v)} \leq \operatorname{length}\left(I_{r(v)}\right)=c \cdot(1-c)^{r(v)-1} .
$$

Thus, conditioning in the direct $c$-bisection auction on the event that exactly $k$ bidders have a valuation in $I_{r(v)}$ and consequently the $n-k$ remaining bidders have a valuation lower than $1-(1-c)^{r(v)-1}$, it follows that

$$
\begin{aligned}
L_{c}(n) & \leq \frac{n-1}{n} c \cdot c^{n}+\sum_{r=2}^{\infty} \sum_{k=2}^{n}\left(\begin{array}{l}
n \\
k
\end{array}\right)\left[c(1-c)^{r-1}\right]^{k}\left[1-(1-c)^{r-1}\right]^{n-k} \cdot c(1-c)^{r-1} \\
& \leq c^{n+1}+\sum_{r=2}^{\infty} c(1-c)^{r-1} \sum_{k=0}^{n}\left(\begin{array}{l}
n \\
k
\end{array}\right)\left[c(1-c)^{r-1}\right]^{k}\left[1-(1-c)^{r-1}\right]^{n-k} \\
& =c^{n+1}+\sum_{r=2}^{\infty} c(1-c)^{r-1}\left[c(1-c)^{r-1}+1-(1-c)^{r-1}\right]^{n} \\
& =c^{n+1}+\sum_{r=2}^{\infty} c(1-c)^{r-1}\left[1-(1-c)^{r}\right]^{n} .
\end{aligned}
$$

We will show that

$$
S_{c}(n):=\sum_{r=2}^{\infty} c(1-c)^{r-1}\left[1-(1-c)^{r}\right]^{n}
$$

tends to zero for large $n$. Take an $\varepsilon>0$. Take $r^{*}$ such that

$$
\sum_{r=r^{*}}^{\infty} c(1-c)^{r-1}<\frac{1}{2} \varepsilon .
$$

Take $N$ such that for all $n>N$

$$
\sum_{r=2}^{r^{*}-1} c(1-c)^{r-1}\left[1-(1-c)^{r^{*}}\right]^{n}<\frac{1}{2} \varepsilon .
$$

Then for all $n>N$,

$$
\begin{aligned}
S_{c}(n) & =\sum_{r=2}^{r^{*}-1} c(1-c)^{r-1}\left[1-(1-c)^{r}\right]^{n}+\sum_{r=r^{*}}^{\infty} c(1-c)^{r-1}\left[1-(1-c)^{r}\right]^{n} \\
& \leq \sum_{r=2}^{r^{*}-1} c(1-c)^{r-1}\left[1-(1-c)^{r^{*}}\right]^{n}+\sum_{r=r^{*}}^{\infty} c(1-c)^{r-1} \\
& <\frac{1}{2} \varepsilon+\frac{1}{2} \varepsilon=\varepsilon
\end{aligned}
$$

which shows our claim. 


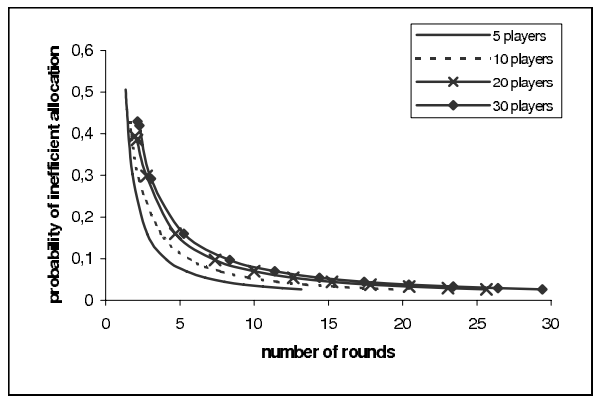

a)

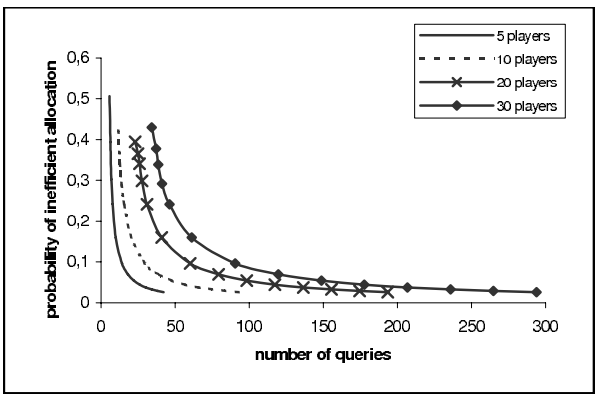

b)

Figure 8.5: The trade-off between (a) the probability of inefficient allocation and the number of rounds; (b) the probability of inefficient allocation and the number of queries.

\subsection{Concluding remarks: trade-off between efficiency and running time}

From the analysis above we derive the following relation between the value of $c$, the level of efficiency and the running time. For a fixed number of players, a smaller fraction $c$ leads to a lower expected loss of welfare and lower probability of inefficient allocation. But at the same time it leads to a higher expected number of rounds and queries. Thus, increasing running time is a price that we have to pay for increasing efficiency. Depending on the priorities of the auctioneer he may trade off efficiency against running time. Figure 8.5 shows, for some fixed $n$, the relation between the expected running time and the probability of inefficient allocation. These relations are built on computational results based on recursive formulas 8.2, 8.4 and 8.5. Figure 8.6 shows, for some fixed $n$, the relation between the expected running time and the expected loss of welfare. Because we do not have exact values for the expected loss of welfare we estimated the values by taking the middle point of the $99 \%$ confidence interval from the simulation results reported above. Notice that in Figure 8.6(a) the trade-off curves drawn for different numbers of players almost coincide with each other. It means that in order to get the desired level of efficiency we need to run the auction that in expectation takes the same number of rounds for any number of players participating in the auction (of course, the choice of $c$ to be used in this auction will depend on the number of players). This explains why for 


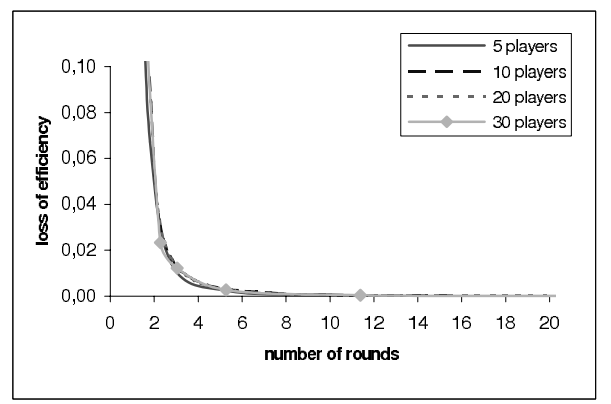

a)

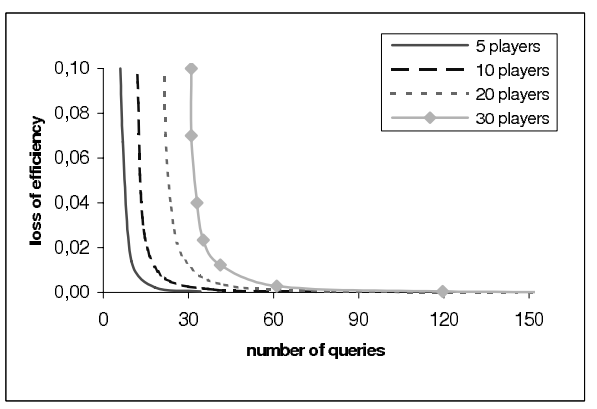

b)

Figure 8.6: The trade-off between (a) the loss of welfare and the number of rounds; (b) the loss of welfare and the number of queries.

the same level of efficiency more players require more queries to be asked, which is demonstrated in Figure 8.6(b). 


\subsection{Appendix}

\section{Derivation of formula 8.2}

Let us denote by $P_{k}^{n}=\left(\begin{array}{l}n \\ k\end{array}\right)(1-c)^{k} c^{n-k}$. Using the facts that $e_{c}^{*}(2)=1$ and $e_{c}^{*}(k+1)=e_{c}(k)$ for all $k \geq 2$ we can rewrite formula 8.1 as follows:

$$
\begin{aligned}
e_{c}(n)= & 1+\sum_{k=1}^{n-1} P_{k}^{n-1}\left[(1-c) e_{c}(k+1)+c e_{c}^{*}(k+1)\right] \\
= & 1+(1-c) \sum_{k=1}^{n-2} P_{k}^{n-1} e_{c}(k+1)+(1-c) P_{n-1}^{n-1} e_{c}(n)+c \sum_{k=2}^{n-1} P_{k}^{n-1} e_{c}^{*}(k+1)+ \\
& c P_{1}^{n-1} e_{c}^{*}(2) \\
= & 1+(1-c) \sum_{k=1}^{n-2} P_{k}^{n-1} e_{c}(k+1)+(1-c)^{n} e_{c}(n)+c \sum_{k=2}^{n-1} P_{k}^{n-1} e_{c}(k)+ \\
& (n-1)(1-c) c^{n-1} \\
= & 1+(1-c)^{n} e_{c}(n)+(n-1)(1-c) c^{n-1}+(1-c) \sum_{k=2}^{n-1} P_{k-1}^{n-1} e_{c}(k)+ \\
& c \sum_{k=2}^{n-1} P_{k}^{n-1} e_{c}(k) \\
= & 1+(1-c)^{n} e_{c}(n)+(n-1)(1-c) c^{n-1}+\sum_{k=2}^{n-1}\left[(1-c) P_{k-1}^{n-1}+c P_{k}^{n-1}\right] e_{c}(k) \\
= & 1+(1-c)^{n} e_{c}(n)+(n-1)(1-c) c^{n-1}+\sum_{k=2}^{n-1} P_{k}^{n} e_{c}(k) .
\end{aligned}
$$

This can be rewritten to

$$
\left[1-(1-c)^{n}\right] e_{c}(n)=1+(n-1)(1-c) c^{n-1}+\sum_{k=2}^{n-1}\left(\begin{array}{l}
n \\
k
\end{array}\right)(1-c)^{k} c^{n-k} e_{c}(k) .
$$




\section{Derivation of formula 8.4}

Recall that $P_{k}^{n}=\left(\begin{array}{l}n \\ k\end{array}\right)(1-c)^{k} c^{n-k}$ Using the facts that $b^{*}(2)=2$ and $b^{*}(k+1)=$ $b(k)+1$ for all $k \geq 2$, we get from formula 8.3 that

$$
\begin{aligned}
b_{c}(n)= & n+\sum_{k=1}^{n-1} P_{k}^{n-1}\left[(1-c) b_{c}(k+1)+c b_{c}^{*}(k+1)\right] \\
= & n+(1-c) \sum_{k=1}^{n-2} P_{k}^{n-1} b_{c}(k+1)+(1-c) P_{n-1}^{n-1} b_{c}(n)+c \sum_{k=2}^{n-1} P_{k}^{n-1} b_{c}^{*}(k+1)+ \\
& c P_{1}^{n-1} b_{c}^{*}(2) \\
= & n+(1-c) \sum_{k=1}^{n-2} P_{k}^{n-1} b_{c}(k+1)+(1-c)^{n} b_{c}(n)+c \sum_{k=2}^{n-1} P_{k}^{n-1}\left[b_{c}(k)+1\right]+ \\
& 2(n-1)(1-c) c^{n-1} \\
= & n+(1-c)^{n} b_{c}(n)+2(n-1)(1-c) c^{n-1}+(1-c) \sum_{k=2}^{n-1} P_{k-1}^{n-1} b_{c}(k)+ \\
& c \sum_{k=2}^{n-1} P_{k}^{n-1} b_{c}(k)+c \sum_{k=2}^{n-1} P_{k}^{n-1} \\
& n+(1-c)^{n} b_{c}(n)+2(n-1)(1-c) c^{n-1}+c-c^{n}-(n-1)(1-c) c^{n-1}+ \\
& \sum_{k=2}^{n-1}\left[(1-c) P_{k-1}^{n-1}+c P_{k}^{n-1}\right] b_{c}(k) \\
= & n+(1-c)^{n} b_{c}(n)+(n-1)(1-c) c^{n-1}+c-c^{n}+\sum_{k=2}^{n-1} P_{k}^{n} b_{c}(k) .
\end{aligned}
$$

Rewriting yields, for any $n \geq 2$,

$$
\left[1-(1-c)^{n}\right] b_{c}(n)=n+(n-1)(1-c) c^{n-1}+c-c^{n}+\sum_{k=2}^{n-1}\left(\begin{array}{l}
n \\
k
\end{array}\right)(1-c)^{k} c^{n-k} b_{c}(k)
$$


Table 8.1: The expected number of rounds $e_{c}(n)$ in the $c$-bisection auction.

\begin{tabular}{r|rrrrrrrrrrr}
$n \backslash c$ & $1 / 10$ & $1 / 8$ & $1 / 6$ & $1 / 4$ & $1 / 3$ & $1 / 2$ & $2 / 3$ & $3 / 4$ & $5 / 6$ & $7 / 8$ & $9 / 10$ \\
\hline 2 & 5.737 & 4.733 & 3.727 & 2.714 & 2.200 & 1.667 & 1.375 & 1.267 & 1.171 & 1.127 & 1.101 \\
3 & 8.901 & 7.230 & 5.555 & 3.873 & 3.021 & 2.143 & 1.663 & 1.483 & 1.319 & 1.240 & 1.193 \\
4 & 11.273 & 9.102 & 6.927 & 4.742 & 3.638 & 2.505 & 1.891 & 1.660 & 1.446 & 1.341 & 1.277 \\
5 & 13.172 & 10.600 & 8.024 & 5.437 & 4.131 & 2.794 & 2.076 & 1.807 & 1.557 & 1.431 & 1.353 \\
6 & 14.753 & 11.848 & 8.938 & 6.016 & 4.542 & 3.035 & 2.230 & 1.931 & 1.654 & 1.512 & 1.423 \\
7 & 16.109 & 12.918 & 9.721 & 6.513 & 4.895 & 3.241 & 2.361 & 2.037 & 1.738 & 1.584 & 1.486 \\
8 & 17.296 & 13.854 & 10.407 & 6.947 & 5.203 & 3.421 & 2.475 & 2.129 & 1.813 & 1.650 & 1.545 \\
9 & 18.350 & 14.686 & 11.016 & 7.334 & 5.477 & 3.581 & 2.576 & 2.211 & 1.879 & 1.709 & 1.598 \\
10 & 19.299 & 15.435 & 11.565 & 7.681 & 5.724 & 3.726 & 2.667 & 2.283 & 1.939 & 1.762 & 1.647 \\
20 & 25.647 & 20.443 & 15.233 & 10.006 & 7.373 & 4.690 & 3.275 & 2.760 & 2.312 & 2.102 & 1.971 \\
30 & 29.417 & 23.418 & 17.412 & 11.387 & 8.353 & 5.264 & 3.637 & 3.048 & 2.524 & 2.281 & 2.140 \\
40 & 32.109 & 25.541 & 18.967 & 12.372 & 9.052 & 5.673 & 3.894 & 3.255 & 2.681 & 2.406 & 2.249 \\
50 & 34.203 & 27.194 & 20.177 & 13.140 & 9.596 & 5.991 & 4.095 & 3.414 & 2.808 & 2.508 & 2.333 \\
60 & 35.918 & 28.547 & 21.168 & 13.768 & 10.042 & 6.252 & 4.260 & 3.543 & 2.913 & 2.595 & 2.405 \\
70 & 37.370 & 29.693 & 22.007 & 14.299 & 10.419 & 6.472 & 4.399 & 3.652 & 3.002 & 2.672 & 2.469 \\
80 & 38.628 & 30.686 & 22.735 & 14.760 & 10.746 & 6.664 & 4.520 & 3.747 & 3.077 & 2.740 & 2.527 \\
90 & 39.740 & 31.563 & 23.377 & 15.167 & 11.035 & 6.833 & 4.627 & 3.831 & 3.143 & 2.801 & 2.580 \\
100 & 40.735 & 32.348 & 23.952 & 15.532 & 11.294 & 6.984 & 4.722 & 3.907 & 3.201 & 2.855 & 2.629 \\
\hline
\end{tabular}

Table 8.2: The expected number of queries $b_{c}(n)$ in the $c$-bisection auction.

\begin{tabular}{r|rrrrrrrrrrr}
$n \backslash c$ & $1 / 10$ & $1 / 8$ & $1 / 6$ & $1 / 4$ & $1 / 3$ & $1 / 2$ & $2 / 3$ & $3 / 4$ & $5 / 6$ & $7 / 8$ & $9 / 10$ \\
\hline 2 & 11.5 & 9.5 & 7.5 & 5.4 & 4.4 & 3.3 & 2.8 & 2.5 & 2.3 & 2.3 & 2.2 \\
3 & 21.8 & 17.8 & 13.8 & 9.7 & 7.7 & 5.6 & 4.4 & 4.0 & 3.7 & 3.5 & 3.4 \\
4 & 32.0 & 26.0 & 20.0 & 13.9 & 10.9 & 7.8 & 6.1 & 5.5 & 5.0 & 4.7 & 4.6 \\
5 & 42.2 & 34.2 & 26.2 & 18.1 & 14.0 & 9.9 & 7.7 & 6.9 & 6.3 & 6.0 & 5.8 \\
6 & 52.4 & 42.4 & 32.3 & 22.3 & 17.2 & 12.0 & 9.3 & 8.4 & 7.6 & 7.2 & 7.0 \\
7 & 62.5 & 50.5 & 38.5 & 26.4 & 20.3 & 14.1 & 10.9 & 9.8 & 8.8 & 8.4 & 8.1 \\
8 & 72.6 & 58.6 & 44.6 & 30.5 & 23.4 & 16.2 & 12.5 & 11.2 & 10.1 & 9.6 & 9.3 \\
9 & 82.7 & 66.7 & 50.7 & 34.9 & 26.5 & 18.3 & 14.1 & 12.6 & 11.3 & 10.8 & 10.4 \\
10 & 92.8 & 74.8 & 56.8 & 38.7 & 29.6 & 20.4 & 15.6 & 14.0 & 12.6 & 12.0 & 11.6 \\
20 & 193.5 & 155.4 & 117.4 & 79.3 & 60.1 & 40.9 & 31.0 & 27.7 & 24.9 & 23.7 & 23.0 \\
30 & 293.8 & 235.8 & 177.7 & 119.6 & 90.4 & 61.1 & 46.3 & 41.2 & 37.1 & 35.3 & 34.3 \\
40 & 394.1 & 316.1 & 238.0 & 159.8 & 120.7 & 81.3 & 61.4 & 54.7 & 49.2 & 46.8 & 45.5 \\
50 & 494.3 & 396.3 & 298.2 & 200.1 & 150.9 & 101.5 & 76.6 & 68.1 & 61.3 & 58.3 & 56.7 \\
60 & 594.5 & 476.4 & 358.4 & 240.2 & 181.0 & 121.6 & 91.7 & 81.6 & 73.4 & 69.8 & 67.9 \\
70 & 694.6 & 556.6 & 418.5 & 280.3 & 211.1 & 141.7 & 106.8 & 95.0 & 85.5 & 81.3 & 79.0 \\
80 & 794.8 & 636.7 & 478.6 & 320.4 & 241.2 & 161.8 & 121.9 & 108.4 & 97.5 & 92.8 & 90.2 \\
90 & 894.9 & 716.8 & 538.7 & 360.5 & 271.4 & 181.9 & 136.9 & 121.8 & 109.6 & 104.3 & 101.3 \\
100 & 994.9 & 796.9 & 598.8 & 400.6 & 301.4 & 202.0 & 152.0 & 135.2 & 121.6 & 115.8 & 112.5 \\
\hline
\end{tabular}




\section{Bibliography}

[1] L. Ausubel and P. Milgrom. Ascending auctions with package bidding. Frontiers of Theoretical Economics, 1, 2002.

[2] L. Ausubel and P. Milgrom. The lovely but lonely Vickrey auction. In P. Cramton, Y. Shoham, and R. Steinberg, editors, Combinatorial Auctions. MIT Press, 2006.

[3] D. Bergmann and J. Välimäki. Information acquisition and efficient mechanism design. Econometrica, 70:1007-1034, 2002.

[4] L. Blumrosen and N. Nisan. Auctions with severely bounded communication. In Proccedings of 43th Annual Symposium on Foundations of Computer Science (FOCS 02), pages 406-416, Vancouver, Canada, 2002.

[5] L. Blumrosen, N. Nisan, and I. Segal. Multi-player and multi-round auctions with severely bounded communication. In Proccedings of 11th Annual European Symposium on Algorithms (ESA 03), Budapest, Hungary, 2003.

[6] F. Brandt. Fundamental Aspects of Privacy and Deception in Electronic Auctions. PhD thesis, Department for Computer Science, Technical University of Munich, 2003.

[7] M. S. Y. Chwe. The discrete bid first auction. Economics Letters, 31:303-306, 1989.

[8] E. H. Clarke. Multipart pricing of public goods. Public Choice, XI:17-33, 1971.

[9] O. Compte and P. Jehiel. On the virtues of the ascending price auction: New insights in the private value setting. Technical report, 2000. 
[10] O. Compte and P. Jehiel. Auction and information acquisition: Sealed-bid or dynamic formats? Working paper, 2001.

[11] W. Conen and T. Sandholm. Preference elicitation in combinatorial auctions: Extended abstract. In Proceedings of the ACM Conference on Electronic Commerce (ACM-EC), pages 256-259, Melbourne, Australia, 2001.

[12] W. Conen and T. Sandholm. Partial-revelation VCG mechanism for combinatorial auctions. In Proceedings of Eighteenth National Conference on Artificial Intelligence (AAAI), pages 367-372, 2002.

[13] V. Conitzer and T. Sandholm. Computational criticisms of the revelation principle. In Proceedings of the AAMAS-03 Workshop on Agent Mediated Electronic Commerce V (AMEC V), Melbourne, Australia, 2003.

[14] V. M. Coppinger, V. L. Smith, and J. A. Titus. Incentives and behavior in English, Dutch and sealed-bid auctions. Economic Inquiry, 43:1-22, 1980.

[15] J. C. Cox, B. Roberson, and V. L. Smith. Theory and behavior of single object auctions. In V. L. Smith, editor, Combinatorial Auctions, volume 2, Greenwich, Conn., 1982. JAI Press.

[16] E. David, A. Rogers, J. Schiff, S. Kraus, and N. R. Jennings. Optimal design of English auctions with discrete bid levels. In Proceedings of ACM Conference on Electronic Commerce (EC'05), pages 98-107, Vancouver, Canada, 2005.

[17] R. Engelbrecht-Wiggans and C. M. Kahn. Protecting the winner: Second-price versus oral auctions. Economics Letters, 35(3):243-248, 1991.

[18] U. Fischbacher. Z-Tree: Zurich toolbox for redymade economic experiments. Working paper 21, Institute for Empirical Research in Economics, University of Zurich, 1999.

[19] Y. Fujishima, D. McAdams, and Y. Shoham. Speeding up ascending-bid auctions. In Proceedings of the Sixteenth International Joint Conference on Artificial Intelligence, pages 554-563, 1999.

[20] J. Green and J.-J. Laffont. Characterization of satisfactory mechanisms for the revelation of preferences for public goods. Econometrica, 45:427-438, 1977.

[21] E. Grigorieva, P. J.-J. Herings, R. Müller, and D. Vermeulen. The communication complexity of private value single item auctions. Operations Research Letters, 34(5):491-498, 2006. 
[22] E. Grigorieva, P. J.-J. Herings, R. Müller, and D. Vermeulen. The family of c-bisection auctions: efficiency and running time. METEOR Research Memorandum RM/06/019, Maastricht University, 2006.

[23] E. Grigorieva, P. J.-J. Herings, R. Müller, and D. Vermeulen. Inefficiency of equilibria in query auctions with continuous valuations. METEOR Research Memorandum RM/06/017, Maastricht University, 2006.

[24] E. Grigorieva, P. J.-J. Herings, R. Müller, and D. Vermeulen. The private value single item bisection auction. Economic Theory, 30:107-118, 2007.

[25] E. Grigorieva and M. Strobel. Bidding behavior in the bisection auction an experimental investigation. METEOR Research Memorandum RM/06/26, Maastricht University, 2006.

[26] T. Groves. Incentives in teams. Econometrica, 61:617-631, 1973.

[27] R. M. Harstad. Dominant strategy adoption and bidder's experience with pricing rules. Experimental Economics, 3:261-280, 2000.

[28] B. Holmström. Groves schemes on restricted domains. Econometrica, 47:11371144, 1979 .

[29] J. H. Kagel. Auctions: A survey of experimental research. In J. Kagel and A. Roth, editors, The handbook of experimental economics. New Jersey: Princeton University Press, 1995.

[30] J. H. Kagel, R. M. Harstad, and D. Levin. Information impact and allocation rules in auctions with affiliated values: A laboratory study. Econometrica, 55(6):1275-1304, 1987.

[31] J. H. Kagel and D. Levin. Independent private value auctions: bidder behaviour in first-, second- and third-price auctions with varying numbers of bidders. The Economic Journal, 103:868-879, 1993.

[32] D. Knuth. The Art of Computer Programming; Volume 1: Fundamental Algorithms. Massachusetts: Addison-Wesley, 1997.

[33] A. Kress and C. Boutilier. A study of limited-precision, incremental elicitation in auctions. In Proceedings of the 4th International Conference on Autonomous Agents and Multiagent Systems, 2004.

[34] V. Krishna. Auction Theory. Academic Press, 2002. 
[35] E. Kushilevitz and N. Nisan. Communication complexity. Cambridge University Press, 1996.

[36] K. Larson and T. Sandholm. Computationally limited agents in auctions. In Proceedings of the International Conference on Autonomous Agents, pages 2734, Montreal, Canada, 2001.

[37] K. Larson and T. Sandholm. Costly valuation computation in auctions. In Proceedings of the Theoretical Aspects of Reasoning about Knowledge (TARK), pages 169-182, Siena, Italy, 2001.

[38] H. Levy. Stochastic dominance. Kluwer Academic Publishers, Norwell, MA, 1998.

[39] D. Lucking-Reiley. Auctions on the internet: What's being auctioned, and how? Journal of Industrial Economics, 48(3):227-252, 2000.

[40] D. Lucking-Reiley. Vickrey auctions in practice: From nineteenth-century philately to twenty-first-century e-commerce. Journal of Economic Perspectives, 14(3):183-192, 2000.

[41] A. Mas-Colell, M. Whinston, and J. Green. Microeconomic Theory. New York, Oxford University Press, 1995.

[42] P. R. Milgrom and R. J. Weber. A theory of auctions and competitive bidding. Econometrica, 50:1089-1122, 1982.

[43] B. Moldovanu and P. Jehiel. The European UMTS/IMT-2000 licence auctions. CERP Discussion Paper, 2810, 2001.

[44] M. Naor, B. Pinkas, and R. Sumner. Privacy preserving auctions and mechanism design. In Proceedings of ACM Conference on Electronic Commerce, 1999 .

[45] N. Nisan and I. Segal. The communication requirements of efficient allocations and supporting prices. Journal of Economic Theory, 2005. to appear.

[46] M. J. Osborne and A. Rubinstein. A Course in Game Theory. MIT Press, 1994.

[47] D. Parkes. ibundle: An efficient ascending price bundle auction. In Proccedings of 1st ACM Conference on Electronic Commerce, pages 148-157, 1999. 
[48] D. Parkes. Optimal auction design for agents with hard valuation problems. In Proccedings of IJCAI-99 Workshop on Agent Mediated Electronic Commerce, pages 206-219, Stockholm, Sweden, 1999.

[49] D. Parkes. Iterative combinatorial auctions: Achieving economic and computational efficiency. PhD thesis, University of Pennsylvania, 2001.

[50] D. Parkes. Auction design with costly preference elicitation. Annals of Mathematics and AI (Special Issue on the Foundations of Electronic Commerce), pages 269-302, 2005.

[51] M. H. Rothkopf and R. M. Harstad. On the role of discrete bid levels in oral auctions. European Journal of Operational Research, 74:572-581, 1994.

[52] M. H. Rothkopf, T. J. Tisberg, and E. P. Kahn. Why are Vickrey auctions rare? Journal of Political Economy, 98:94-109, 1990.

[53] A. Rubinstein. Modeling Bounded Rationality. MIT Press, 1998.

[54] T. Sandholm. Issues in computational Vickrey auctions. International Journal of Electronic Commerce, 4.

[55] S. Siegel and N. J. Castellan. Nonparametric statistics for the behavioral sciences. McGraw-Hill Book Company, 1988.

[56] H. A. Simon. A behavorial model of rational choice. Quarterly Journal of Economics, 69:99-118, 1955.

[57] V. L. Smith. Relevance of laboratory experiments to testing resource allocation theory. In J. Kinsata and J. Ramsey, editors, Evaluation of econometric models, pages 345-377. Academic Press, New York, 1980.

[58] E. Van Damme. The European UMTC auctions. Europian Economic Review, $46: 846-858,2002$.

[59] W. Vickrey. Counterspeculation, auctions and competitive sealed tenders. Journal of Finance, 16:8-37, 1961.

[60] P. Wurman and M. Wellman. AkBA: A progressive anonymous-price combintorial auction. In Proccedings of ACM Conference on Electronic Commerce, pages 21-29. Minneapolis, MN, 2000.

[61] B. S. Yamey. Why 2,310,000 [pounds] for a Velazqueez?: An auction bidding rule. Journal of Political Economy, 80:1323-1327, 1972. 
[62] J. Yu. Discrete approximation of continous allocation mechanisms. PhD thesis, California Institute of Thechnology, Division of Humanities and Social Science, 1999. 


\section{Subject Index}

algorithm, 49

acyclic, 54

bisection, 51

cyclic, 54

optimal, 54

preferred, 53

Vickrey, 50

auction, 11

c-bisection, 122

bisection, 24

combinatorial, 13

direct, 102

direct $c$-bisection, 132

Dutch, 12

English, 12, 21, 23, 100

ex post individually rational, 102

general bisection, 107, 121

query, 102

regular general bisection, 116

sealed bid first price, 12

sealed bid second price, 12,21

Vickrey, 12, 21, 102

behavioral equivalence, 60

best response, 102

binary tree, 50

characteristic function, 53 communication

measure of, 38

data transmission, 38

double, 55

efficiency

absolute loss of, 71

allocative, 13, 71

relative loss of, 71

equilibrium

efficient, 22

ex post, 101

Nash, 101

query, 105

sometimes finite, 105

ex post game, 101

extensive form game, 26, 109

first-order stochastic dominance, 54

information

bit, 38

revelation, $14,22,37$

set, 27

overbidding, 64

performance measure, 13 
Revelation Principle, 14

severe deviation, 68

single, 55

strategic equivalence, 22,60

strategy, 29, 101

bluff, 113, 123

dominant, 102

ex post individually rational, 102

threshold, 29

truth-telling, 22, 34

weakly dominant, 22

strategy profile, 101

ex post efficient, 102

threshold, 30

maximum, 32

second-highest, 32

treatment

BA, 62

EA, 62

VA, 62

underbidding, 64

value bid, 63

VCG mechanism, 14, 48

Vickrey outcome, 48

welfare, 137

loss of, 137

maximization of, 13

worst-case analysis, 52 


\section{Nederlandse samenvatting}

De efficinte verdeling van goederen over economische agenten is een van de centrale thema's in de economische theorie. Een van de meest gebruikte manieren om goederen te verdelen is het markt mechanisme. En een van de bekendste vormen van een markt mechanisme is het veiling mechanisme. Traditiegetrouw worden met name verzamelobjecten, kunstvoorwerpen, en antiek via veilingen verkocht. Maar gedurende de laatste jaren zijn zowel de variteit in goederen alsmede het totale volume sterk toegenomen als gevolg van de toepassing van moderne communicatie en informatie systemen om veilingen te organiseren. En door de toegenomen populariteit van veilingen wordt er momenteel in de economische wetenschap druk gezocht naar nieuwe en adequate ontwerpen voor veilingen.

Er zijn verschillende manieren om te vergelijken hoe adequaat verschillende ontwerpen voor een veiling nu eigenlijk zijn. Pareto efficintie van de resulterende allocatie is een van de criteria om die vergelijking te maken. Dit criterium is zeker van belang als een publiek goed wordt uitbesteed aan de private sector. Veel onderzoek heeft zich gericht op het ontwerp van veilingen die, ook als bieders uitsluitend op basis van eigenbelang handelen, toch een efficinte allocatie garanderen. Het merendeel van dit onderzoek heeft zich onder invloed van het zogeheten Revelation Principle vooral gericht op zogeheten direct revelation mechanisms. Algemeen wordt aangenomen dat in de private value setting het allocatie probleem is opgelost, omdat in het VCG mechanisme-een speciaal type direct revelation mechanismwaarheidsgetrouw rapporteren een zwak dominante strategie is en het evenwicht dientengevolge resulteert in een efficinte allocatie.

Echter, in het VCG mechanisme eist de implementatie van de evenwichtsstrategie dat bieders hun preferenties volledig en exact rapporteren. En er zijn verschillende redenen waarom bieders niet altijd bereid zijn dit ook inderdaad te doen. Ten eerste kunnen bieders besluiten niet alle informatie omtrent hun preferenties 
te rapporteren, om op die manier hun concurrentiepositie op de markt te kunnen handhaven. Ten tweede kan een exacte bepaling van preferenties veel rekencapaciteit vergen. Tenslotte kan een volledige rapportage van preferenties een onacceptabele hoeveelheid communicatie met zich mee brengen.

Erkenning dat volledige rapportage van preferenties niet altijd gewenst is heeft geleid tot een hernieuwde belanstelling voor veilingen waar bieders niet noodzakelijkerwijze verplicht worden al hun informatie prijs te geven. De uitdaging, die ook in dit proefschrift wordt aangegaan, is dus om veilingen te ontwerpen die een efficinte, of zo efficint mogelijke, allocatie bewerkstelligen-bij voorkeur via een evenwicht in zwak dominante strategien-zonder bieders te verplichten volledige en exacte informatie omtrent preferenties te geven.

In dit proefschrift wordt dus onderzocht hoe de vraag naar informatie beperkt kan worden zonder de efficintie van de allocatie aan te tasten. We beschrijven een nieuw type iteratieve veiling, de bisectie veiling, en tonen aan dat, als de waarderingen van de bieders voor het te veilen object geheeltallig zijn, de allocatie in deze veiling inderdaad efficint is, terwijl de benodigde hoeveelheid informatie om deze allocatie te bewerkstelligen veel lager is dan in alle reeds bekende efficinte veilingen. Voor de setting met geheeltallige waarderingen ontwerpen we een iteratieve implementatie van het VCG mechanisme die voor het bereiken van een efficinte allocatie slechts een minimale hoeveelheid informatie van de bieders vraagt. In de setting waar waarderingen continue waarden kunnen aannemen laten we zien dat een beperking van de gevraagde hoeveelheid informatie noodzakelijkerwijze inefficinties in de allocatie met zich meebrengt. We berekenen in het bijzonder de minimale hoeveelheid informatie die nodig is om een bepaald niveau van efficintie te bereiken.

Het eerste deel van het proefschrift behandelt de setting waar waarderingen van bieders geheeltallig zijn. We beschrijven en analyseren de bisectie veiling. In Hoofdstuk 2 tonen we aan dat deze veiling strategisch equivalent is met de Vickrey veiling en de Engelse veiling. Dientengevolge is ook in deze veiling waarheidsgetrouw rapporteren een zwak dominante strategie, en is de allocatie efficint in het evenwicht.

Hoewel de bisectie veiling strategisch equivalent is met de Vickrey veiling en de Engelse veiling, heeft de bisectie veiling minder informatie en communicatie nodig om de allocatie te bepalen dan de andere twee veilingen. Dit wordt aangetoond in Hoofdstuk 3, waar wordt berekend wat voor elk van deze drie veilingen precies de gemiddelde hoeveelheid informatie is die in het evenwicht gerapporteerd moet worden. In Hoofdstuk 4 vergelijken we hoeveel communicatie een efficinte veiling minimaal nodig heeft om een allocatie te bepalen. Om deze vergelijkingen te maken hanteren we een nieuw criterium voor de performance van een algoritme. 
Dit criterium is gebaseerd op het begrip stochastische dominantie. We laten zien dat volgens dit criterium, in de setting met 2 spelers, de bisectie veiling minder communicatie nodig heeft dan willekeurig welke andere efficinte veiling.

Gegeven de bovengenoemde voordelen van de bisectie veiling ligt het voor de hand om te kijken hoe goed deze veiling in de praktijk werkt. Daarom is de performance van de bisectie veiling ook in een laboratorium experiment getest. In het experiment vergelijken we het biedgedrag van de bieders, alsmede de efficintie van de resulterende allocatie, voor zowel de bisectie veiling als voor de Vickrey en de Engelse veiling. We kijken in het bijzonder of bieders de dominante strategie volgen en waarheidsgetrouw rapporteren. Verder bekijken we of het biedgedrag verandert als bieders meer ervaring hebben met het bieden in deze veilingen. De data tonen aan dat de bisectie veiling betere resultaten geeft dan de Vickrey veiling. De Engelse veiling is alleen volgens enkele specifieke criteria beter dan de bisectie veiling. Hoofdstuk 5 beschrijft het experiment en presenteert de resultaten die we in dit experiment vonden.

In het tweede deel van dit proefschrift bestuderen we de performance van zogeheten query veilingen in een setting waar waarderingen van bieders een continuum van waarden aan kunnen nemen. In Hoofdstuk 6 laten we zien dat query veilingen weliswaar een bescheiden hoeveelheid informatie voor allocatie vergen, maar praktisch gesproken nooit efficint kunnen zijn. We bewijzen dat volledige efficintie alleen kan worden bereikt als we accepteren dat de looptijd van de veiling oneindig is voor bijna alle realisaties van de waarderingen. Dit roept de vraag op wat de prijs is in termen van looptijd van de veiling om een bepaald niveau van efficintie te kunnen garanderen. De laatste twee hoofdstukken van dit proefschrift geven een antwoord op deze vraag. In Hoofdstuk 7 introduceren we een algemene klasse van query veilingen, gegeneraliseerde bisectie veilingen genaamd. Binnen deze klasse tonen we de existentie van een specifiek type ex post evenwichten-zogeheten bluf evenwichten-aan, en laten zien dat deze evenwichten onvermijdelijk inefficint zijn. In Hoofdstuk 8 gaan we voor een speciaal type gegeneraliseerde bisectie veilingen, de $c$-bisectie veilingen, nader in op de trade-off tussen looptijd en efficintie in het bluf evenwicht. We tonen aan dat door een juiste keuze van de parameter $c$ de inefficintie in het bluf evenwicht willekeurig klein kan worden gemaakt, terwijl de looptijd van de veiling eindig is voor alle realisaties van de waarderingen van de bieders. 



\section{Curriculum Vitae}

Elena Grigorieva was born on August 23, 1973 in Chita, Russia. In June of 1990, she received her high school diploma from Novosibirsk Physics and Mathematics School. In September of 1990, she started studying Quantitative Economics at Novosibirsk State University, and in June of 1995, she was awarded her Master's Degree with distinction (cum laude) in "Mathematical Models in Economics". From 1995 until 1999, Elena was employed as a business analyst by the Centre of Financial Technologies and Promstroybank of Russia. In 2000, she moved to the Netherlands where she joined the E-commerce research group at the International Institute of Infonomics, Heerlen. A year later, she started her PhD research at the Faculty of Economics and Business Administration at Maastricht University. The results of her research are presented in this thesis. 